\title{
MÉMOIRE DE MAÎTRISE
}

PRÉSENTÉ À

L'UNIVERSITÉ DU QUÉBEC À CHICOUTIMI

COMME EXIGENCE PARTIELLE

DE LA MAÎTRISE EN INFORMATIQUE

\section{PAR}

Jean-Sébastien Bilodeau

B.Sc.A.

Algorithme de localisation par multilatération d'une puce RFID passive pour la reconnaissance d'activités dans un habitat intelligent 


\section{RÉSUMÉ}

Le vieillissement de la population provoque une forte croissance du nombre de personnes atteintes de troubles cognitifs, telle que ceux découlant de la maladie d'Alzheimer, qui causent (chez les patients) un comportement incohérent qui limite leur capacité à réaliser leurs tâches de la vie courante. Ces personnes souhaitent demeurer le plus longtemps possible à domicile et jouir pleinement de leur autonomie, même si celle-ci est en déclin. Pour ce faire, l'environnement physique et humain des résidences doit être adapté, voir augmenté à l'aide de la technologie, afin de répondre aux besoins, de pallier aux incapacités cognitives et physiques, d'assurer la sécurité et de supporter les résidents dans leurs activités quotidiennes. C'est pourquoi, depuis quelques années, une communauté croissante de chercheurs tente de trouver des réponses technologiques à cette problématique complexe grâce au concept d'habitat intelligent. Cette approche propose d'exploiter des capteurs ubiquitaires dissimulés dans l'environnement pour surveiller et détecter les anomalies comportementales liées aux déficits cognitifs pour ensuite effectuer une guidance appropriée (des rappels) sous forme de messages vocaux, lumineux ou vidéo. Ainsi, cette assistance technologique pourrait apporter une solution abordable et fournir une alternative viable dans le but de reporter l'institutionnalisation. Par ailleurs, la principale problématique liée à l'implémentation d'un tel habitat visant le soutien des aînés concerne la reconnaissance des activités du résident. Cette problématique est primordiale, car le soutien technologique à domicile ne peut se faire que si le système arrive à interpréter correctement le comportement du patient et à détecter ses erreurs. Bien qu'un grand nombre d'informations puissent être exploitées par les algorithmes de reconnaissance, il est clair que la position en temps réel des objets et du patient dans l'environnement constitue la pierre angulaire d'un système efficace. Dans cette optique, la technologie RFID passive, qui permet de localiser et d'identifier les objets qui sont manipulés dans l'habitat, s'est imposée comme dominante dans le secteur. Cette technologie, peu coûteuse, offre une grande robustesse (ex. les objets étiquetés peuvent aller au lave-vaisselle), en plus de ne nécessiter aucun support énergétique (pas de piles). Cependant, sa grande faiblesse est d'offrir peu de précision quant à la position réelle des objets dans l'environnement. Pourtant, cette précision est essentielle si l'on souhaite reconnaître avec justesse les activités et les anomalies.

Afin de trouver des solutions à la problématique que nous venons de citer, ce mémoire propose de formaliser et d'évaluer un nouvel algorithme novateur de localisation d'étiquettes RFID passive visant une utilisation dans un contexte de reconnaissance d'activités et d'erreurs cognitives. Par conséquent, la contribution à caractère théorique du mémoire est de proposer un algorithme amélioré de positionnement par multilatération à partir de plusieurs ellipses, augmenté de plusieurs filtres réduisant l'imprécision. Par la suite, la contribution pratique et expérimentale du mémoire consiste en une implémentation de ce modèle algorithmique à travers un système complet de localisation, qui a été déployé et testé dans un environnement réel d'habitat intelligent équipé d'une infrastructure RFID. Les résultats obtenus sont comparés à ceux de la littérature et montrent bien l'avantage de l'approche. Enfin, nous montrerons comment nous avons appliqué ce nouvel algorithme de positionnement à l'intérieur d'un système de reconnaissance d'activités, montrant ainsi l'utilité effective du modèle dans ce contexte précis. 


\section{REMERCIEMENTS}

En préambule à ce mémoire, je souhaite adresser tous mes remerciements aux personnes qui m'ont apporté leur aide et qui ont contribué à l'élaboration de ce mémoire.

Tout d'abord, je désire remercier mon directeur de recherche Bruno Bouchard et mes codirecteurs Sébastien Gaboury et Abdenour Bouzouane pour leur implication dans le projet, leur dévouement, leurs encouragements et leurs conseils.

Ensuite, je tiens à signaler que le projet de recherche présenté dans ce mémoire fait partie des activités du laboratoire d'intelligence ambiante pour la reconnaissance d'activités (LIARA) à l'université du Québec à Chicoutimi (UQAC). Je tiens également à remercier un collègue au LIARA, Dany Fortin Simard, pour sa collaboration dans ce projet.

Finalement, je tiens à remercier mes parents pour leur soutien inconditionnel tout au long de ce projet. J'adresse un merci tout particulier à ma mère qui s'est tenue régulièrement informée de l'avancée de mes différents travaux. 


\section{TABLE DES MATIÈRES}

RÉSUMÉ

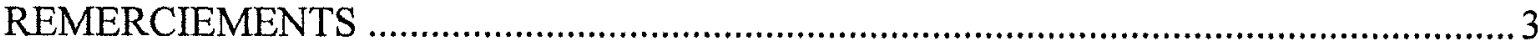

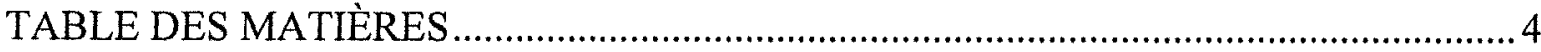

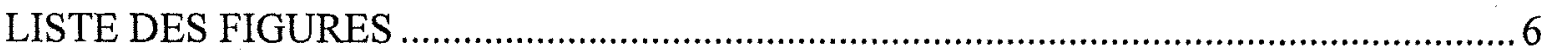

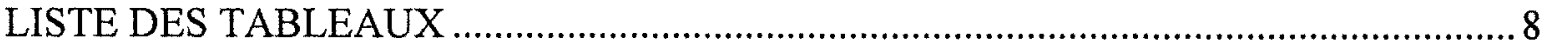

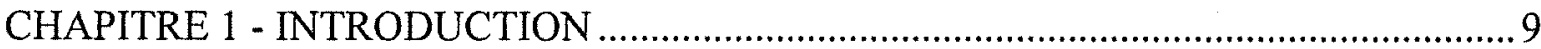

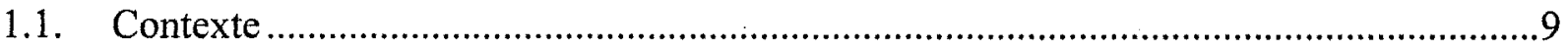

1.2. Problématique ciblée dans ce mémoire...........................................................................11

1.3. Travaux antérieurs liés au positionnement par RFID ...................................................13

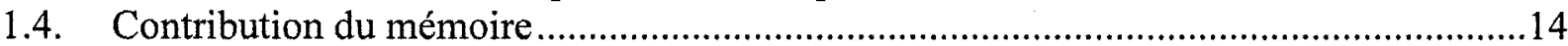

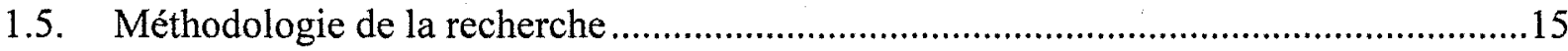

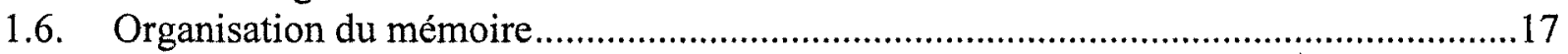

\section{CHAPITRE 2 - APPROCHES DE LOCALISATION EN ENVIRONNEMENT INTÉRIEUR...20}

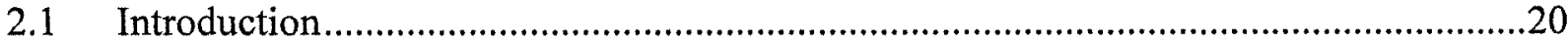

2.2 Identification par radio fréquence (RFID - Radio-Frequency Identification) .................21

2.3 Évaluation des impacts des RSS sur un système de localisation ....................................25

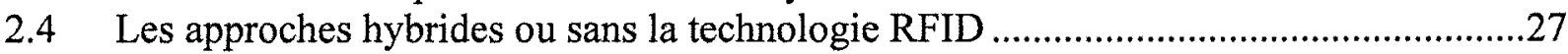

2.4.1 L'utilisation des ultrasons ............................................................................................27

2.4.2 La combinaison de la vision ou d'un laser avec la technologie RFID .................................28

2.4.3 La combinaison de la technologie RFID avec un capteur de mouvement..........................28

2.4.4 Bilan sur les approches hybrides.................................................................................29

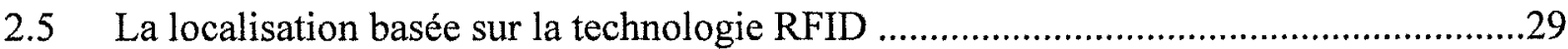

2.6 Les approches basées sur la trilatération......................................................................32

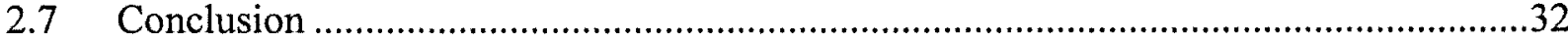

CHAPITRE 3 - MULTILATÉRATION AMÉLIORÉE ..................................................34

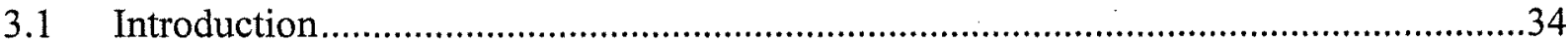

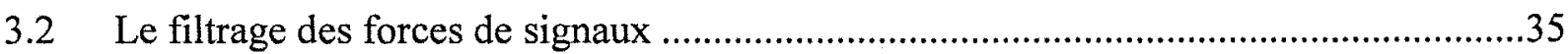

3.2.1 Filtre de réduction des faux positifs et des faux négatifs .................................................35

3.2.2 Filtre de réduction de crête ou de variation anormalement élevée ....................................37

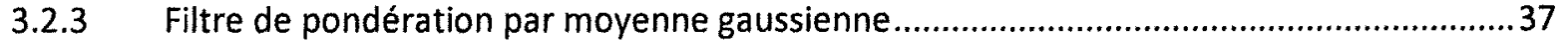


3.3 La multilatération elliptique

3.3.1 Multilatération par intersection géométrique de disques elliptiques.

3.3.2 Recherche ou raffinement de la solution ...................................................................42

3.4 Règles définissant le comportement du mouvement des objets.................................42

3.4.1 La position, la vitesse et le mouvement......................................................................43

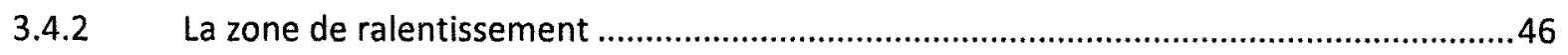

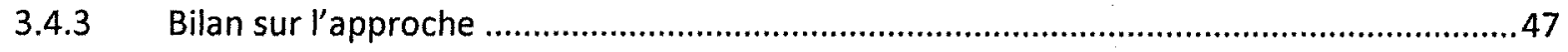

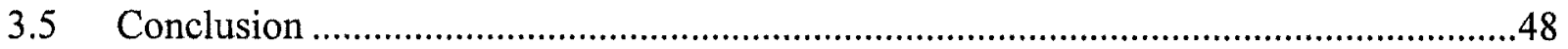

CHAPITRE 4 - IMPLÉMENTATION ET VALIDATIONS..........................................50

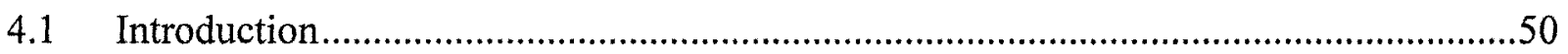

4.2 Implémentation dans le laboratoire du LIARA ...................................................50

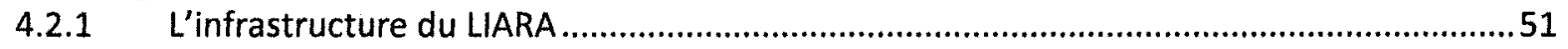

4.2.2 Implémentation du système de positionnement .........................................................54

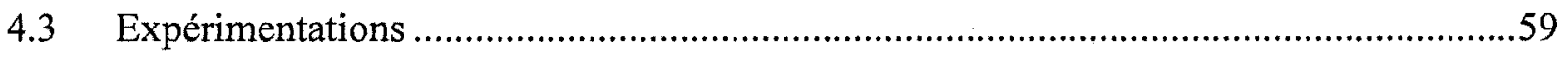

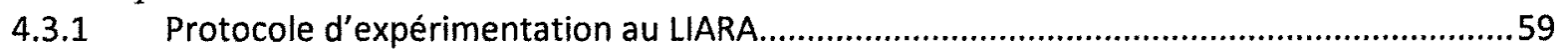

4.3.2 Expérimentation avec le système de reconnaissance de comportements erronés ...........64

4.3.3 Contribution (transfert technologique) à l'industrie (TLM) ............................................66

4.4 Analyse et comparaison avec les résultats présentés dans les ouvrages connexes.........71

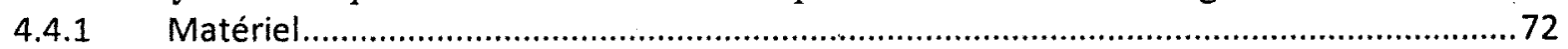

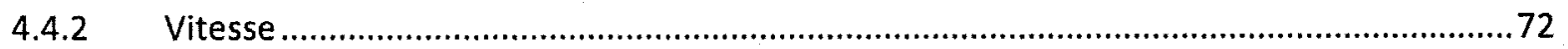

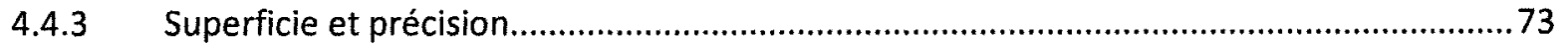

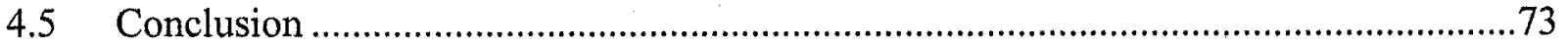

CHAPITRE 5 - CONCLUSION GÉNÉRALE …....................................................... 74

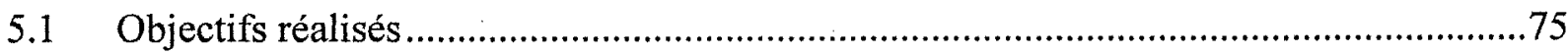

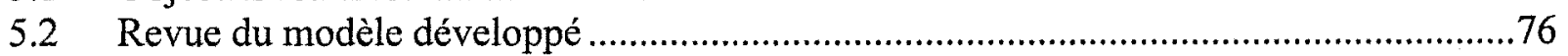

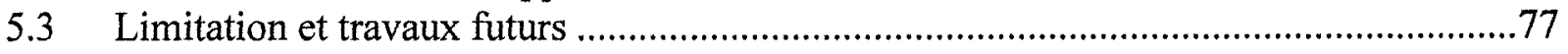

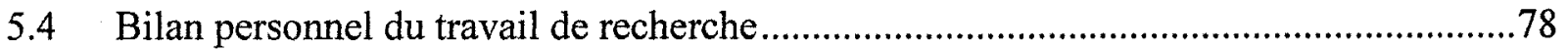

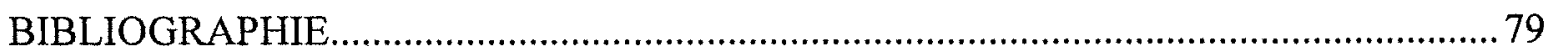




\section{LISTE DES FIGURES}

FIGURE 1.1 : SYSTÈME RFID - ANTENNES, MODULE DE COLLECTE ET ÉTIQUETTES (ADAPTÉ DE [19]).

FIGURE 2.1 : INTERSECTION DE 3 CERCLES (ADAPTÉ DE [40]) ..........................................................2 23

FIGURE 2.2 : K-PLUS PROCHES VOISINS (ADAPTÉ DE [16]) ….............................................................2 24

FIGURE 2.3 : CORRÉLATION DES RSSI EN FONCTION DES DISTANCES [41] ….......................................25

FIGURE 2.4 : IMPRÉCISION DES RSSI EN FONCTION DE LA DISTANCE [41] ............................................26

FIGURE 3.1 : TRAITEMENT EN TEMPS RÉEL DE LA FORCE DU SIGNAL D'UNE ÉTIQUETTE RFID STATIQUE. LA FORCE DU SIGNAL BRUTE EST EN BLEU ET LA FORCE DU SIGNAL FILTRÉ EST

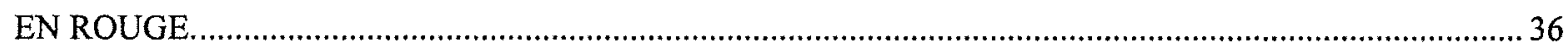

FIGURE 3.2 : TRAITEMENT EN TEMPS RÉEL DE LA FORCE DU SIGNAL D'UNE ÉTIQUETTE RFID STATIQUE. LA FORCE DU SIGNAL BRUTE EST EN BLEU ET LA FORCE DU SIGNAL FILTRÉ EST EN ROUGE.

FIGURE 3.3 : TRAITEMENT EN TEMPS RÉEL DE LA FORCE DU SIGNAL D'UNE ÉTIQUETTE RFID STATIQUE. LA FORCE DU SIGNAL BRUTE EST EN BLEU ET LA FORCE DU SIGNAL FILTRÉ EST EN ROUGE......

FIGURE 3.4 : POSITIONNEMENT EN TEMPS RÉEL D'UN OBJET. LES ELLIPSES VERTES SONT CALCULÉES À PARTIR DES RSSI, LE CARRÉ VERT EST LA POSITION CALCULÉE PAR L'ALGORITHME ET LE CARRÉ ROUGE EST LA POSITION RÉELLE DE L'OBJET. 41

FIGURE 3.5 : POSITIONNEMENT EN TEMPS RÉEL D'UN OBJET. LES ELLIPSES VERTES SONT CALCULÉES À PARTIR DES RSSI ET LES ELLIPSES EN POINTILLÉES SONT CELLES CORRIGÉES PAR L'ALGORITHME DE POSITIONNEMENT. LE CARRÉ VERT EST LA POSITION ESTIMÉE PAR L'ALGORITHME DE POSITIONNEMENT TANDIS QUE LE CARRÉ ROUGE EST LA POSITION

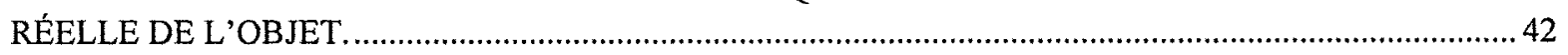

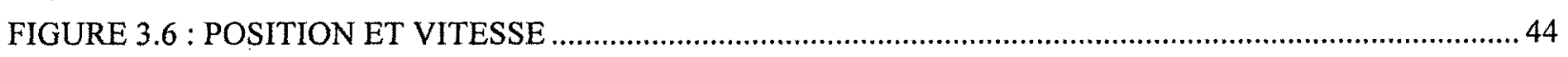

FIGURE 3.7 : FORCES RÉGISSANT LE COMPORTEMENT DE MOUVEMENT .............................................. 45

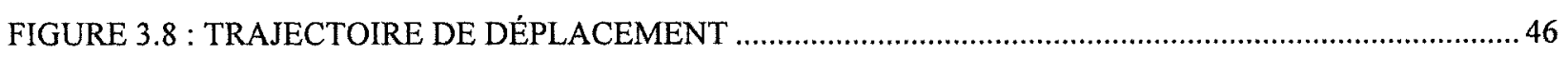

FIGURE 3.9 : COMPORTEMENT D'ARRIVÉ VERS LA CIBLE ..................................................................... 46

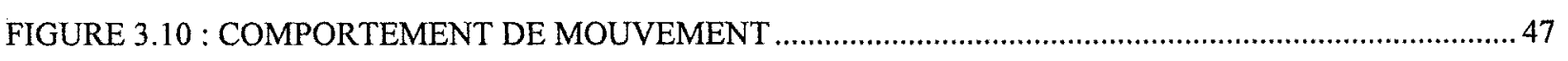

FIGURE 3.11 : DÉROULEMENT DU PROCESSUS DE LOCALISATION .......................................................... 48

FIGURE 4.1 : IMAGES DE L'HABITAT INTELLIGENT DU LIARA ................................................................51

FIGURE 4.2 : OBJETS MUNIS D'ÉTIQUETTES RFID PASSIVES..................................................................5 54

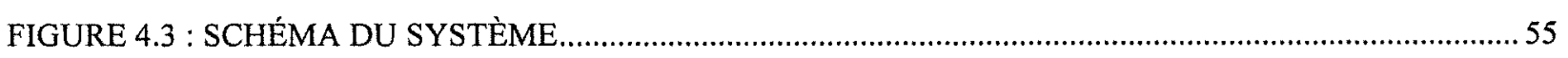

FIGURE $4.4:$ INTERFACE UTILISATEUR AVEC LA VUE 2D ........................................................................56

FIGURE 4.5 : INTERFACE UTILISATEUR AVEC LA VUE 3D ....................................................................5

FIGURE 4.6 : AMÉLIORATION DE LA PRÉCISION D'UN OBJET STATIQUE AVEC L'OUTIL DE CALIBRATION. 
FIGURE 4.7 : AMÉLIORATION DE LA PRÉCISION D'UN OBJET DYNAMIQUE AVEC L'OUTIL DE CALIBRATION.

FIGURE 4.8 : PRÉCISION SOUS DIFFÉRENTES CONFIGURATIONS

FIGURE 4.9 : RÉPARTITION DE L'IMPRÉCISION SOUS DIFFÉRENTES CONFIGURATIONS.

FIGURE 4.10 : L'IMAGE À GAUCHE EST OBTENUE SANS LE COMPORTEMENT DE MOUVEMENT ET L'IMAGE À DROITE EST OBTENUE EN UTILISANT LE COMPORTEMENT DE MOUVEMENT. .61

FIGURE 4.11 : PRÉCISION SOUS DIFFÉRENTES CONFIGURATIONS AVEC UN OBJET EN MOUVEMENT

FIGURE 4.12 : RÉPARTITION DE L'IMPRÉCISION SOUS DIFFÉRENTES CONFIGURATIONS AVEC UN OBJET EN MOUVEMENT.

FIGURE 4.13 : L'IMAGE À GAUCHE EST OBTENUE SANS LE COMPORTEMENT DE MOUVEMENT ET L'IMAGE À DROITE EST OBTENUE EN UTILISANT LE COMPORTEMENT DE MOUVEMENT. .......64

FIGURE 4.14 : FONCTIONNEMENT DU MODULE DE RECONNAISSANCE DE COMPORTEMENTS ERRONÉS

FIGURE 4.15 : FONCTIONNEMENT DU SYSTĖME DE LOCALISATION AVEC DU MATÉRIEL RFID ACTIF EN EXTÉRIEUR. LE TRAJET DE RÉFÉRENCE EST EN ROUGE ET LE TRAJET CALCULÉ AVEC L'ALGORITHME DE POSITIONNEMENT EST EN JAUNE. LES CARRÉS EN BLEU REPRÉSENTENT DES ANTENNES RFID.

FIGURE 4.16 : EMPLACEMENT DES 16 ANTENNES RFID (LES CARRÉS EN BLEU) LORS DES EXPÉRIMENTATIONS À LA ZONE PORTUAIRE.

FIGURE 4.17 : FONCTIONNEMENT DU SYSTĖME DE LOCALISATION AVEC DU MATÉRIEL RFID ACTIF EN EXTÉRIEUR. LE TRAJET GPS EST EN ROUGE ET LE TRAJET CALCULÉ AVEC L'ALGORITHME DE POSITIONNEMENT EST EN JAUNE. LES CARRÉS EN BLEU REPRÉSENTENT DES ANTENNES RFID.

FIGURE 4.18 : RÉPARTITION DE L'IMPRÉCISION SOUS DIFFÉRENTES CONFIGURATIONS LORS DES EXPÉRIMENTATIONS SUR LE TERRAIN DE FOOTBALL. 


\section{LISTE DES TABLEAUX}

TABLE 4.1 : TABLEAU RÉCAPITULATIF DES DIFFÉRENTS SYSTĖMES DE LOCALISATION 


\section{CHAPITRE 1}

\section{INTRODUCTION}

\subsection{Contexte}

Le Canada vit actuellement une crise démographique importante liée au vieillissement accéléré de sa population [1]. Plusieurs conséquences découlent de cet état de fait : problèmes budgétaires liés aux soins de santé, pénurie de personnel qualifié dans le domaine médical, manque de ressources pour les soins à domicile dédiés aux personnes en perte d'autonomie, etc. Par ailleurs, les personnes âgées, dont une proportion grandissante souffre de troubles cognitifs (ex. maladie d'Alzheimer), souhaitent majoritairement demeurer à domicile [2]. Les gouvernements abondent dans ce sens pour des raisons aussi bien sociales qu'économiques. En fait, ce maintien est clairement souhaitable, car il contribue à l'amélioration de la qualité de vie en permettant la désinstitutionnalisation. Il permet aux personnes de vivre une vie aussi normale que possible sans ségrégation dans les hôpitaux et une existence digne et un plein accès à la citoyenneté et à leur autonomie. Or, le maintien à domicile des personnes atteintes de troubles cognitifs comporte de nombreux risques qu'il est nécessaire de contrôler [3]. L'environnement physique et humain des résidences se doit donc d'être adapté, voire augmenté à l'aide de la technologie, afin de répondre aux besoins, de pallier aux incapacités cognitives et physiques, d'assurer la sécurité et de supporter les résidents dans leurs activités quotidiennes. C'est dans ce contexte d'urgence que depuis une dizaine d'années, un peu partout à travers le monde, une communauté [4-9] de scientifiques cherche activement des réponses technologiques à ce problème grâce au concept $d$ ' « habitat intelligent » [10].

L'habitat intelligent [5] s'inscrit dans le récent courant de pensée issu de l' « intelligence artificielle ambiante $»$, et réfère à une tendance voulant que l'on miniaturise un ensemble de 
dispositifs électroniques (capteurs et effecteurs) pour ensuite les intégrer dans plusieurs objets du quotidien (portes d'armoire, cuisinière, lampes, etc.), de manière transparente pour la personne, dans le but de fournir une assistance ponctuelle aux occupants et aux aidants en fonction des informations recueillies et de l'historique des données accumulées. Par exemple, une des problématiques fondamentales inhérentes au développement de ce type de technologies d'assistance adaptées concerne la reconnaissance et la prédiction des comportements [11] normaux ou anormaux des occupants (ex. personnes Alzheimer) effectuant leurs activités de la vie quotidienne (préparer un repas, laver les mains, allumer la télévision, etc.) à l'intérieur de l'habitat. Ces technologies visent ainsi, d'une part, une assistance directe au patient en-cas, par exemple, d'un oubli dû à une perte de mémoire (ex. oubli de la cuisinière allumée). Dans ce cas, le système intelligent peut effectuer une guidance/rappel à l'aide, par exemple, de la voix (message vocal), de la vidéo (message vidéo), du système d'éclairage (faire scintiller la lumière de la cuisinière) [12]. Cette assistance doit être adaptée au profil de l'individu (habitudes, habiletés, troubles cognitifs, etc.) de façon à maximiser l'efficacité de l'intervention. D'autre part, ces technologies visent aussi à assister l'aidant, lui permettant de diminuer la lourdeur de sa charge en monitorant, par exemple, la fuite et les comportements anormaux de la personne durant la nuit, lui permettant de passer une bonne nuit de sommeil.

Afin d'être en mesure d'accomplir ces tâches importantes de soutien technologique, le système de l'habitat intelligent doit d'abord être capable d'identifier les activités de la vie quotidienne (AVQ) effectué par le résident en inférant à partir des événements de base observés et à partir des événements captés par les senseurs dissimulés dans l'environnement. Cette problématique est appelée, dans le domaine de l'intelligence ambiante, la « reconnaissance d'activités » [13], sous-domaine bien connu et actif de la recherche en intelligence artificielle. Bien qu'un grand nombre de données de diverses natures (logiques, temporelles, spatiales, etc.) 
en provenance des capteurs puissent être exploitées par les approches de reconnaissance dans leur processus inférenciel, dans le contexte spécifique des habitats intelligents, les informations à caractères spatiales (position, mouvement, orientation, etc.) liées aux objets du quotidien jouent un rôle fondamental dans le cycle de reconnaissance [14]. L'obtention de ces informations, les plus précises possible, constitue donc un défi clé pour la mise en œuvre des systèmes de reconnaissance d'activités (et des erreurs cognitives) dans les habitats intelligents. Cette sous problématique se caractérise par la capacité de mettre en œuvre un système de localisation permettant le suivi des principaux objets en temps réel de manière fiable, efficace, ayant la plus grande précision possible [15]. Ce mémoire adresse principalement cette problématique.

\subsection{Problématique ciblée dans ce mémoire}

Au cours des dernières années, de nombreux chercheurs ont proposé des solutions pour la mise en œuvre de systèmes de positionnement basés sur différentes technologies comme les systèmes s'appuyant sur le GPS [16], les capteurs à ultrasons [17], les caméras vidéo [18] et enfin les systèmes basés sur l'identification par radio fréquence (RFID). Ces différentes approches et technologies proposent différentes façons de répondre au problème de la localisation et de suivi des principaux objets mobiles de l'habitat en temps réel. Toutefois, dans le contexte des habitats intelligents, la technologie RFID semble s'être imposée comme l'une des meilleures solutions actuellement disponibles en raison de sa robustesse, de son prix faible et de sa souplesse [15]. En général, un système RFID se compose de trois éléments (voir Figure 1.1) : les étiquettes d'identification par radio fréquence, d'au moins une antenne et d'un module de collecte de données. Un système RFID passif fonctionne comme suit. Tout d'abord, l'antenne RF (Radio Fréquence) émet un rayonnement d'ondes électromagnétiques. Ensuite, si une étiquette RFID est située dans la zone de couverture de l'antenne, elle intercepte le signal, 
s'active et la puce interne en renvoie un nouveau à l'antenne. Finalement, l'antenne d'émission capte ce nouveau signal et renvoie les informations contenues dans le signal au module de collecte. Ces données peuvent être utilisées par un algorithme pour déduire des informations spatiales (ex. leur position) sur les objets étiquetés.

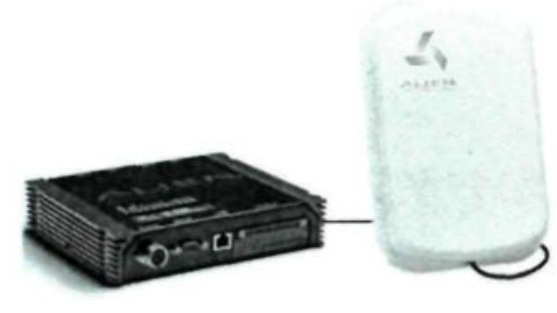

Lecteur RFID

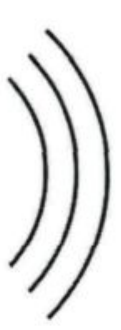

Antenne RFID

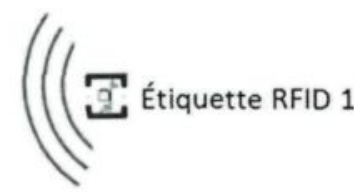

$((($ É Étiquette RFID 2

Figure 1.1: Système RFID - antennes, module de collecte et étiquettes (adapté de [19]).

Les étiquettes d'identification par radiofréquence sont subdivisées en trois familles : les étiquettes actives, semi-actives et passives. Les étiquettes actives et semi-actives sont alimentées par une batterie et ont souvent une mémoire interne réinscriptible. Par conséquent, peu importe la force des signaux transmis par les antennes, les puces RFID seront toujours en mesure d'envoyer un signal à une puissance constante. Par conséquent, les puces actives ont une plus grande portée ainsi qu'une stabilité élevée dans la force du signal. En outre, les systèmes de positionnement avec ce type de puce sont beaucoup plus avancés. Par exemple, HeikimianWilliams et al. [20] ont obtenu des résultats très précis avec une approche simple basée sur le déphasage entre les signaux reçus à partir de deux antennes distinctes. Pour estimer la différence de phase, ils ont utilisé un logiciel couplé avec des horloges précises. Néanmoins, dans le cas de l'assistance à l'intérieur d'un habitat intelligent, il y a des besoins particuliers que la technologie active ne peut pas répondre. Tout d'abord, il est nécessaire que le système soit autonome et, donc, qu'il nécessite peu d'entretien. À ce sujet, les étiquettes passives ont une durée de vie théoriquement illimitée et n'ont pas besoin d'alimentation externe. Deuxièmement, nous devons 
réduire l'intrusion et les étiquettes actives sont d'une taille beaucoup plus imposante que leurs homologues passives. En outre, il est possible d'incorporer des étiquettes RFID passives dans des objets de la vie quotidienne tels que des tasses, des assiettes, etc. Enfin, si nous voulons que cette technologie soit adoptée, il faut penser au coût matériel. À ce propos, alors que les étiquettes passives coûtent seulement quelques cents, les étiquettes actives et semi-actives sont dans une tranche de prix beaucoup plus élevée. Pour toutes ces raisons, le développement d'une solution de localisation RFID précise avec des étiquettes passives pour l'habitat intelligent constitue une avenue privilégiée et très intéressante pour permettre la mise en œuvre d'une reconnaissance d'activités visant une assistance cognitive.

\subsection{Travaux antérieurs liés au positionnement par RFID}

Les chercheurs qui travaillent sur les systèmes de localisation RFID s'appliquent souvent dans des secteurs industriels et commerciaux autres que les habitats intelligents. À titre d'exemple, on peut citer l'exploitation de ces approches pour la traçabilité des denrées alimentaires [21], pour les inventaires automatisés [22] et plus récemment, pour le contrôle d'accès dans les grands événements [23]. C'est pourquoi la majorité de la littérature scientifique met l'accent sur les systèmes exploitant les balises actives. Néanmoins, plusieurs chercheurs ont étudié les systèmes RFID passifs. Par exemple, Hähnel et al. [24], Vorst et al. [25], Joho et al. [26] et Chawla et al. [27] ont tous développé différentes approches qui produisent des résultats relativement intéressants. Cependant, chacun de ces travaux s'appuie sur l'utilisation de balises de référence pour localiser les objets. Une balise de référence est une étiquette qui est placée à un endroit précis et connu pour fournir un point de repère pour localiser les autres. Par ailleurs, dans certain cas, ils ont utilisé plus de 300 balises de références pour leurs expérimentations. Cela rend leurs méthodes difficiles à mettre en œuvre dans contexte d'un habitat intelligent, où l'on 
souhaite minimiser le nombre d'étiquettes présentes dans l'environnement et limiter leur utilisation aux objets mobiles. Par ailleurs, Zhang et al. [28] présentent une nouvelle approche utilisant les angles d'arrivés avec des étiquettes passives, ce qui est un concept très intéressant, mais les résultats obtenus sont peu précis. En simulation, ils ont obtenu des résultats au mètre près, ce qui est insuffisant pour effectuer la localisation de l'objet sur un comptoir de cuisine, par exemple. Enfin, seulement quelques groupes de chercheurs $[28,29]$ ont récemment tenté de développer de nouvelles méthodes de localisation pour les étiquettes RFID passives sans balises de références et chacun de ces travaux présente des limitations importantes à l'égard de notre contexte applicatif, principalement au niveau de la précision et de la robustesse. Le chapitre 2 traitera de celles-ci.

\subsection{Contribution du mémoire}

Dans l'objectif d'apporter une contribution à la recherche scientifique liée aux technologies pour le soutien des personnes en perte d'autonomie, ce mémoire propose de formaliser et d'évaluer un nouveau modèle multicouche et un nouvel algorithme de localisation précis des étiquettes RFID passives capable d'être appliqué dans un contexte de reconnaissance d'activités et de détection des erreurs pouvant être commises par un résident au sein d'un habitat intelligent. La contribution de ce mémoire revêt un volet théorique, un volet pratique, ainsi qu'un volet expérimental. Tout d'abord, au niveau théorique et fondamental, ce travail propose un nouveau modèle amélioré de positionnement basé sur une approche de multilatération à partir de plusieurs ellipses. Pour ce faire, un ensemble de nouveaux filtres a été appliqué pour réduire considérablement l'imprécision des forces de signaux. Par ailleurs, une autre originalité de l'approche consiste en l'introduction d'un nouveau filtre de post-traitement effectué sur la position résultante afin d'ajouter des règles qui définissent le comportement des mouvements des 
objets pour les rendre, entre autres, plus naturels. Côté pratique, notre contribution consiste en la mise en œuvre (l'implémentation) complète de ce nouveau système et en son déploiement dans un environnement réel d'habitat intelligent équipé d'antennes RFID et des objets de la vie quotidienne munis d'étiquettes RFID. Cette mise en œuvre permet de bien montrer l'applicabilité concrète des propositions théoriques formulées. Du côté expérimental, notre contribution consiste en une série d'expérimentations rigoureuses effectuées sur le nouveau système de localisation, permettant de démontrer son efficacité dans le contexte particulier de l'habitat intelligent. Pour ce faire, un protocole expérimental détaillé est présenté et les résultats obtenus en ce qui a trait à la précision du système de localisation sont analysés et comparés avec ceux des travaux antérieurs. Enfin, un déploiement de l'algorithme à l'intérieur d'un système complet de reconnaissance d'activités et d'erreurs cognitives a été réalisé en fin de projet. Les expérimentations préliminaires, montrant des résultats prometteurs, seront brièvement présentées.

\subsection{Méthodologie de la recherche}

Le projet de recherche présenté dans ce mémoire a été réalisé en suivant une méthodologie de recherche divisée en quatre étapes clés.

La première phase du projet visait à acquérir des connaissances sur le domaine de recherche ciblé en procédant à une revue de la littérature orientée sur le problème de la localisation par puces RFID en général $[16,30]$, et plus particulièrement ceux utilisant des étiquettes passives $[28,31,32]$. Ensuite, les systèmes de référence de la littérature en ce qui a trait à la localisation, tel que le bien connu LANDMARC [33] et le modèle de positionnement de Chen et al. [29] ont été étudiés en profondeur. La première partie de cette étape a permis d'acquérir une compréhension des problèmes inhérents à l'utilisation de puce RFID passive avec 
un système de localisation en général et d'entrevoir les contraintes spécifiques liées au contexte de l'habitat intelligent. La seconde partie de cette étape a permis une étude approfondie des différentes méthodes de localisation existante afin de mettre en évidence leurs forces et leurs faiblesses. La réalisation de cette étape clé a permis de formuler des hypothèses de solutions pertinentes qui ont conduit aux propositions formulées à la seconde étape.

La deuxième étape a consisté en l'élaboration d'une approche algorithmique par la formalisation d'un nouveau modèle de localisation par puces RFID passives pour résoudre les problèmes et répondre aux contraintes ciblées à la première étape. À cet effet, la méthode proposée se veut une extension ainsi qu'une amélioration du positionnement par trilatération [15, 29, 34, 35]. La raison principale justifiant ce choix est que les méthodes par trilatération sont flexibles, faciles à déployer et répondent aux contraintes imposées dans le contexte d'un habitat intelligent. Cette étape à mener à la contribution théorique du mémoire.

La troisième étape a consisté en une implémentation logicielle de ce nouveau modèle de localisation de puces RFID passive dans le contexte d'un habitat intelligent afin de valider ses performances et d'établir une base de comparaison avec les autres approches de positionnement et, en particulier, celles qui répondent aux mêmes contraintes liées à notre contexte applicatif. Pour ce faire, nous avons choisi de développer le système de positionnement en utilisant le langage de programmation Java, s'exécutant sur un ordinateur standard. Le programme est directement en communication avec une véritable infrastructure d'habitat intelligent et, donc, en communication en temps réel avec les capteurs et les effecteurs du laboratoire LIARA. De plus amples détails seront fournis dans le chapitre 4.

La dernière étape du projet de recherche a consisté en la validation du nouveau modèle créé et mis en œuvre. Cette étape avait pour but de vérifier l'applicabilité du modèle développé 
pour la localisation et de montrer son intérêt dans une orientation d'utilisation pour la reconnaissance d'activités et de comportements erronés. La première partie de cette phase était de mener une série d'expérimentations permettant d'évaluer le niveau de précision du système de positionnement avec des objets statiques et des objets en mouvement. Les résultats prometteurs obtenus seront décrits en détail au chapitre 4 et une analyse comparative de ceux-ci par rapport aux approches connexes de localisation existantes sera présentée. La dernière partie de cette étape consistait à effectuer une expérimentation préliminaire concernant la capacité du modèle à être utilisé dans un contexte de reconnaissance d'activités. Pour ce faire, l'algorithme a été intégré à un système de reconnaissance d'activités simple basé sur les relations topologiques entre les objets. Des scénarios d'activités basiques ont été testés et les résultats sont également présentés.

Enfin, il convient de mentionner que les travaux réalisés dans ce mémoire font l'objet d'un article scientifique qui a été accepté à la réputée conférence ACM-PETRA 2014.

\subsection{Organisation du mémoire}

Ce mémoire est organisé en cinq chapitres qui suivent la chronologie des étapes décrites dans la méthodologie de recherche.

Le premier chapitre, qui s'achève, se veut une introduction au contexte des travaux, à la problématique spécifique traitée dans le mémoire, ainsi qu'à la contribution proposée dans celuici. Il fournit une vue d'ensemble des travaux de recherche et permet au lecteur de bien voir le positionnement de la contribution proposée dans notre contexte applicatif.

Le deuxième chapitre présente une revue de la littérature sur les problèmes de la localisation, et plus particulière ceux liés à la technologie RFID. Ce chapitre se veut un état de 
l'art centré sur les principales approches de localisation identifiées dans la première phase de la recherche. Il débute par la description de la technologie utilisée et présente les bases théoriques des algorithmes de positionnement. Ensuite, le chapitre discute des impacts de la fluctuation imprévisible des forces de signaux sur les systèmes de positionnement qui l'utilisent dans le calcul de distance entre une puce et une antenne. D'ailleurs, plusieurs approches hybrides sont présentées pour éviter ce problème. Ensuite, les principales méthodes de localisation basées sur la technologie RFID sont décrites et analysées. Au terme du chapitre, nous concluons par une comparaison entre les avantages et les inconvénients des différents modèles tout en justifiant notre décision d'effectuer une amélioration ou extension des méthodes de positionnement par trilatération.

Le troisième chapitre consiste en une description détaillée de notre contribution théorique, qui prend la forme d'un nouveau modèle de localisation de puces RFID passive. Dans un premier temps, le fonctionnement des différents filtres du filtrage des forces de signaux sera présenté en commençant par le filtre de réduction des faux positifs et des faux négatifs, en passant par le filtre de réduction de crête et en finissant par le filtre de pondération par moyenne gaussienne. Ensuite, la nouvelle méthode de positionnement qui consiste en une multilatération par intersection géométrique de disques elliptiques sera décrite en détail. De plus, le filtrage effectué sur la position obtenue par multilatération, qui consiste en un ensemble de règles d'inférence régissant le comportement des mouvements des objets sera décrit. Le chapitre se termine par une revue étape par étape du fonctionnement du nouveau modèle de positionnement afin de bien comprendre le processus amenant à un positionnement précis et fiable à partir d'informations bruitées. 
Le quatrième chapitre examine la contribution du mémoire d'un point de vue pratique et expérimental. La première section présente la mise en œuvre de ce nouveau modèle de positionnement au sein d'un habitat intelligent basé sur la technologie RFID. La deuxième partie de ce chapitre traite du protocole utilisé pour valider le modèle proposé et présente une analyse comparative des résultats obtenus avec les principales approches dans le domaine. La dernière section décrit l'intégration à un algorithme de reconnaissance d'activités visant l'assistance cognitive et traite des résultats préliminaires obtenus.

Finalement, le cinquième et dernier chapitre permet de conclure le mémoire en présentant un compte rendu détaillé du projet de recherche en mettant en évidence la contribution de ce travail en rapport aux recherches précédentes. Ce chapitre abordera également les limites de l'approche et offrira des pistes pour les travaux futurs découlant de cette recherche. Le chapitre se terminera par une évaluation plus personnelle de cette expérience initiatique dans le monde de la recherche scientifique. 


\section{CHAPITRE 2}

\section{APPROCHES DE LOCALISATION EN ENVIRONNEMENT INTÉRIEUR}

\subsection{Introduction}

Au fil des années, diverses technologies ont été envisagées pour la localisation des objets à l'intérieur d'un habitat. Plusieurs chercheurs ont envisagé d'exploiter le système de positionnement global (GPS) [36]. Ses performances pour les applications extérieures n'ont plus besoin d'être démontrées. Cependant, la technologie GPS est confrontée au problème prohibitif de la pénétration de la paroi ce qui restreint son utilisation pour les applications au sein d'un habitat intelligent. Il existe également des modèles de localisation basés sur des capteurs d'ondes ultrasonores. Bien qu'il y ait des systèmes notables qui fonctionnent bien en exploitant cette technologie, tel que le système de localisation BAT [14], ils souffrent de difficultés importantes dans notre contexte. Premièrement, ce système est compliqué à mettre en œuvre à la fois sur le plan matériel et logiciel. Deuxièmement, le suivi de plusieurs objets est coûteux, car ceci nécessite de fixer un émetteur sur chaque objet qui doit être localisé à l'intérieur de l'habitat intelligent. Enfin, la complexité de calcul de ces systèmes est élevée ce qui rend son utilisation difficile, voire impossible, en situation de temps réel.

Une autre catégorie de méthode est celle basée sur la caméra vidéo pour effectuer cette tâche [37]. C'est de loin la technique fournissant les informations les plus complètes sur l'objet suivi. Toutefois, ces systèmes sont souvent moins souples, car les conditions environnementales doivent être soigneusement calibrées. Par conséquent, leur portabilité est limitée. En outre, l'utilisation de caméras vidéo pose des problèmes de confidentialité et n'est pas bien acceptée 
par les différents groupes de personnes impliquées dans le processus d'assistance (professionnels de la santé, des médecins, des résidents, des membres de la famille, etc.). En fait, dans notre contexte d'habitat intelligent, il a été démontré que l'intrusion pourrait aggraver la détresse des sujets atteints d'Alzheimer.

\subsection{Identification par radio fréquence (RFID - Radio-Frequency Identification)}

Typiquement, un système RFID se compose de trois éléments: les étiquettes d'identification par radiofréquences, au moins une antenne RF et le module de collecte de données. Les étiquettes RF sont subdivisées en trois familles : étiquettes actives, semi-actives et passives. Ces familles possèdent des caractéristiques de base très distinctives offrant différentes alternatives pour répondre à un large éventail de problèmes. Alors que les étiquettes actives sont surdimensionnées, leurs homologues passifs sont discrets et peuvent même être intégrés dans un objet. Par conséquent, ils sont plus robustes et peuvent fonctionner de façon autonome pendant une longue période de temps. Cependant, puisque les étiquettes actives sont alimentées par batterie, elles peuvent atteindre une portée et une précision considérablement plus élevées que les passives. Par exemple, le travail de Cory Hekimian-Williams [20] atteint presque une précision de l'ordre du millimètre avec la technique de différence de phase, qui est loin d'être possible avec la technologie passive. D'un autre côté, les batteries des étiquettes actives nécessitent une maintenance rapide et un certain coût ce qui restreint leurs usages. Le problème de la localisation avec des étiquettes autoalimentées est beaucoup plus simple et c'est pourquoi nous pouvons trouver un grand nombre de systèmes et de travaux exploitant cette méthode [24, 38, 39]. Toutefois, dans le cas de l'aide intelligente à domicile, nous avons besoin d'une technologie non intrusive qui nécessite peu d'entretien et qui est raisonnablement peu coûteuse. Les étiquettes passives peuvent coûter aussi peu que quelques centimes, et elles peuvent même 
être directement intégrées dans les objets de la vie quotidienne (tasse, assiette, etc.). La technologie passive est, donc, plus appropriée pour l'aide intelligente à domicile.

Pour effectuer la localisation d'objets grâce à la technologie RFID, plusieurs techniques peuvent être envisagées [36]. Nous pouvons regrouper les différents algorithmes en trois grandes catégories : ceux basés sur le délai entre l'émission et la réception (time-of-arrival), ceux basés sur la différence de phase et ceux basés sur l'indication de la puissance du signal reçu (RSSI). Les deux premiers, ceux basés sur le délai entre l'émission et la réception et sur la différence de phase, sont largement utilisés avec des balises actives et sont très difficiles à exploiter avec des étiquettes passives. D'ailleurs, il est facile en général de transformer le RSSI en une information de distance à partir d'une antenne à l'aide de l'équation de transmission de Friis [16] pour calculer la distance d'une étiquette par rapport à une antenne. La version la plus simple de cette équation est :

$$
P_{r}=P_{t} * \frac{G_{t} * G_{r} * A^{2}}{16 * L * \pi^{2} * d^{2}}
$$

où $P_{r}$ est la puissance reçue par l'antenne réceptrice, $P_{t}$ est la puissance d'entrée de l'antenne émettrice, $G_{t}$ est le gain de l'antenne émetteur, $G_{r}$ est le gain de l'antenne récepteur, $L$ est le facteur de perte du système, $A$ la longueur d'onde et $d$ est la distance entre l'antenne émettrice et l'antenne réceptrice. Le 'gain' d'une antenne est sa capacité à concentrer le signal qu'elle reçoit pour en augmenter la puissance dans une certaine direction. Avec cette relation, si la force du signal reçue par l'antenne est connue, on peut calculer la distance qui la sépare de l'étiquette. Naturellement, il y a d'autres méthodes pour calculer la distance approximative avec les RSSI mais le principe est similaire. À partir de plusieurs informations de distance, la manière classique pour trouver la position est d'effectuer un calcul de trilatération. En deux dimensions, si un objet 
est détecté par trois antennes, nous pouvons utiliser la technique avec des cercles telle qu'illustrée à la figure 2.1 ci-dessous. Soient $r_{1}, r_{2}$ et $r_{3}$ les distances estimées pour les trois antennes. Sachant que la première antenne est située en position $\left(x_{1}, y_{1}\right)$, la seconde en $\left(x_{2}, y_{2}\right)$ et la dernière en $\left(x_{3}, y_{3}\right)$, on construit alors 3 équations (une par antenne) qui représentent des cercles centrés en $\left(x_{1}, y_{1}\right)$ de rayon $r_{1}$, en $\left(x_{2}, y_{2}\right)$ de rayon $r_{2}$ et en $\left(x_{3}, y_{3}\right)$ de rayon $r_{3}$. On a alors les systèmes d'équations suivants :

$$
\begin{aligned}
& \left(x-x_{1}\right)^{2}+\left(y-y_{1}\right)^{2}=r_{1}^{2} \\
& \left(x-x_{2}\right)^{2}+\left(y-y_{2}\right)^{2}=r_{2}^{2} \\
& \left(x-x_{3}\right)^{2}+\left(y-y_{3}\right)^{2}=r_{3}^{2}
\end{aligned}
$$

Ensuite, il ne reste qu'à calculer le point ou les points d'intersection entre des cercles.

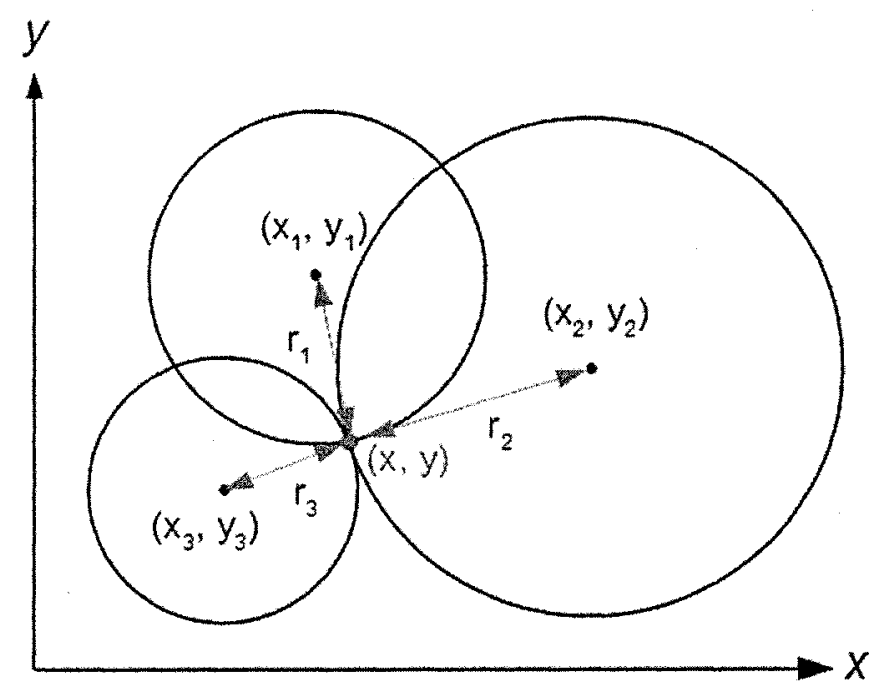

Figure 2.1 : Intersection de 3 cercles (adapté de [40])

Puisqu'une simple trilatération basée sur les RSSI est très imprécise avec la technologie passive, de nombreux systèmes actuels utilisent la stratégie intuitive de localisation par proximité [18]. L'idée derrière cette technique consiste à déployer un grand nombre d'antennes et de calibrer leur portée afin de réduire autant que possible le chevauchement. Un objet qui est dans la zone de détection d'une antenne est censé être dans la même position que cette antenne. Lorsque 
plusieurs antennes détectent un objet, la position estimée est celle de l'antenne qui reçoit le signal le plus puissant. Même si elle est simple, cette technique fonctionne très bien et est actuellement la méthode la plus robuste. L'inconvénient est le manque de précision de l'information sur la position des étiquettes. Ceci pourrait être un obstacle pour le développement des services plus avancés correspondant au contexte et à l'environnement d'un habitat intelligent. L'algorithme des k-plus proches voisins [16] est une autre méthode de localisation par proximité. Comme on peut le voir à la figure 2.2 , l'idée derrière cette méthode est de délimiter une zone dans laquelle le nœud cible se situe en déterminant quels sont ses plus proches voisins (les nœuds de référence ayant reçu les signaux les plus élevés). Ensuite, la position est estimée en effectuant une moyenne pondérée des positions des $K$ plus proches voisins à partir des différences entre les forces de signaux obtenues à ces $K$ points. Cette technique nécessite soit un grand nombre d'antennes soit une multitude de balises de référence pour permettre un bon niveau de précision. Donc, cette autre méthode de localisation par proximité est peu envisageable dans le contexte d'un habitat intelligent. En conclusion, ces méthodes ne sont pas suffisantes pour avoir une localisation précise et fiable dans le contexte particulier d'un habitat intelligent. C'est pourquoi nous devons s'informer sur de meilleures méthodes.

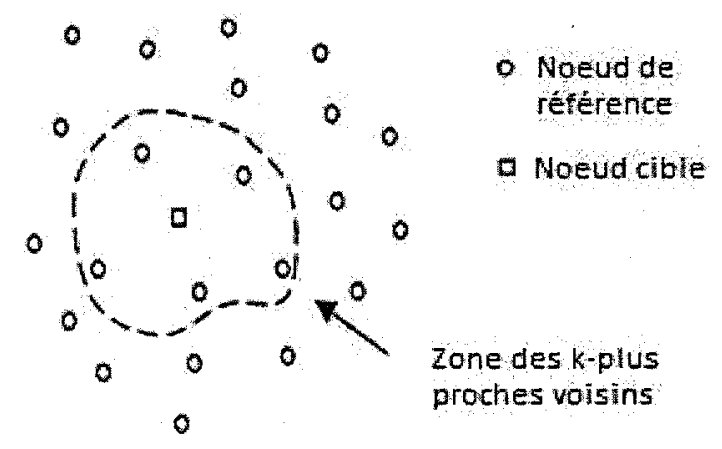

Figure 2.2: K-Plus proches voisins (adapté de [16]) 


\section{3 Évaluation des impacts des RSS sur un système de localisation}

Les méthodes de positionnement à partir des forces de signaux utilisent la relation de l'atténuation de la force du signal en fonction de la distance pour obtenir un modèle mathématique de propagation. Cependant, les forces de signal radio ont la réputation de fluctuer imprévisiblement. Telle qu'illustrée à la figure 2.3 ci-dessous, cette imprévisibilité produit une corrélation imparfaite entre les forces de signaux et les distances [41]. Une corrélation imparfaite rend imprécis le calcul d'une distance à partir d'une force de signal et une corrélation trop faible rend difficile l'obtention d'un modèle mathématique de propagation. Par ailleurs, aux figures 2.3 et 2.4 on remarque que le niveau d'erreur augmente en fonction de la distance et qu'il existe un intervalle de distance où le niveau de précision est optimal tout dépendamment du matériel et du contexte d'utilisation.

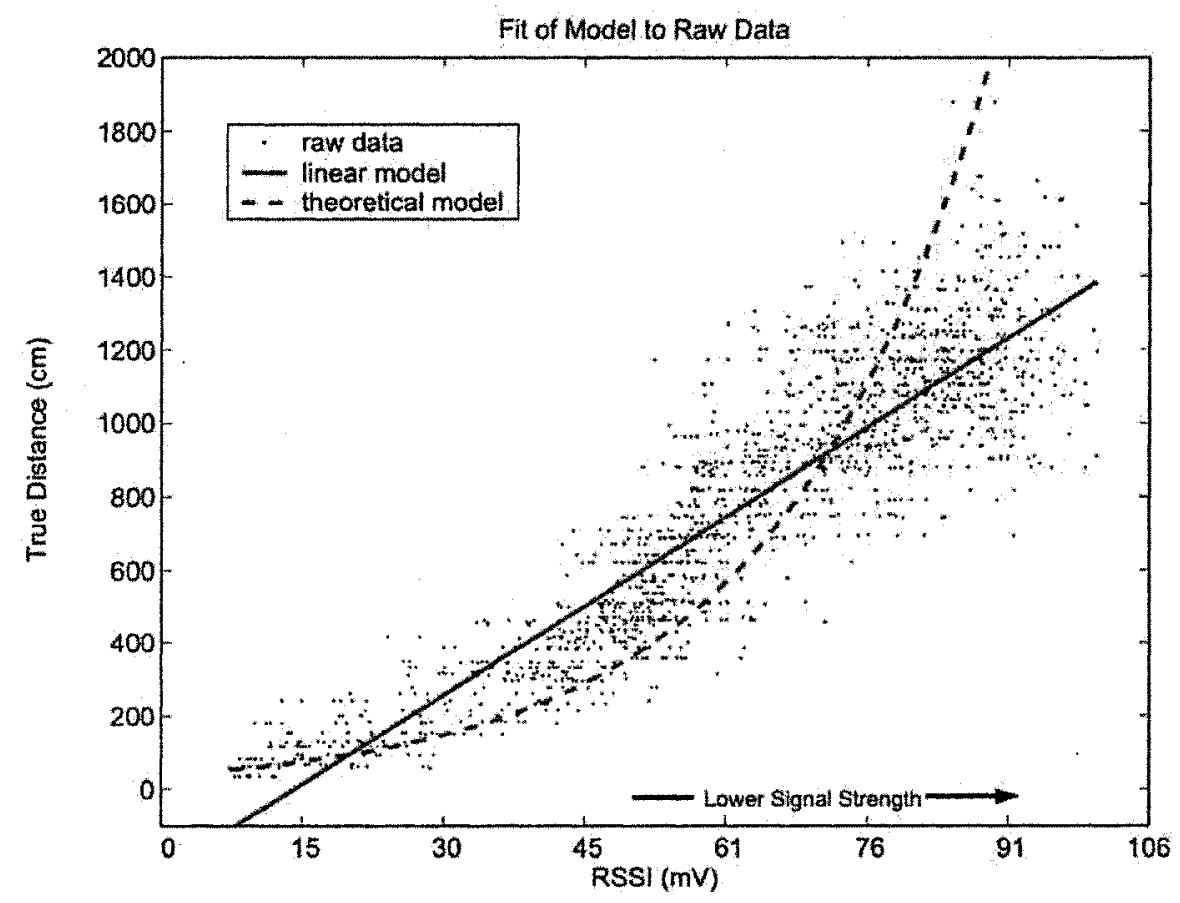

Figure 2.3 : Corrélation des RSSI en fonction des distances [41] 


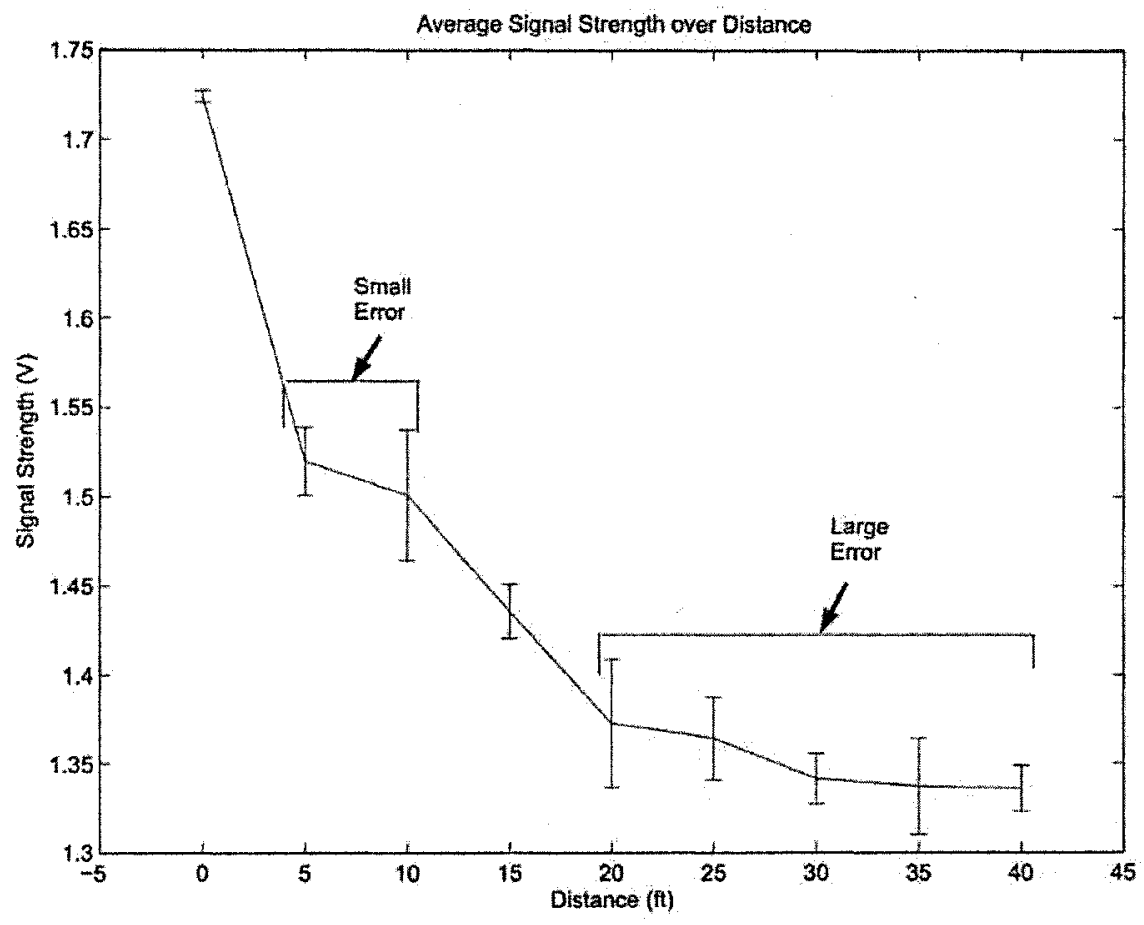

Figure 2.4: Imprécision des RSSI en fonction de la distance [41]

De plus, les RSS (force de signal radio) sont sensibles à l'environnement. Donc, une solution de localisation précise et fiable à partir des RSSI (indication sur la force du signal radio) doit prendre en considération les caractéristiques d'atténuation et de propagation des forces de signaux. Une de ces caractéristiques est l'habileté du signal à passer à travers certains matériaux [30]. Cela inclut les humains et les animaux, les liquides, plusieurs sortes de métaux et les autres matériaux. En fait, chaque type de matériel a son propre taux d'absorption et de réflexion des ondes radio. La réflexion des ondes radio cause un envoi de plusieurs signaux distincts avec chacun leur propre puissance. Pour mieux comprendre ces phénomènes, il convient de préciser qu'une onde lumineuse est une onde électromagnétique dont la longueur d'onde correspond au spectre visible. Les ondes radio sont également des ondes électromagnétiques, mais leur longueur d'onde correspond à un spectre différent. Par conséquent, tous les phénomènes observés avec les ondes lumineuses ont des équivalences avec les ondes radio. De plus, les 
étiquettes RFID peuvent avoir plusieurs fréquences de fonctionnement. Chaque fréquence a aussi sa propre réaction face à l'environnement. Par exemple, les ultras hautes fréquences (UHF) ont l'avantage d'avoir une plus grande portée mais passe plus difficilement au travers des liquides et donc, des humains. De plus, ce n'est pas seulement les ondes émises par les étiquettes qui sont altérées, les ondes envoyées par les antennes le sont aussi. C'est entre autres pour cette raison que les étiquettes RFID actives causent moins de problèmes. Bref, dans le monde réel, ce qui devrait fonctionner correctement de manière théorique se comporte rarement aussi bien dans la pratique. Donc, bien qu'il existe des techniques mathématiques de localisation (triangulation et trilatération circulaire) qui fonctionnent avec une précision infinie de manière théorique, en pratique cela s'avère souvent bien plus complexe. Pour conclure, dans le but de faire une localisation précise et fiable, on doit prendre en considération les phénomènes suivants : la multipropagation des ondes radio, l'absorption ou l'atténuation des ondes, les réactions différentes en fonction de la fréquence de l'onde utilisée et il faut aussi prendre en considération que les caractéristiques de l'environnement peuvent changer.

\subsection{Les approches hybrides ou sans la technologie RFID}

\subsubsection{L'utilisation des ultrasons}

Addlesee \& al. [17] est probablement l'un des systèmes de localisation non-RF le plus renommé. Leur système repose sur la seule exploitation des capteurs à ultrasons. Il atteint une précision considérable $(\approx 3 \mathrm{~cm})$ dans des conditions idéales, mais nécessite un déploiement dense de récepteurs coûteux installés dans le plafond de la zone ciblée. Choi et al. [42] combine la technologie des ultrasons avec des étiquettes RFID passives de référence pour localiser un robot. Une étiquette de référence est une balise qui est statique et fixe à un endroit prédéfini. Il est utilisé pour aider l'algorithme de localisation à déduire la position d'une étiquette mobile en 
comparant sa position estimée avec les balises de référence. Ils donnent une précision beaucoup plus élevée que toute autre approche RFID $(\approx 1-3 \mathrm{~cm})$. Cependant, les systèmes à base d'ultrasons sont limités par des conditions environnementales telles que le bruit, les obstructions matérielles entre la source et le capteur, etc.

\subsubsection{La combinaison de la vision ou d'un laser avec la technologie RFID}

Hähnel \& al. [24] est un autre travail de positionnement majeur que met l'accent sur la localisation de robot. Ils unissent un lecteur RFID avec un numériseur à laser qui est installé à bord d'un robot mobile. Une phase d'apprentissage de la carte est tout d'abord réalisée avec le laser. Ensuite, il est utilisé en combinaison avec des étiquettes en positions fixes et connues pour localiser un robot. En dépit de sa nouveauté, leur système obtient une erreur moyenne élevée du positionnement de l'ordre de 1-10 mètres. En outre, il doit également gérer la contrainte de la ligne de visée du laser qui peut être obstruée par un obstacle. Heesung \& Kyuseo [43] et plus tard Milella et al. [44] ont combiné des capteurs de vision (caméra) avec la technologie RFID pour atteindre une précision de l'ordre de $20 \mathrm{~cm}$. Sample $\&$ al. [31] est un autre travail qui exploite les capteurs de vision. Ils améliorent les étiquettes avec des LEDs (diode électroluminescente) pour permettre à un robot équipé d'une caméra de localiser plus précisément la balise dans l'environnement.

\subsubsection{La combinaison de la technologie RFID avec un capteur de mouvement}

Récemment, Parr \& Miesen [45] ont introduit une nouvelle méthode de suivi d'étiquettes RFID en fusionnant une unité de mesure inertielle à un lecteur portatif. Leur technique utilise des données d'accélération sans connaissance de la position de l'antenne pour atteindre un positionnement précis à un coût raisonnablement bas. Pourtant, le matériel nécessaire pour 
supporter ce système (un accéléromètre ainsi qu'une batterie) est trop encombrant pour être intégré aux objets à l'intérieur de l'habitat.

\subsubsection{Bilan sur les approches hybrides}

Bien que les approches sans la technologie RFID et les approches hybrides donnent un meilleur rendement que l'utilisation seule de la technologie RFID, elles sont sans doute moins adaptées à de nombreuses situations. D'abord, elles sont plus coûteuses au point que certaines d'entre elles restent principalement inutilisées après de nombreuses années. Deuxièmement, elles s'appuient maladroitement sur une technologie qui souffre d'une haute intrusion (les caméras

sont particulièrement prohibitives dans notre cas). Troisièmement, ces approches imposent souvent une contrainte sur une ligne de vue directe que les technologies par radiofréquence permettent souvent d'éviter. Quatrièmement, aucun de ces systèmes offrent une robustesse plus élevée que les étiquettes RFID passives car celles-ci peuvent être mises dans un lave-vaisselle et ne nécessitent aucunement l'utilisation d'encombrantes batteries. Ces systèmes sont aussi plus lents et plus difficiles à installer que les méthodes RFID simples. En somme, ils ne présentent pas les avantages qui rendent la technologie RFID si attrayante pour la localisation dans le contexte de l'assistance à l'intérieur d'un habitat intelligent.

\subsection{La localisation basée sur la technologie RFID}

De nombreuses techniques RFID sont basées sur le système de localisation bien connu LANDMARC [33]. Ce système introduit le concept de localisation à l'aide de balises de références placées à des emplacements stratégiques. Le travail de Jin et al. [39] est l'un d'entre eux. Ils exploitent la technologie RFID active et ont développé une version améliorée du système LANDMARC en ne sélectionnant que quelques balises à proximité de la cible. Ils atteignent un positionnement précis à $72 \mathrm{~cm}$ près. De la même manière, Vorst et al. [25] utilisent des étiquettes 
RFID passives et un lecteur intégré pour localiser des objets mobiles. Ils exploitent une technique qui consiste en un filtre de particules (FP) pour estimer l'emplacement à partir d'un modèle probabiliste précédemment appris. Ils peuvent atteindre une précision de $20-26 \mathrm{~cm}$. Bekkali \& al. [46] proposent également une approche probabiliste mais en utilisant le filtre de Kalman. Ils atteignent une erreur de localisation de l'ordre de 50 à $100 \mathrm{~cm}$. L'inconvénient majeur de ces deux systèmes est la durée trop élevée du temps de calcul. Lei et al. [32] ont abordé cette question en combinant le FP avec la localisation du barycentre à partir d'un ensemble pondéré de points. Ils passent d'une méthode à l'autre en fonction de l'estimation de la vitesse de l'objet suivi. Dans des conditions idéales, ils localisent une antenne avec une erreur moyenne de $20 \mathrm{~cm}$ tout en augmentant fortement la vitesse du processus. Joho et al. [26] présentent un modèle qui utilise la force de signal (RSSI), l'orientation de l'antenne et des étiquettes de références. Un lecteur mobile rassemble des informations pour les comparer avec la position réelle. L'erreur de localisation est d'environ $35 \mathrm{~cm}$. Chawla \& Robins [27] ont développé un modèle basé sur la variation de la puissance de l'antenne pour estimer la distance des balises de référence à proximité. Ils ajustent progressivement la force (le nombre de décibels) de l'antenne jusqu'à ce que la balise soit à portée. Par la suite, ils utilisent la distance entre l'antenne et de nombreuses balises pour localiser un robot mobile. Leur approche donne une précision variant de 18 à $35 \mathrm{~cm}$. Pour conclure, bien que certaines de ces approches offrent de très bons résultats, ils exploitent toutes des techniques nécessitant un grand déploiement de balises de référence. Il fonctionne bien pour la localisation d'un robot. Cependant, il n'est pas toujours possible de placer un grand nombre de balises dans un environnement. Ils seraient également trop intrusifs pour l'assistance de l'habitat intelligent qui nécessite de limiter les changements sur l'environnement de la personne. De plus, une installation soignée de balises de référence à des positions stratégiques est une tâche complexe et fastidieuse. En outre, dans notre 
cas, nous voulons parvenir à localiser des objets de la vie quotidienne (tasse, livre, etc.). Les techniques décrites précédemment ont été conçues dans le but de localiser une antenne à partir d'un grand nombre d'étiquettes. Cependant, les antennes sont beaucoup trop grosses pour être intégrées dans des objets. Par conséquent, ces modèles ne conviennent pas à nos besoins.

Brchan \& al. [47] utilisent la technologie active pour se concentrer sur le problème de la localisation en temps réel qui a été largement ignoré dans la littérature. Ils utilisent des modèles de propagations multiples pour améliorer la précision de force de signaux (RSSI) en utilisant des étiquettes de référence. Hekimian-Williams \& al. [20] est peut-être le système de localisation le plus précis parmi tous ceux qui sont basés sur la technologie RFID active. Ils exploitent les différences de phase pour atteindre une précision au millimètre près dans des conditions parfaites. Cependant, ils ignorent des facteurs clés tels que la sensibilité d'une étiquette. De plus, la différence de phase est difficilement utilisable avec la technologie passive. Bien que les étiquettes actives puissent être une bonne option pour obtenir un signal sur une plus longue distance, ils souffrent de nombreuses faiblesses. Tout d'abord, ils sont de dimension plus élevée que leurs homologues passives. Deuxièmement, elles sont beaucoup plus coûteuses. Enfin, elles dépendent d'une batterie qui nécessite un entretien, en temps opportun, qui devrait être évité dans le contexte de l'habitat intelligent. D'autres équipes de recherche ont tenté d'aller plus loin par la mise en œuvre d'un système de localisation en 3D $[48,49]$. Bien qu'ils aient obtenu une précision intéressante $(10$ à $90 \mathrm{~cm})$, leur système est lent et nécessite un grand déploiement d'étiquettes et/ou d'antennes. Zhang et al. [28] présente une nouvelle approche qui utilise les angles d'arrivés avec la technologie RFID passive pour localiser des objets. C'est un concept très intéressant. Par contre, il a été testé que par simulation et l'estimation de l'erreur obtenue est d'environ $100 \mathrm{~cm}$. 


\subsection{Les approches basées sur la trilatération}

En dépit du nombre élevé de modèles de localisation basés sur le RFID, seul un petit nombre a exploré la méthode de trilatération. Dans les faits, cette technique est assez difficile à utiliser avec des informations bruitées et imprécises. Qing $\mathrm{Fu}$ et al. [34] ont effectué une expérience à grande échelle avec une méthode qui utilise la trilatération de manière comparable à notre nouveau modèle. Ils ont utilisé des balises actives pour localiser des piétons portant un petit lecteur. Ils pourraient atteindre une précision de 2-3 mètres ce qui est assez bon compte tenu de leur contexte expérimental. Récemment, Kim \& Kim [35] ont présenté une alternative intéressante à la technique classique. Leur modèle utilise le délai entre l'émission et la réception

du signal pour calculer la distance entre une balise active et une antenne. $\grave{A}$ partir de cette information, ils effectuent un calcul classique de trilatération leur donnant une précision au mètre près. Leur contribution est l'introduction d'une antenne à polarisation circulaire et un filtre de positionnement. Une autre approche qui vaut la peine d'être mentionnée est celle de Chen et al. [29] qui effectue une trilatération avec différentes technologies utilisant les radiofréquences (ZigBee). Ils exploitent un moteur d'inférence floue avec une variable qui corrèle le RSSI d'un transmetteur avec sa distance le séparant d'un récepteur. Ils ont atteint une précision de $119 \mathrm{~cm}$.

\subsection{Conclusion}

Comme nous l'avons susmentionné, aucune de ces approches ne peut complètement répondre précisément aux besoins des chercheurs dans le contexte d'un habitat intelligent. Il est intéressant de préciser que beaucoup de chercheurs ont exploité la technologie RFID pour reconnaître les AVQ d'une personne [38, 50-52]. La reconnaissance des activités exercées dans un habitat intelligent est actuellement le problème le plus difficile que les chercheurs doivent faire face dans l'élaboration d'une intelligence ambiante destinée aux habitats intelligents. Ce 
fait démontre bien l'intérêt et la nécessité du développement d'un système de localisation d'objets dans un environnement intelligent. Le nouveau modèle proposé dans ce mémoire surpasse la plupart des techniques de localisation existantes. De plus, il est purement basé sur la technologie RFID avec des étiquettes passives et évite, donc, les inconvénients liés aux technologies hybrides, aux balises actives alimentées par batterie et au déploiement dense de balises de référence. Par ailleurs, de nombreux systèmes, qui ont présenté des résultats comparables, ont été testés dans des conditions irréalistes [20] ou seulement par simulation [48, 53]. Le prochain chapitre décrit un nouveau modèle de multilatération précis qui est basé uniquement sur la technologie RFID passive. 


\section{CHAPITRE 3}

\section{MULTILATÉRATION AMÉLIORÉE}

\subsection{Introduction}

Dans le chapitre précédent, nous avons présenté les principales approches qui ont montré l'intérêt de l'utilisation de la trilatération ou de la multilatération pour effectuer la localisation d'étiquettes RFID passives à l'intérieur d'un habitat intelligent. Les résultats obtenus par ces différentes approches se sont avérés très intéressants. Malheureusement, aucune de celles-ci n'offre une solution complète et pratique. Par conséquent, un nouveau modèle de multilatération améliorée a été développé pour la localisation en 2D avec la technologie RFID passive. Cette nouvelle méthode de positionnement a été conçue avec l'intention de la rendre facilement utilisable sur un grand nombre de configurations matérielles de même que dans une multitude de contextes variés. Ce nouveau modèle de positionnement beaucoup plus flexible s'effectue en 3 étapes. Tout d'abord, lors de la première étape, appelée filtrage RSSI, on applique successivement les 3 filtres suivants: le filtre de réduction des fausses lectures, le filtre de réduction de crête et finalement, le filtre de pondération par moyenne gaussienne. Par la suite, on procède à la multilatération, c'est-à-dire au positionnement à proprement dit. Finalement, la troisième étape consiste à l'application du filtre de comportement du mouvement afin d'en extraire la position finale. Ce filtre introduit une correction du déplacement des objets pour permettre un positionnement, en temps réel, plus fidèle d'un objet en mouvement. Ce chapitre vise à décrire exhaustivement les différentes étapes (filtres) de cette nouvelle méthode de positionnement d'étiquettes RFID passives. Ce chapitre constitue la contribution théorique de ce mémoire. 


\subsection{Le filtrage des forces de signaux}

\subsubsection{Filtre de réduction des faux positifs et des faux négatifs}

Dans un environnement intérieur comme les habitats intelligents où les signaux sont bruités imprévisiblement, un des défis relatifs à l'utilisation d'étiquettes RFID passives pour la localisation sont les erreurs fréquentes de fausses lectures. Par exemple, une étiquette peut momentanément ne plus être captée électroniquement par les antennes alors qu'elle se situe encore à l'intérieur de la zone couverte. Par opposition, elle peut aussi être captée pendant un court instant alors qu'en réalité, elle n'y est plus. Ce type de problème se produit avec tous les systèmes RFID passifs et encore plus fréquemment avec les systèmes peu couteux. Brusey \& al. [54] ont identifié trois raisons pour expliquer cette situation :

- Le lecteur peut échouer la détection de toutes les étiquettes pendant une certaine période de temps à cause d'un problème interne inconnu.

- Les signaux émis à partir de plusieurs étiquettes peuvent entrer en collision.

- Une interférence peut se produire à cause des émissions environnantes ou à cause des matériaux métalliques à proximité.

Pour minimiser l'impact de ce problème, un filtre de réduction des faux positifs et des faux négatifs similaires à celui décrit dans [54] a été introduit au nouvel algorithme de localisation. Le filtre développé est basé sur la règle générale qu'une étiquette RFID est considérée comme absente de la zone de couverture d'une antenne seulement après un certain délai durant lequel le signal émis par l'étiquette n'ait pas été capté. Brusey \& al. [54] ont introduit une fonction qui exclut toutes les lectures dont le moment de réception est trop éloigné du moment courant. Avec cette méthode, une étiquette est considérée comme détectée aussitôt qu'il y a plus qu'une détection durant un intervalle de temps prédéfini. Le filtre développé et 
utilisé par le nouveau système de localisation est une version modifiée de cette technique. Comme le montre la figure 3.1, ce filtre itératif remplace les erreurs de faux négatif par la dernière valeur obtenue alors que les données brutes qui sont considérées comme des erreurs potentielles de faux positif sont éliminées. Donc, à la figure ci-dessous, on peut voir que le filtre continue à répéter pendant $x$ itérations la dernière valeur captée et que lorsque le signal reprend après une période de temps jugé trop longue l'algorithme attend $x$ itérations pour s'assurer que le signal soit stable à nouveau.

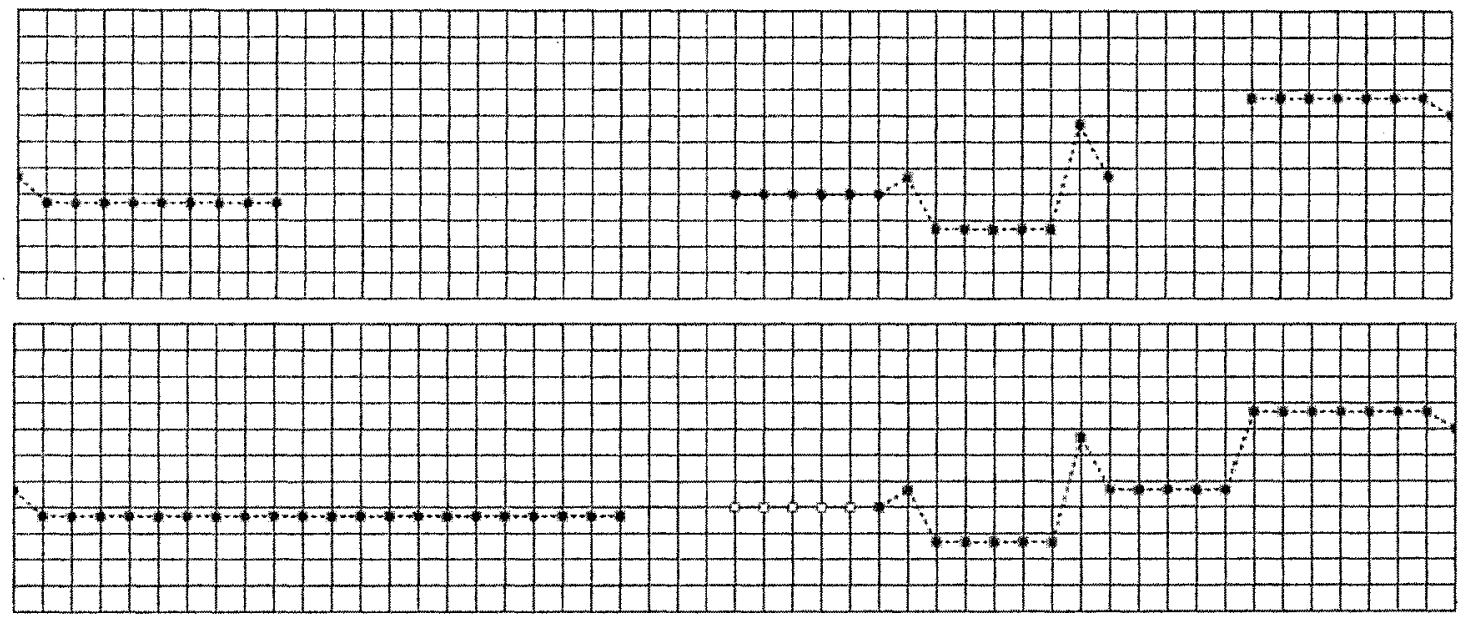

Figure 3.1: Traitement en temps réel de la force du signal d'une étiquette RFID statique. La force du signal brute est en bleu et la force du signal filtré est en rouge.

Plus précisément, à partir d'une fonction booléenne notée par $f_{i t e}(i)$ et définie en (3.1), on peut déterminer si l'état de détection d'une étiquette a changé en effectuant la soustraction de l'itération de la détection $\left(i_{d}\right)$ à l'itération courante $\left(i_{c}\right)$ et en comparant ensuite avec un certain intervalle $\Delta i$ qui représente le nombre minimum d'itérations que l'objet détecté a besoin d'être stable avant de considérer qu'il y ait eu un changement de son état (détecté ou non détecté). L'intervalle $\Delta i$ peut se déterminer en connaissant le taux de fausses lectures mais par expérience, une valeur assez élevée, disons entre 10 et 20 , s'est avérée un excellent choix. 


$$
f_{i t e}(i)=\left\{\begin{array}{lr}
\text { Vrai } & \left|i_{c}-i_{d}\right| \geq \Delta i \\
\text { Faux } & \text { sinon }
\end{array}\right.
$$

\subsubsection{Filtre de réduction de crête ou de variation anormalement élevée}

Certaines perturbations électromagnétiques peuvent provoquer une variation anormale de la force du signal RSSI. Pour pallier à ce problème, un filtre réduisant les variations trop élevées de la force du signal RSSI d'une itération à l'autre pour chaque étiquette RFID a été défini. Ce filtre est basé sur le principe que la variation de la force du signal peut être considérée comme un changement de distance par rapport à l'antenne. Ainsi, on peut, en quelque sorte, définir une vitesse de déplacement maximum en fixant une variation maximale de la force du signal pendant une itération. Ce filtre peut être utilisé pour augmenter la réactivité du système en réduisant la taille de l'échantillon utilisée par le filtre de moyenne gaussienne qui sera présenté dans la prochaine sous-section.

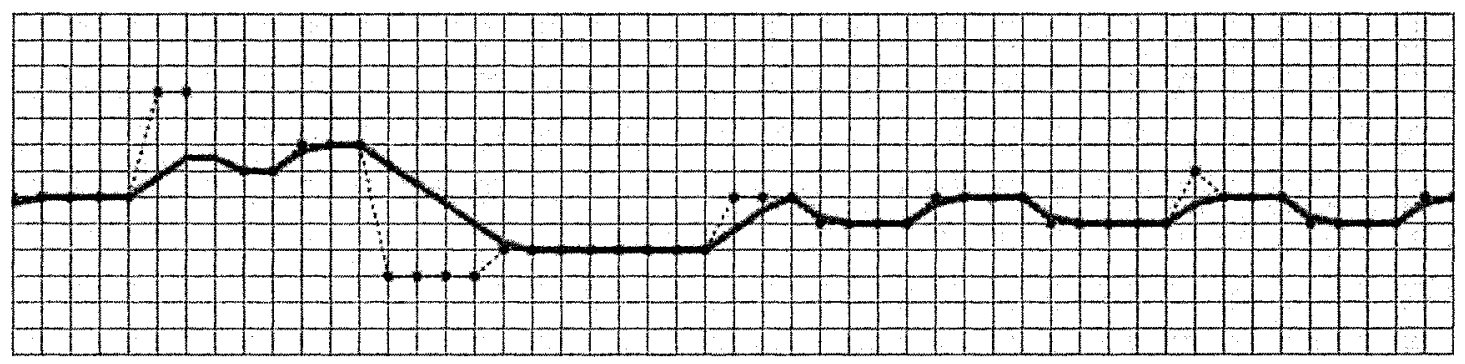

Figure 3.2 : Traitement en temps réel de la force du signal d'une étiquette RFID statique. La force du signal brute est en bleu et la force du signal filtré est en rouge.

\subsubsection{Filtre de pondération par moyenne gaussienne}

Avec les systèmes passifs, les forces de signaux (RSSI) varient souvent et significativement sans que l'objet suivi ne se soit déplacé. Ce problème a pour conséquence de créer de faux déplacements aléatoires autour de la position estimée de l'objet entre deux itérations. Il n'existe présentement aucune méthode pour éliminer complètement ce problème de nature matériel mais en appliquant une pondération par moyenne gaussienne sur les données 
reçues par les antennes, on peut considérablement réduire les variations de l'intensité des forces de signaux (RSSI). Dans l'algorithme implémenté, la courbe normale a comme moyenne (centre) le numéro de l'itération courante $\left(i_{c}\right)$ comme on peut le constater dans l'équation (3.2). Le paramètre $i$ est le numéro de l'itération associée avec la donnée RSSI qui est pondérée et la constante $\sigma$ (qui correspond normalement à l'écart type) est fixée à 2. Habituellement, la valeur de $\sigma$ est déterminée automatiquement en fonction de la vitesse de lecture du récepteur et de la période de temps sur laquelle porte la pondération. Nous avons déterminé expérimentalement, lors d'une phase d'apprentissage, que le choix $\sigma=2$ optimisait l'exactitude du positionnement. Cette phase consistait à déplacer un objet dans la cuisine du LIARA selon un trajet préétabli et ce premier déplacement devenait le trajet étalon. Par la suite, le trajet était répété et un logiciel testait un ensemble de configurations possibles (par exemple, des valeurs de $\sigma$ différentes) afin de faire correspondre le plus fidèlement possible les deux trajets. Ce processus a été répété à maintes reprises et nous a permis de déduire la valeur optimale $\sigma=2$. La valeur moyenne et pondérée du RSSI est calculée en utilisant la formule (3.3) :

$$
\begin{gathered}
f_{\text {Gaussian }}(i)=e^{-\frac{1}{2}\left(\frac{i_{c}-i}{\sigma}\right)^{2}} \\
f_{\text {strenght }}\left(t\left[i_{c}\right]\right)=\frac{\sum_{i=i_{c}-\Delta i}^{i_{c}} t[i]_{r s s i} f_{\text {Gaussian }}(i)}{\sum_{i=\Delta i}^{i_{c}} f_{\text {Gaussian }}(i)}
\end{gathered}
$$

où $t[i]_{r s s i} * f_{\text {Gaussian }}(i)$ représente le RSSI pondéré pour la $i^{i e ̀ m e}$ itération. Cette fonction reçoit comme paramètre le RSSI d'une étiquette pour calculer la valeur correspondante au poids moyen. Le paramètre $(\mathrm{t}[])$ consiste en un tableau contenant les RSSI pour chaque itération. Ensuite, la somme pondérée des forces de signaux, satisfaisant la contrainte $i_{c}-i \leq \Delta i$, est divisée par le poids total des lectures. La constante $\Delta i$ est le nombre d'itérations considérées par 
le filtre de pondération par moyenne gaussienne dont on peut apercevoir un exemple de traitement à la figure 3.3 .

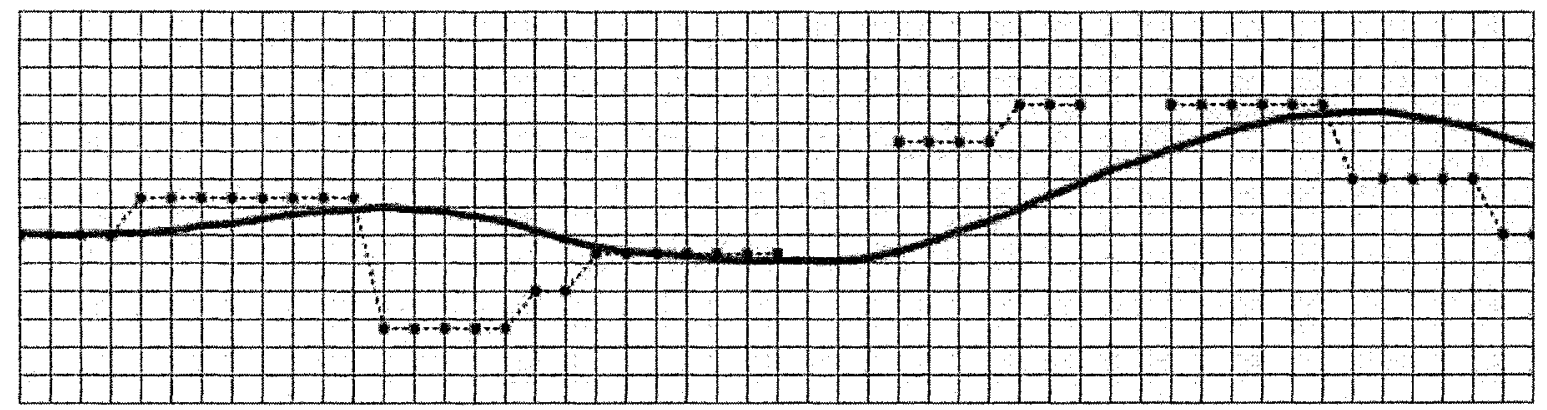

Figure 3.3 : Traitement en temps réel de la force du signal d'une étiquette RFID statique. La force du signal brute est en bleu et la force du signal filtré est en rouge.

\subsection{La multilatération elliptique}

\subsubsection{Multilatération par intersection géométrique de disques elliptiques}

Une multilatération est l'estimation de la coordonnée d'un objet cible à partir de la distance entre cette cible et plusieurs positions de référence. Cependant, un des problèmes avec l'utilisation d'une multilatération est que la plupart des antennes RFID sont directionnelles. Donc, une figure géométrique circulaire ne correspond pas au modèle de propagation des ondes émises par les antennes. Par contre, une figure elliptique y correspond beaucoup plus fidèlement. De ce fait, l'algorithme développé utilise les ellipses pour répondre à cette problématique. Par conséquent, l'algorithme est donc plus général puisqu'un cercle est un cas particulier d'une ellipse dont le grand et le petit axe seraient égaux. Ainsi, notre méthode est applicable dans les cas classiques (circulaires) de même que dans les cas spéciaux où les antennes utilisées ne sont pas omnidirectionnelles rendant ainsi notre modèle de positionnement utilisable dans une multitude de contextes variés.

Pour être en mesure d'effectuer une multilatération elliptique, il faut, tout d'abord, modéliser mathématiquement la déperdition de la force du signal RSSI en fonction de la distance 
et ce de façon parallèle et perpendiculaire à l'antenne. Cette modélisation nous permet de calculer, par la suite, le demi grand axe et le demi petit axe de l'ellipse en fonction de la force du signal reçu par l'antenne. Puisqu'à chaque positionnement, les quatre antennes de notre système mesurent une force de signal et produisent donc une ellipse de positions possibles, il devrait y avoir normalement 4 modélisations (une par antenne) mathématiques de ce type. Dans notre cas, les quatre antennes se comportent de la même manière. En mesurant, successivement la force du signal reçu par l'antenne en fonction du déplacement vertical (demi petit axe) et horizontal (demi grand axe) de l'étiquette RFID par rapport à l'antenne, nous avons obtenu, en utilisant la régression polynomiale de degré deux, les équations nous permettant de calculer le demi grand axe et le demi petit axe de l'ellipse en fonction de la force du signal reçu. Explicitement, nous avons obtenu, pour notre système, les relations suivantes :

$$
\begin{aligned}
& \text { DemiGrand }_{a x e}(R S S I)=0.1833 \cdot R S S I^{2}+8.5109 \cdot R S S I+104.3 \\
& \text { DemiPetit }_{a x e}(R S S I)=0.0462 \cdot R S S I^{2}+0.8155 \cdot R S S I+104.3
\end{aligned}
$$

où les coefficients de corrélation obtenus par la méthode de régression polynomiale sont de 0.974 pour le grand axe $\left(R_{G r a n d}^{2}=0.974\right)$ et de 0.937 pour le petit axe $\left(R_{P e t i t}^{2} t_{a x e}=0.937\right)$.

Nous pouvons, désormais, à partir d'une force de signal reçu par une antenne (RSSI), calculer l'équation de l'ellipse sur laquelle l'objet devrait normalement se situer. Celle-ci se calcule à l'aide de la formule suivante :

$$
\frac{\left(x-x_{c 1}\right)^{2}}{\text { DemiGrand }_{\text {axe }}(R S S I)^{2}}+\frac{\left(y-y_{c 1}\right)^{2}}{\text { DemiPetit }_{\text {axe }}(R S S I)^{2}}=1
$$

où $x_{c 1}$ représente la coordonnée de l'antenne 1 selon l'axe des $\mathrm{x}$ et $y_{c 1}$ celle de l'antenne 1 selon l'axe des y. 
L'étape subséquente consiste à trouver le point d'intersection entre toutes les ellipses formées par la mesure de la force du signal mesurée. Cependant, il n'y a pratiquement jamais qu'un seùl point d'intersection dans un contexte réel. Pour résoudre ce problème, le concept de zone commune a été introduit. L'idée théorique derrière ce concept d'intersection géométrique est qu'une étiquette qui est captée se situe sur le disque elliptique dont les dimensions sont obtenues à partir des RSSI reçus et dont l'orientation est celle de l'antenne correspondante. Donc, par déduction, l'étiquette devrait se situer dans la zone d'intersection entre toutes les ellipses formées autour des antennes l'ayant captée. Ensuite, la position estimée est le barycentre de cette zone commune tel qu'illustré à la figure 3.4. Veuillez noter que la figure 3.4 représente un cas réel de positionnement avec du matériel physique (antennes et étiquettes RFID passives), que l'algorithme a été mis à l'épreuve par simulation et que lorsqu'il n'y a pas erreur de force de signal la position calculée est la même que la position de l'objet.

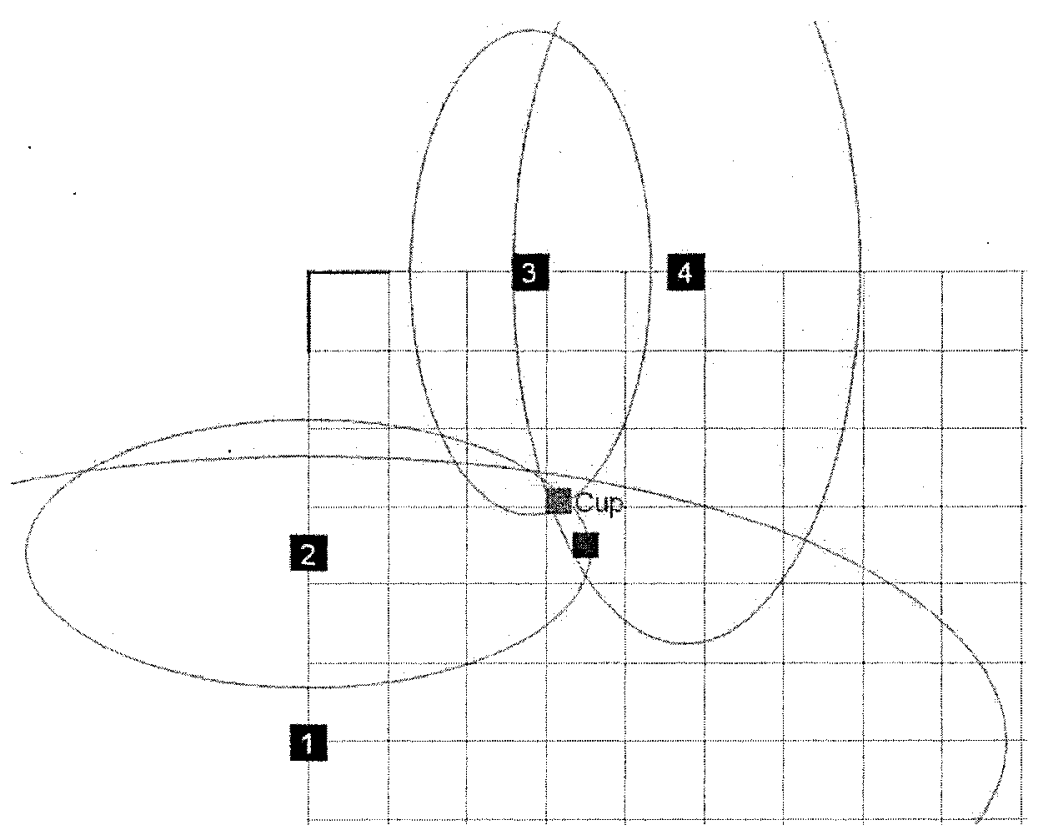

Figure 3.4 : Positionnement en temps réel d'un objet. Les ellipses vertes sont calculées à partir des RSSI, le carré vert est la position calculée par l'algorithme et le carré rouge est la position réelle de l'objet. 


\subsubsection{Recherche ou raffinement de la solution}

Les erreurs de RSSI peuvent faire en sorte que les ellipses n'ont pas d'intersection ou que la zone commune soit trop grande. Dans ces cas, les dimensions des ellipses sont automatiquement ajustées de manière inversement proportionnelle à la force du signal (plus la force du signal est grande plus les dimensions de l'ellipse sont précises et moins elles sont modifiées). Ce phénomène est clairement représenté par les deux exemples contenus dans la figure 3.5.

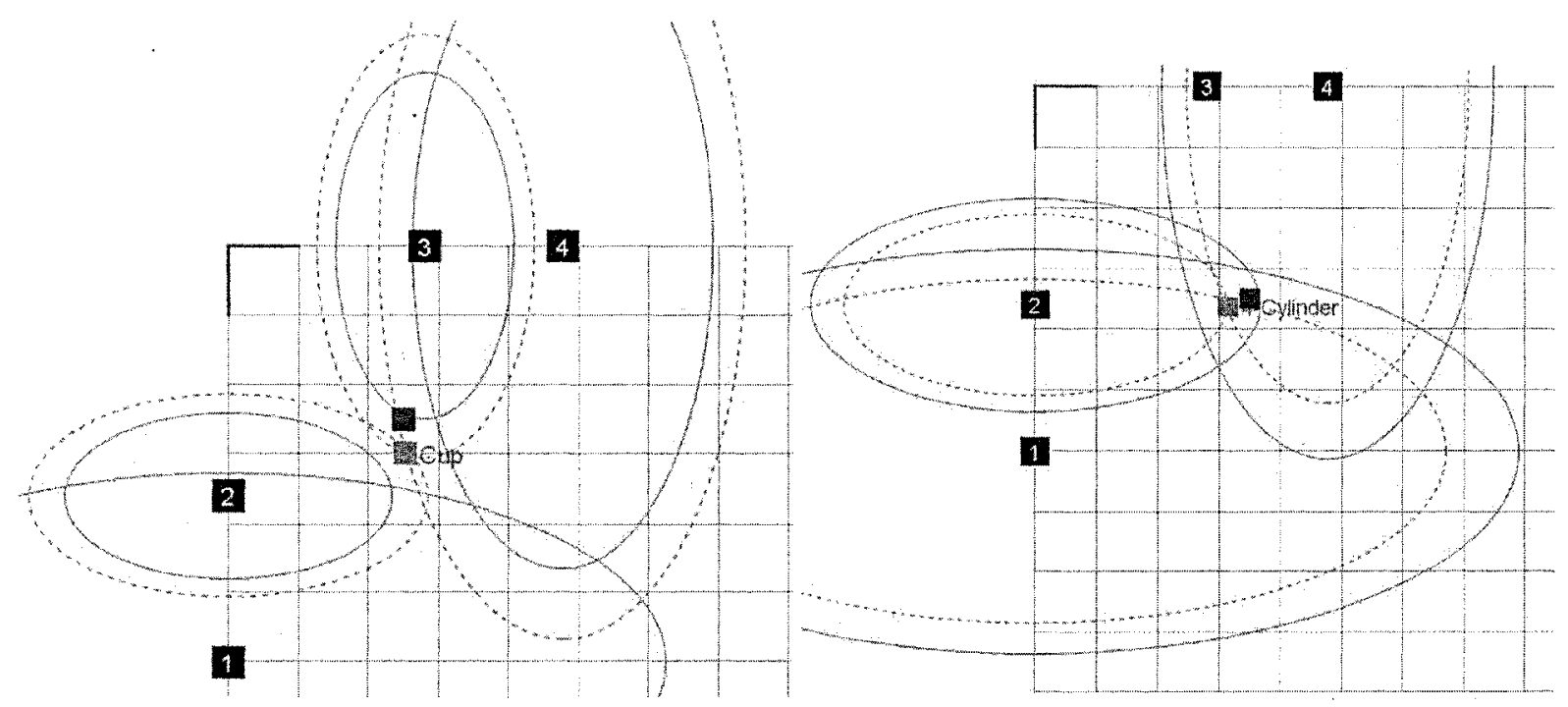

Figure 3.5 : Positionnement en temps réel d'un objet. Les ellipses vertes sont calculées à partir des RSSI et les ellipses en pointillées sont celles corrigées par l'algorithme de positionnement. Le carré vert est la position estimée par l'algorithme de positionnement tandis que le carré rouge est la position réelle de l'objet.

\subsection{Règles définissant le comportement du mouvement des objets}

Comme nous l'avons mentionné précédemment, les perturbations électromagnétiques peuvent causer des variations anormales de la force du signal et, donc, causer un faux déplacement. Pour remédier à ce problème, des règles qui définissent le comportement du mouvement des objets ont été définies. Ces règles font en sorte que l'objet bouge correctement d'un point de vue de la physique en considérant certains paramètres comme la vitesse, 
l'accélération et la masse de l'objet. Cet algorithme permet aussi de définir la vitesse maximale à laquelle l'objet est susceptible de se déplacer et prend en considération la vitesse ainsi que l'orientation du déplacement de l'objet pour s'assurer que sa direction soit stabilisée au fil du temps. Cet algorithme pourrait être considéré comme un filtre de positions qui est inspiré des comportements de déplacement et de direction utilisés pour apporter un mouvement plus naturel aux entités dans les jeux vidéo et dans la simulation 3D [55].

\subsubsection{La position, la vitesse et le mouvement}

La mise en œuvre de toutes les forces impliquées dans un comportement de mouvement ou de direction peut être représentée par des vecteurs mathématiques. La figure 3.6, représente un objet positionné en $(x, y)$ avec une vitesse représentée par le vecteur $\overrightarrow{(a, b)}$. Tout d'abord, la vitesse de l'objet est obtenue selon la formule suivante :

$$
\overrightarrow{\text { Vitesse }}=\text { normaliser }(\overrightarrow{\text { Clble }}-\overrightarrow{\text { Position }}) * \text { vitesse_maximale }
$$

où le vecteur cible représente la nouvelle position calculée par l'algorithme de localisation et où la fonction normaliser consiste à ramener le vecteur à une norme de 1 en le multipliant par un scalaire égal à l'inverse de sa norme (longueur). L'orientation du vecteur de vitesse représente la direction vers laquelle l'objet se dirige tandis que sa norme détermine son déplacement au cours d'une itération. Par ailleurs, la normalisation du vecteur suivi d'une multiplication par un scalaire a pour effet de le redimensionner à la vitesse maximale. Le mouvement et la nouvelle position sont ensuite calculés en utilisant la formule :

$$
\overrightarrow{\text { Nouvelle_position }}=\overrightarrow{\text { Position }}+\overrightarrow{\text { Vitesse }}
$$




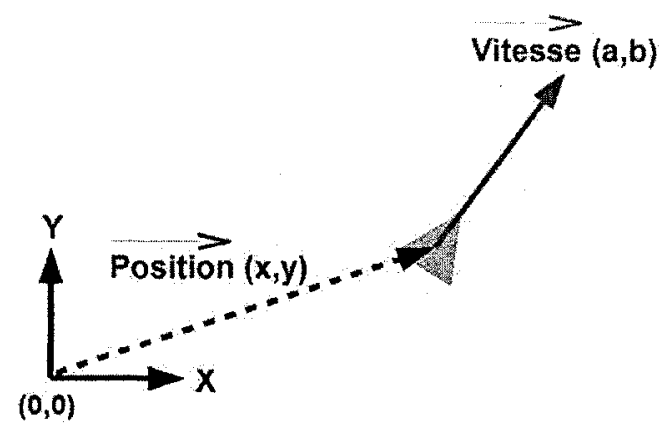

Figure 3.6 : Position et vitesse

Jusqu'ici, l'algorithme effectue simplement un redimensionnement du déplacement à la vitesse maximale. L'idée derrière ce filtre ou plutôt derrière ces règles régissant le comportement de mouvement des objets est d'influencer leur mouvement en ajoutant des forces pour rendre leurs déplacements plus naturels ce qui a pour effet de lisser leurs trajets par la même occasion. Pour ce faire, une force de direction est ajoutée aux deux autres forces. L'addition des forces régissant le comportement du mouvement permet à l'objet d'ajuster en douceur sa vitesse et d'éviter les changements brusques de trajectoire. Si la cible se déplace, l'objet va progressivement modifier son vecteur vitesse pour essayer d'atteindre cette nouvelle position calculée par multilatération elliptique sans nécessairement avoir le temps de s'y rendre. Le comportement du mouvement comporte deux forces : la vitesse désirée et la force de direction. Le vecteur représentant la vitesse désirée est une force qui dirige le déplacement de l'objet vers la position cible. Le vecteur force de direction est le résultat de la soustraction de la vitesse désirée par la vitesse courante et il provoque un changement progressif de la direction du déplacement vers la cible. De plus, la force de direction est divisée par la masse de l'objet ce qui produit des vitesses de déplacement différentes et, donc, des comportements du mouvement personnalisés aux différents types d'objets selon leur poids. La figure 3.7 illustre un exemple des différentes forces à la base du comportement de mouvement qui sont calculées avec les formules suivantes : 


$$
\begin{aligned}
& \overrightarrow{\text { Vitesse_désırée }}=\text { normaliser }(\overrightarrow{c l b l e}-\overrightarrow{\text { Positıon }}) * \text { vitesse_maximale } \\
& \overrightarrow{\text { Force_dırectıonelle }}=(\overrightarrow{\text { Vltesse_désırée }}-\overrightarrow{\text { Vitesse }}) / \text { masse }
\end{aligned}
$$

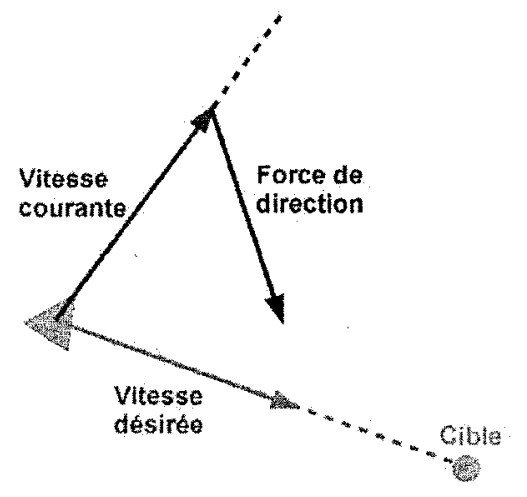

Figure 3.7 : Forces régissant le comportement de mowvement

Après que la force de direction ait été calculée, elle doit être ajoutée au vecteur représentant la vitesse courante. L'addition de la force de direction à chaque itération fera abandonner peu à peu sa direction précédente pour aller graduellement vers la nouvelle position cible. La nouvelle position est, finalement, obtenue à l'aide des formules suivantes :

$$
\begin{gathered}
\overrightarrow{\text { Vitesse }}=\text { tronquer }(\overrightarrow{\text { Vitesse }}+\overrightarrow{\text { Force de direction_ajustée, }}, \text { vitesse_maximale }) \\
\qquad \overrightarrow{\text { Nouvelle_positıon }}=\overrightarrow{\text { Positıon }}+\overrightarrow{\text { Vitesse }}
\end{gathered}
$$

où la fonction tronquer(vecteur, scalaire) consiste à réduire la norme d'un vecteur à une certaine longueur qui est égal à un scalaire. La transition fluide du mouvement est calculée en effectuant l'addition de la force de direction à la vitesse de l'objet pour obtenir le nouveau vecteur vitesse qui prend un certain temps pour changer et pointer vers la nouvelle cible. En conclusion, ce comportement du mouvement produit un trajet similaire à celui représenté en orange à la figure 3.8 . 


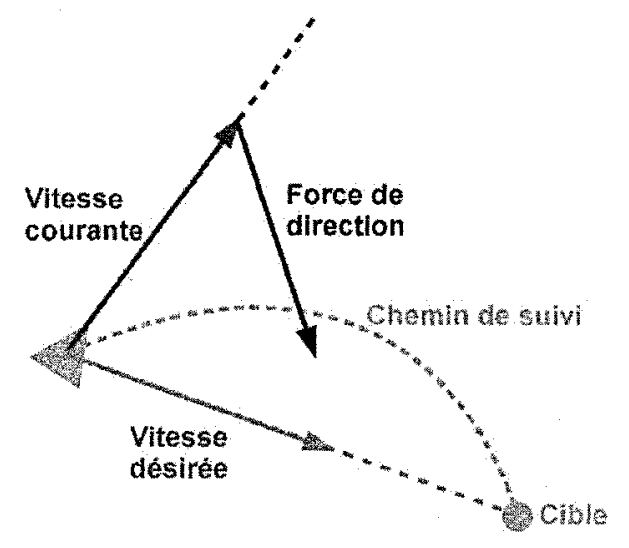

Figure 3.8: Trajectoire de déplacement

\subsubsection{La zone de ralentissement}

Maintenant que l'objet se dirige correctement vers sa cible, on doit définir son comportement lorsqu'il est sur le point d'atteindre la destination. Comme on peut le voir à la figure 3.9, le comportement d'arrivée vers la cible est simple. L'objet se déplace à sa vitesse maximale lorsqu'il n'est pas à proximité de la cible et il ralentit graduellement à l'approche de sa destination pour éventuellement s'y arrêter. Son ralentissement est calculé avec la formule suivante :

$\overrightarrow{\text { Vitesse }}=$ tronquer $(\overrightarrow{\text { Vitesse }}$, vitesse_maximale $) * \frac{\text { longueur }\left(\overrightarrow{P_{0} \text { itition }}-\overrightarrow{\text { Cible }}\right)}{\text { rayon_zone_ralentissement }}$

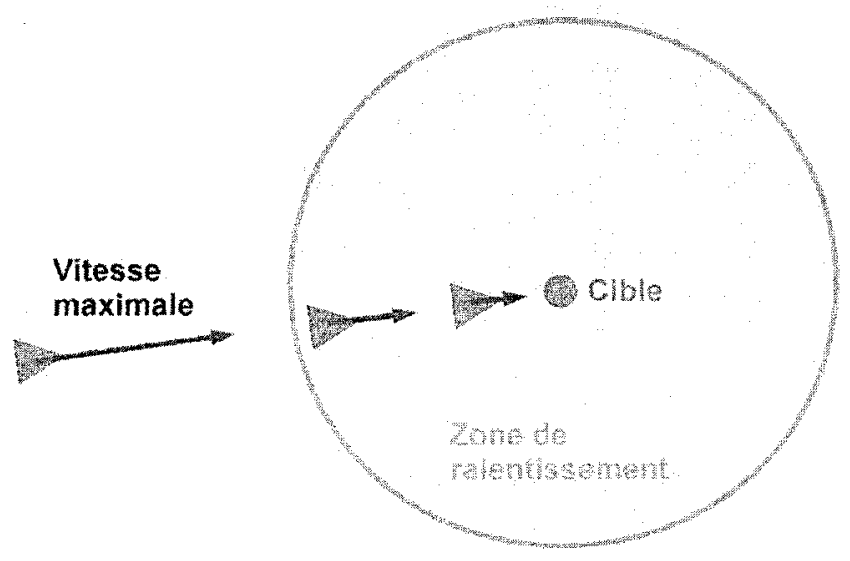

Figure 3.9 : Comportement d'arrivé vers la cible 


\subsubsection{Bilan sur l'approche}

L'idée derrière ce nouveau filtre est de produire des résultats complexes pour rendre les déplacements des objets plus naturels tout en effectuant des calculs vectoriels simples basés sur trois vecteurs représentant des forces : la vitesse, la force de direction et la vitesse désirée. Pour conclure, tel que montré à la figure 3.10 , on peut calculer un trajet naturel de suivi en utilisant ces trois vecteurs et la méthode suivante :

1. Déterminer la vitesse/direction désirée en calculant le vecteur normalisé entre la position actuelle et la position cible.

2. Calculer la distance à parcourir.

3. Si la distance n'est pas plus petite que le rayon de la zone de ralentissement alors le déplacement est effectué à la vitesse maximale.

4. Si l'on est à l'intérieur de la zone de ralentissement alors on commence à ralentir pour arrêter en douceur sur la position cible avec une décélération progressive.

5. Calculer la force de changement de direction en soustrayant la vitesse actuelle à la vitesse désirée.

6. Ajout du vecteur représentant la force de direction au vecteur de la vitesse courante pour, ensuite, calculer la nouvelle position.

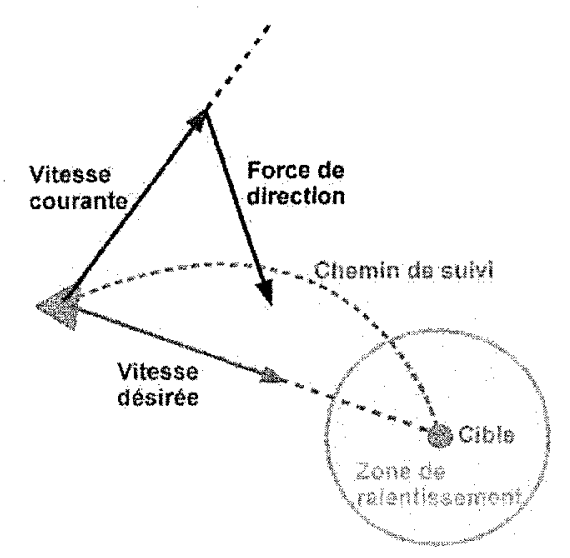

Figure 3.10: Comportement de mouvement 


\subsection{Conclusion}

Ce chapitre avait pour but de présenter une nouvelle approche pour la localisation d'étiquettes RFID passives à l'intérieur de l'habitat intelligent. Notre approche se compose de trois étapes majeures qui sont résumées schématiquement à la figure 3.11. Ces trois étapes sont répétées à chaque itération.

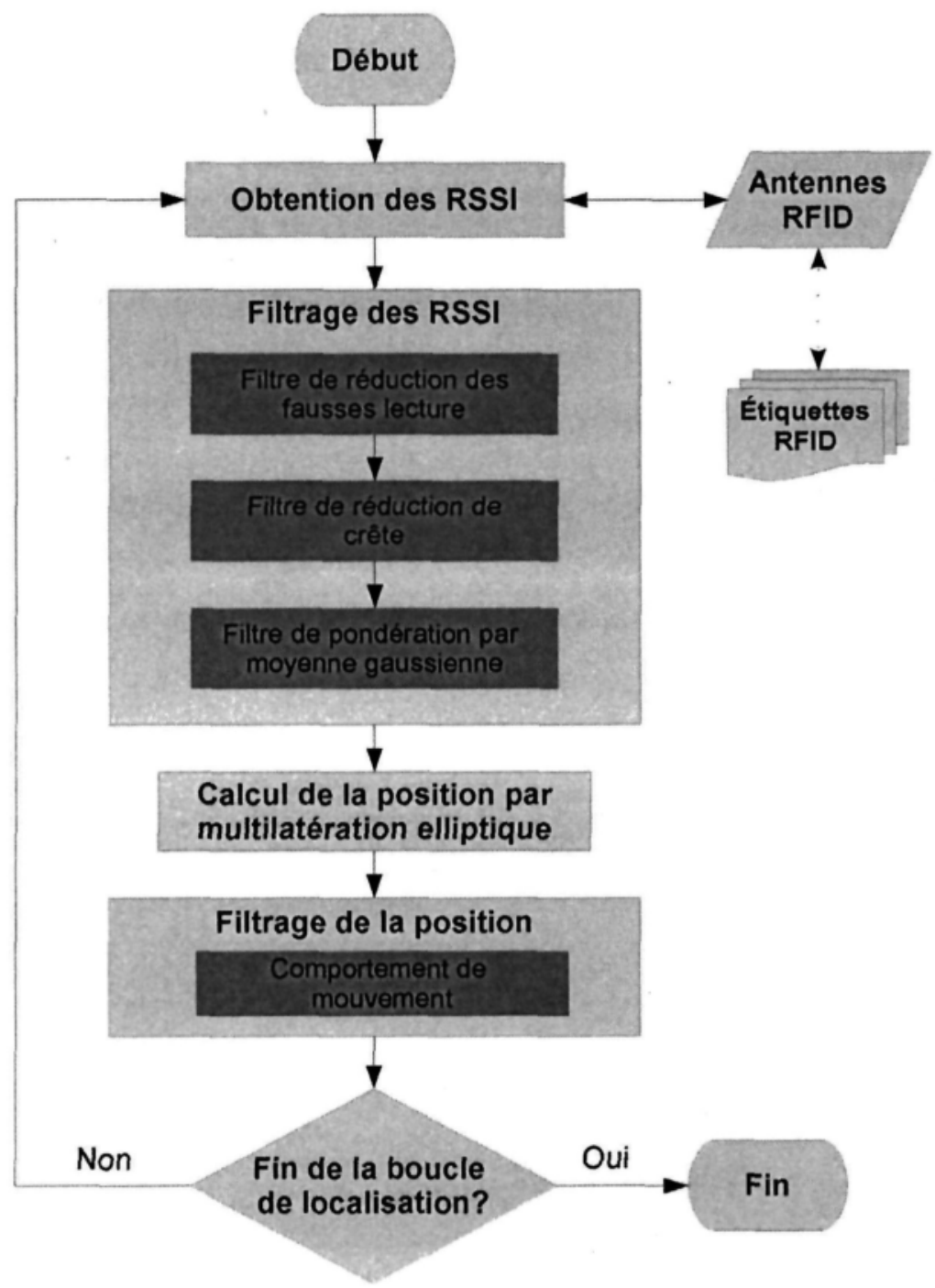

Figure 3.11 : Déroulement du processus de localisation

La première étape est celle du filtrage des RSSI envoyés par les étiquettes et reçus par les antennes RFID. Cette étape commence par la réception des forces de signaux brutes. Ensuite, les 
valeurs sont soumises à un premier filtre qui consiste à corriger les erreurs de faux positifs et de faux négatifs. Ensuite, les résultats sont envoyés au second filtre qui réduit les variations trop élevées. Finalement, les données traitées sont passées au dernier filtre qui effectue une pondération par moyenne gaussienne.

La deuxième étape consiste à transformer les RSSI en distance pour obtenir des ellipses positionnées et orientées dans la direction de l'antenne correspondante. Par la suite, le calcul de multilatération par intersection d'ellipses est effectué et les ellipses sont automatiquement ajustées de manière inversement proportionnelle à la force du signal capté dans le cas où il n'y a pas d'intersection ou que la zone commune soit considérée comme trop grande.

La troisième et dernière étape a pour but de rendre les déplacements de l'objet plus naturels d'un point de vue physique en évitant de bondir directement sur les nouvelles positions calculées par l'algorithme de multilatération. Cette étape définissant le comportement du mouvement des objets peut être considérée comme un filtre appliqué aux positions dans le sens que l'objet se dirige vers la nouvelle position sans nécessairement s'y rendre dans le cas où le déplacement désiré soit trop grand (un déplacement trop élevé lors d'une seule itération est susceptible d'être une erreur). De plus, l'effet secondaire et positif du comportement de ralentissement à l'approche de sa destination réduit les changements de position dans le cas où l'objet ne bouge pas réellement et qu'il y a des fluctuations de la force du signal dues à des interférences ou à toute sorte de phénomènes physiques.

Le chapitre suivant présente l'implémentation de la méthode de positionnement décrite et expose les résultats des expérimentations qui ont pour but de valider cette nouvelle approche de positionnement dans le contexte d'un habitat intelligent. 


\section{CHAPITRE 4}

\section{IMPLÉMENTATION ET VALIDATIONS}

\subsection{Introduction}

Dans le chapitre précédent, nous avons décrit le modèle théorique proposé dans ce mémoire permettant la mise en place de notre nouveau système de positionnement d'étiquette RFID passive. Le présent chapitre vise, maintenant, à décrire les efforts d'implémentation et d'expérimentations pratiques que nous avons effectués afin de valider le potentiel de la méthode proposée. Tout d'abord, nous débuterons par une présentation de l'implémentation du logiciel de positionnement. Nous décrirons au passage le contexte dans lequel nous avons mis en place ce nouveau système. Les détails concernant l'implémentation du logiciel de localisation seront illustrés grâce à des schémas, des illustrations, ainsi que des copies d'écrans de l'interface usager. La dernière portion du chapitre traitera des expérimentations qui ont été réalisées avec le système. Les résultats de ces expérimentations seront présentés, analysés et comparés à ceux des travaux antérieurs. Au terme du chapitre, le lecteur aura une idée claire du potentiel applicatif du nouveau système.

\subsection{Implémentation dans le laboratoire du LIARA}

Cette section décrit le contexte dans lequel le nouveau système de positionnement a été implémenté. Elle débute par une description de l'infrastructure de l'habitat intelligent. Le matériel utilisé par le système de positionnement sera présenté un peu plus en détail. Aussi, le logiciel qui a été développé pour tester l'algorithme ainsi que sa mise en œuvre sera décrit. 


\subsubsection{L'infrastructure du LIARA}

Le laboratoire LIARA dispose d'une infrastructure prototype d'envergure, financée par la Fondation Canadienne pour l'Innovation ( $\mathrm{FCI}$ ), permettant le développement de technologies d'assistance basées sur la domotique. Le prototype d'habitat intelligent est construit sur 100 mètres carrés à l'intérieur du pavillon principal de l'Université du Québec à Chicoutimi (UQAC). Il est doté d'un ensemble de capteurs (tactiles, pression, infrarouge, luminosité, RFID, ultrasons, incendient, etc.) et d'effecteurs (contrôle vocal, vidéo, son, domotique du système d'éclairage, etc.). La figure 4.1 ci-dessous montre différents éléments de l'habitat intelligent.

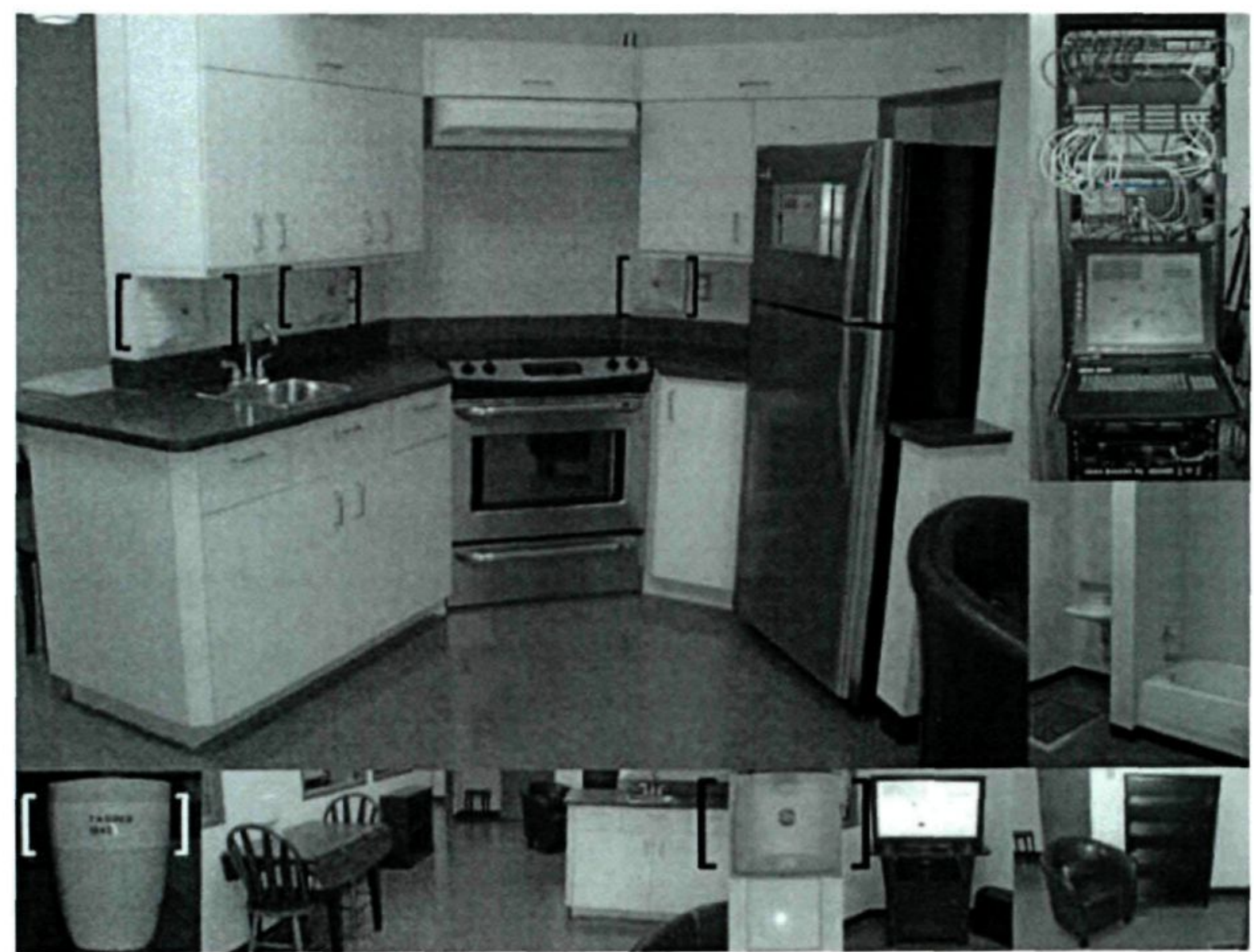

Figure 4.1: Images de l'habitat intelligent du LIARA

Sur l'image, on peut voir la cuisine où les activités les plus complexes sont effectuées et, donc, l'endroit de prédilection pour les diverses expérimentations effectuées. Sur cette image, on 
peut aussi voir, en bas à gauche, une tasse sur laquelle nous avons installé une étiquette RFID comme la plupart des autres objets (assiettes, verres, bols, etc.) pouvant être utilisé lors des activités de la vie quotidienne (préparation de repas). La télévision, en bas à droite de la figure 4.1, ainsi que l'iPAD installé sur le réfrigérateur sont des exemples d'effecteur qui peuvent être contrôlés à distance. En haut à droite, nous pouvons voir le serveur Dell qui se charge de récupérer les données de tous les capteurs ou des antennes RFID, de les traiter et de mettre à jour la basse de données contenant les informations sur ces capteurs. Les autres images montrent la salle à manger, les toilettes et la bibliothèque. Les choix de capteurs et leur installation ont été effectués afin de permettre une reconnaissance d'activités basée sur les objets de manière non intrusive et à l'insu du patient pour instaurer une atmosphère de résidence réelle plutôt que celui d'un laboratoire.

\subsubsection{Le matériel RFID utilisé}

Concernant la technologie RFID utilisé, le laboratoire possède 8 antennes RFID dans son habitat intelligent pour permettre le suivi des objets. Ces antennes couvrent les bandes radio utilisées aux États-Unis et en Europe pour la technologie RFID (860-960 MHz). Parmi ces 8 antennes, quatre antennes A-PATCH-0025 de la compagnie Poynting sont installées sur les murs (voir la figure 4.1) de la cuisine, qui correspond à l'espace expérimental utilisé par le système positionnement. Ces antennes sont polarisées circulairement pour permettre une meilleure couverture dans un environnement intérieur. De plus, l'application du contrôleur (driver) est à code source ouvert (open source) et est fournie par la compagnie. Donc, on a la possibilité de modifier et d'ajuster le système tel que désiré. Le matériel RFID a été choisi pour toutes ces raisons qui le rendent adapté au problème de la localisation d'étiquette RFID passive dans un habitat intelligent. 
Les étiquettes RFID passives utilisés à l'intérieur du laboratoire ont eu aussi été sélectionnées en fonction de différents critères comme la taille, leur solidité et la bande de fréquence utilisée (UHF). Celles qui ont finalement été sélectionnées sont les étiquettes de moyenne dimension, suffisamment solide pour un usage intensif, suffisamment grandes pour bien capter les ondes transmises par les antennes, et suffisamment petites pour être intégrées à tous types d'objets. Avec la technologie RFID passive, même si les étiquettes sont techniquement identiques, parfois leur sensibilité est très différente. Cela peut conduire à des comportements imprévisibles. Donc, avant de les installer sur les objets, on doit effectuer des tests préliminaires afin de déterminer la similarité au niveau de sensibilité des différentes étiquettes. On sélectionne ensuite avec soin celles qui ont à peu près la même sensibilité, de façon à ne pas biaiser les expérimentations.

Comme on peut le voir à la figure 4.2 , les étiquettes sélectionnées ont été intégrées à une vaste gamme d'objets de l'habitat. Les poêlons, les assiettes, les tasses, les verres, les ustensiles, et l'ensemble des objets pertinents ont été munis de ces étiquettes. La présence des antennes dans la portion cuisine de l'habitat permet alors de mettre en cuvre et de déployer le système de localisation pour effectuer le suivi, en temps réel, de tous ces objets. L'objectif visé, tel que mentionné en introduction du mémoire, est d'utiliser le service de localisation des objets comme données d'entrée pour la reconnaissance des activités de la vie quotidienne. Ainsi, l'obtention d'un certain niveau de précision est requise si l'on souhaite obtenir des résultats utiles pour la reconnaissance. Par ailleurs, plus la précision sera grande, plus l'information aura de la valeur d'un point de vue inférenciel. 


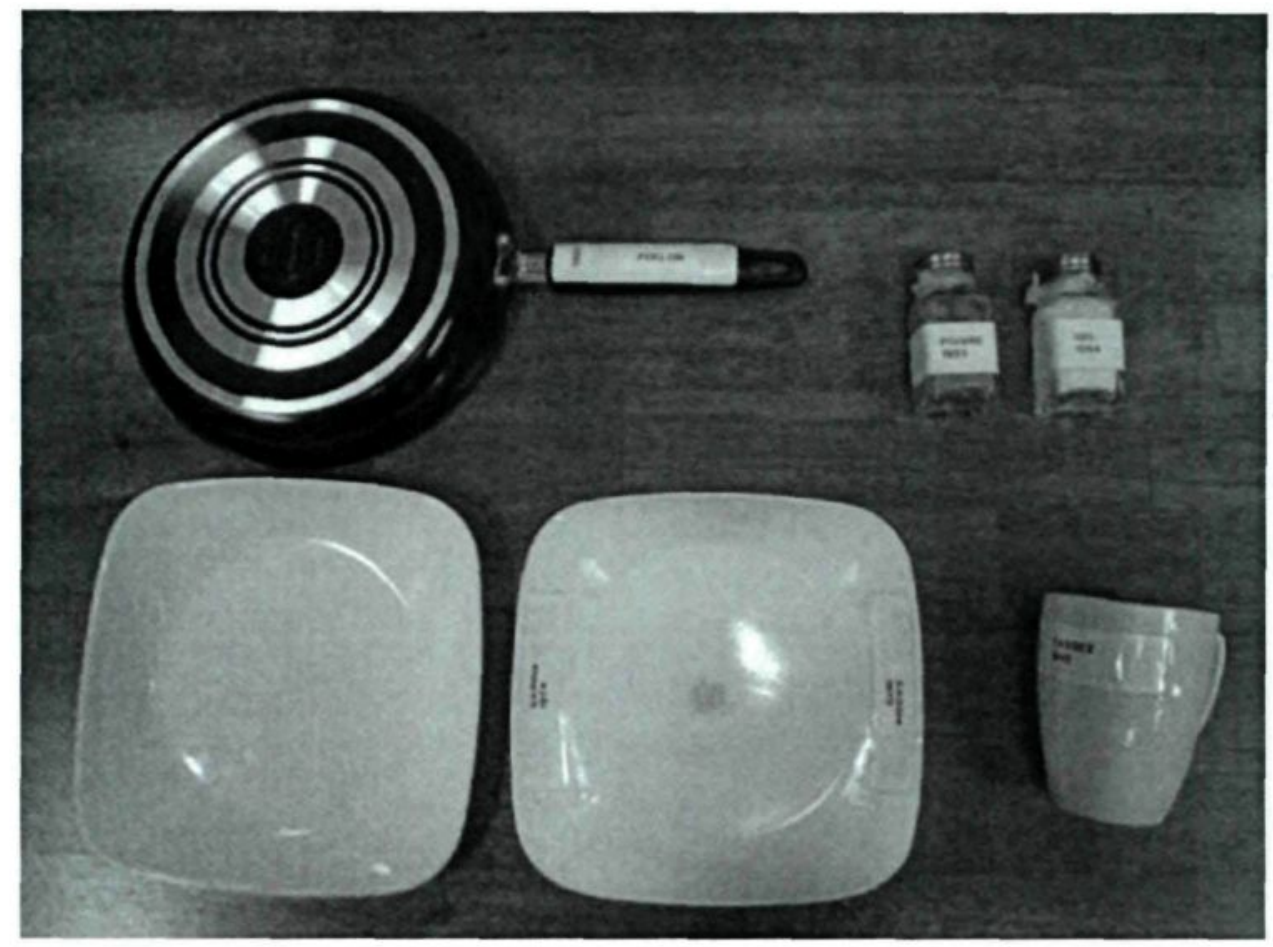

Figure 4.2: Objets munis d'étiquettes RFID passives

\subsubsection{Implémentation du système de positionnement}

L'implémentation de notre algorithme a été effectuée dans le langage orienté objet (Java). Il s'agit du langage utilisé pour la majorité des applications du laboratoire. Il permet le développement et le déploiement sur une multitude de plateformes différentes.

La figure 4.3 ci-dessous illustre la boucle de fonctionnement du système de localisation, tel qu'il a été implémenté. Tout d'abord, le logiciel communique avec le serveur de base de données SQLServer de l'habitat intelligent pour obtenir les informations en temps réel sur les étiquettes RFID passives. Ensuite, un filtre est appliqué sur les données obtenues avant qu'il soit traité par l'algorithme de positionnement. Pour finir, la position calculée par notre approche de multilatération elliptique est corrigée par un filtre qui définit les règles du comportement de mouvement de l'objet suivi avant d'envoyer ces nouvelles informations au module de reconnaissance de comportements erronés, ainsi que de mettre à jour l'interface utilisateur. 


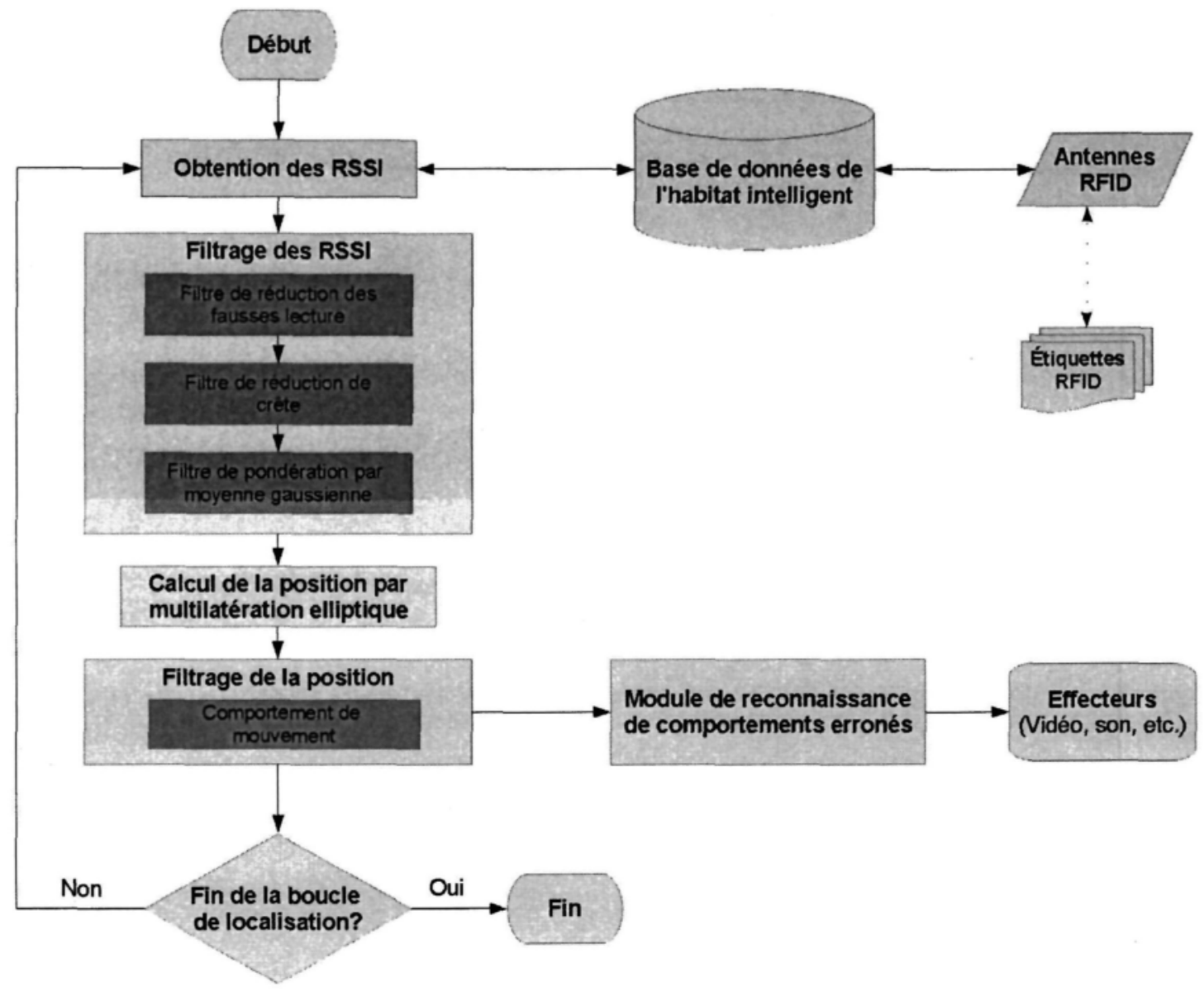

Figure 4.3 : Schéma du système

\subsubsection{La récupération des données RSSI}

Le système RFID passif fonctionne comme suit. Tout d'abord, les antennes émettent un signal dans la pièce. Deuxièmement, les balises situées à portée de l'antenne absorbent l'onde qui lui donne assez d'énergie (l'étiquette passive n'a pas de source d'alimentation interne) pour émettre un signal qui contient son identifiant unique (ID). Troisièmement, l'antenne reçoit en retour cette identification avec l'indicateur de la force du signal reçu (RSSI). Quatrièmement, le routeur traite les informations et les transmet au serveur (Windows Server 2003) qui les inscrit à une base de données à toutes les 100 millisecondes. Enfin, l'application de localisation récupère ces informations afin d'avoir les informations nécessaires dans le but de positionner les objets étiquetés avec des puces RFID passives. 


\subsubsection{L'interface utilisateur}

Le logiciel développé est constitué d'une interface utilisateur réalisée à partir de la bibliothèque Java Swing qui peut être vue aux figures 4.4 et 4.5. L'interface utilisateur comporte divers outils nécessaires à la facilitation du travail des chercheurs (affichage des ellipses obtenues pour aider à l'élaboration de l'algorithme, affichage des informations interne de l'algorithme de multilatération pour faciliter le débogage, l'ajustement et l'amélioration, graphique du traitement en temps réel des RSSI, calibration semi-automatique des paramètres, outil de simulation $2 \mathrm{D}$ et $3 \mathrm{D}$, etc.).

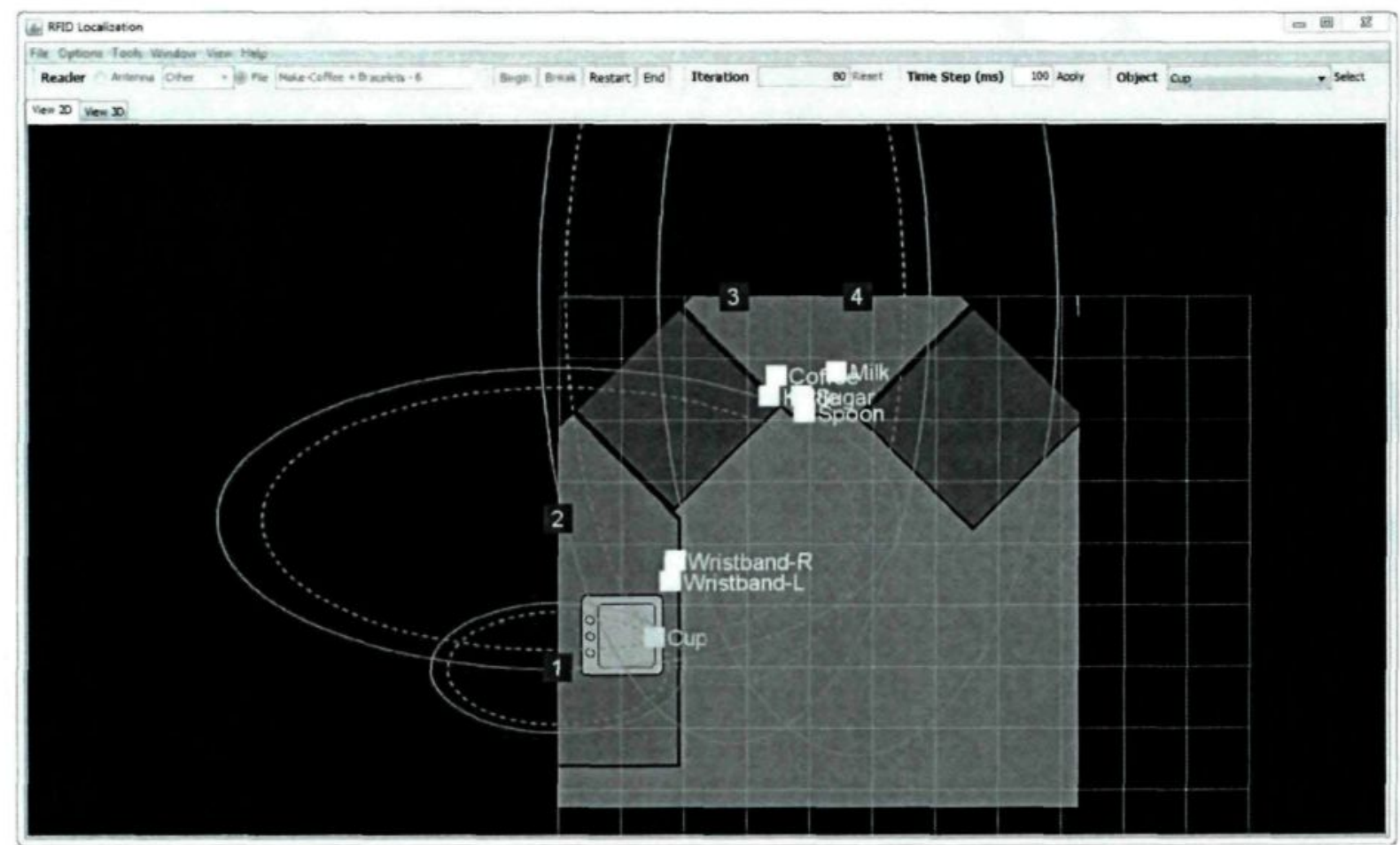

Figure 4.4: Interface utilisateur avec la vue $2 D$

En pratique, l'application de positionnement est entièrement en 3D et l'algorithme décrit au chapitre précédent effectue un positionnement en 2D sur un plan en trois dimensions (le plan passant par les positions des quatre antennes utilisées). Par ailleurs, une version en 3D de l'affichage a été implémentée (voir la figure 4.5) ainsi qu'un algorithme de multilatération 3D 
par intersection de volumes ellipsoïdaux, qui est en quelque sorte une extension du modèle d'intersection géométrique en trois dimensions. Cependant, il a été seulement validé avec l'outil de simulation incorporé au programme puisque la disposition ainsi que le nombre d'antennes de l'habitat intelligent ne permettent pas une localisation en trois dimensions.

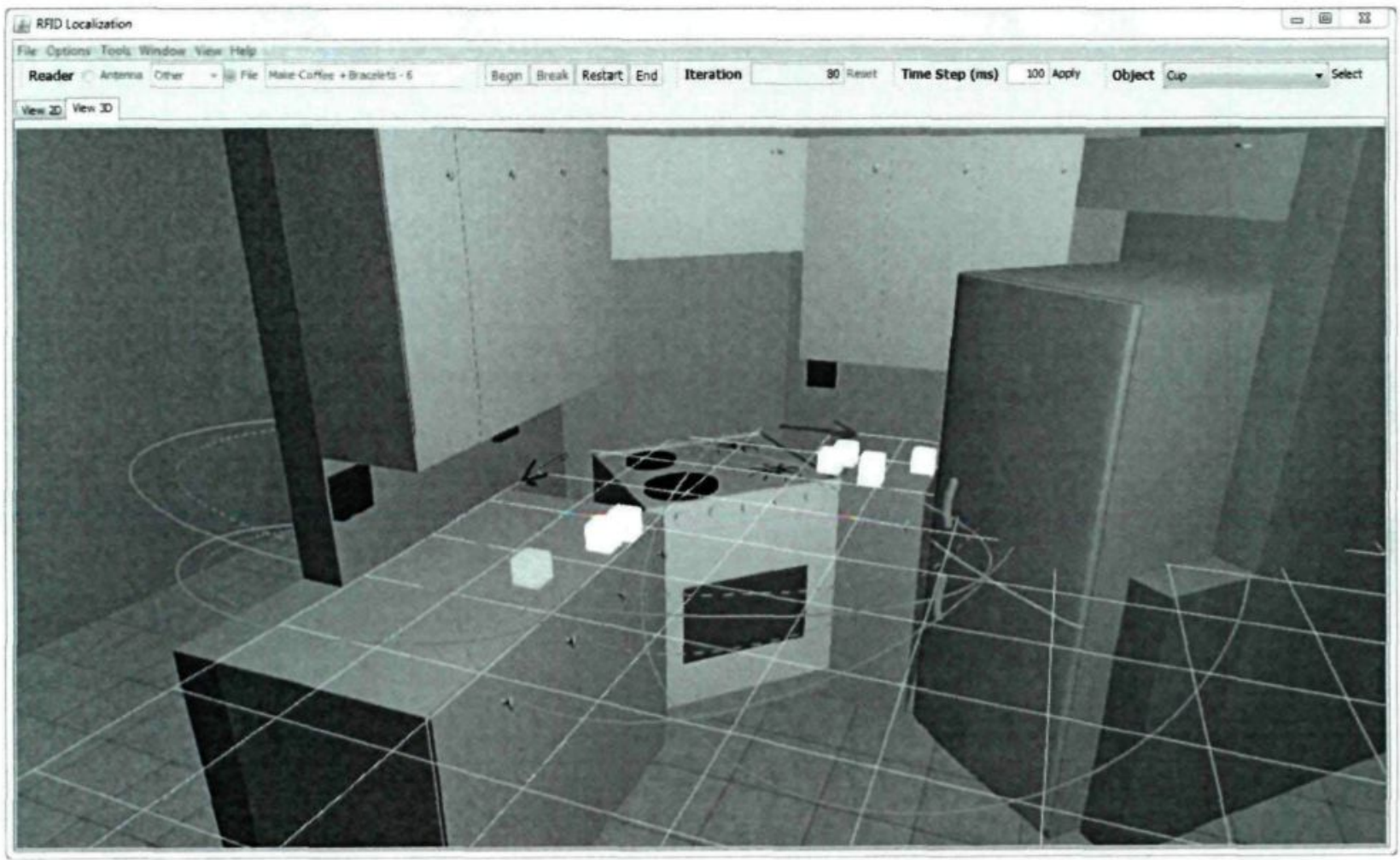

Figure 4.5 : Interface utilisateur avec la vue $3 D$

\subsubsection{Système de calibration}

Chaque partie de l'algorithme a besoin d'une configuration. Les paramètres des algorithmes et des filtres utilisés nécessitent un ajustement précis pour avoir une précision optimale. Bien qu'ils puissent être estimés, l'outil de calibration permet d'obtenir facilement les paramètres optimaux du système de positionnement dans des contextes variés et avec des configurations matérielles différentes. Pour ce faire, nous avons enregistré les données RSSI et les trajets de références (réels) des expérimentations effectuées (voir la section 4.3) dans notre environnement domestique intelligent. Ensuite, l'outil de calibration teste toutes les 
combinaisons de notre modèle de multilatération elliptique en augmentant progressivement la valeur des paramètres. Le logiciel calcul la précision moyenne pour chaque configuration ou combinaison de paramètres. Par la suite, il sélectionne la valeur des paramètres qui optimise la précision du positionnement. De plus, comme on peut le voir aux figures 4.6 et 4.7, l'outil de calibration permet ainsi de passer d'une précision moyenne de $14.40 \mathrm{~cm}$ à $11.64 \mathrm{~cm}$ lors des expérimentations sur un objet statique, et de $16.08 \mathrm{~cm}$ à $14.23 \mathrm{~cm}$ lors des expérimentations sur un objet en mouvement. Donc, les résultats d'expérimentation de la section subséquente sont ceux obtenus avec les paramètres optimaux sélectionnés avec l'outil de calibration.

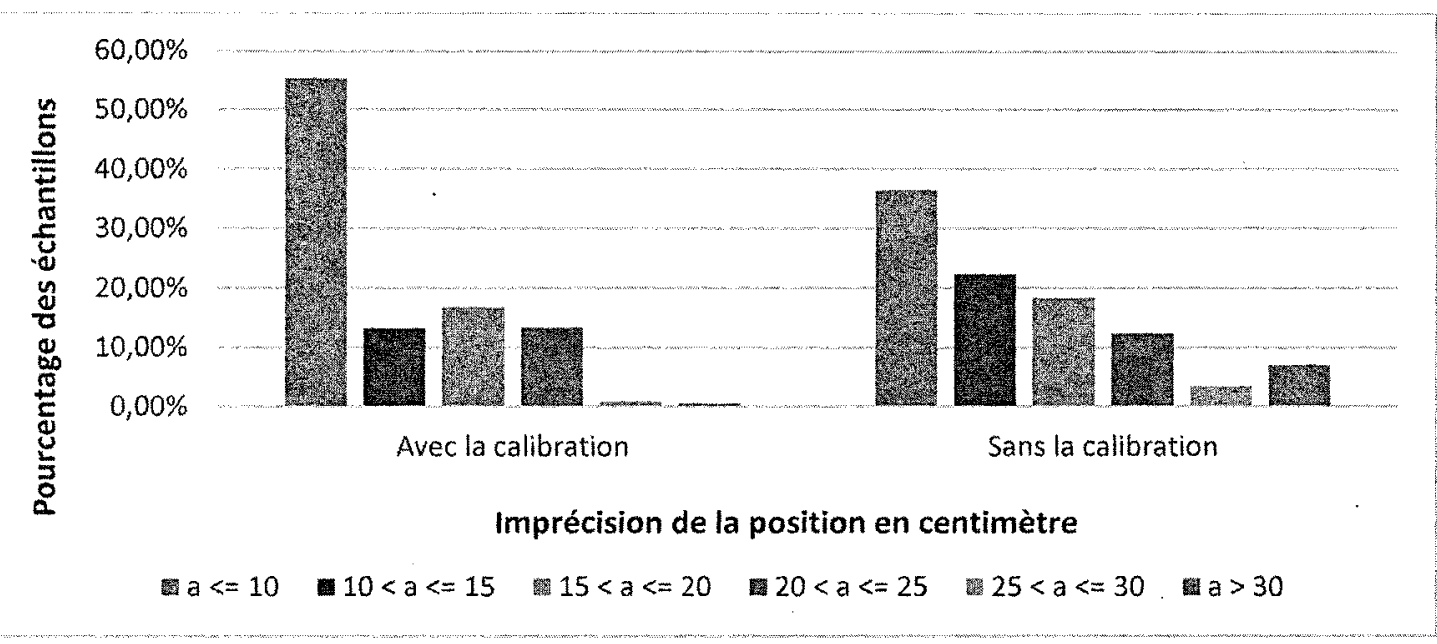

Figure 4.6: Amélioration de la précision d'un objet statique avec l'outil de calibration

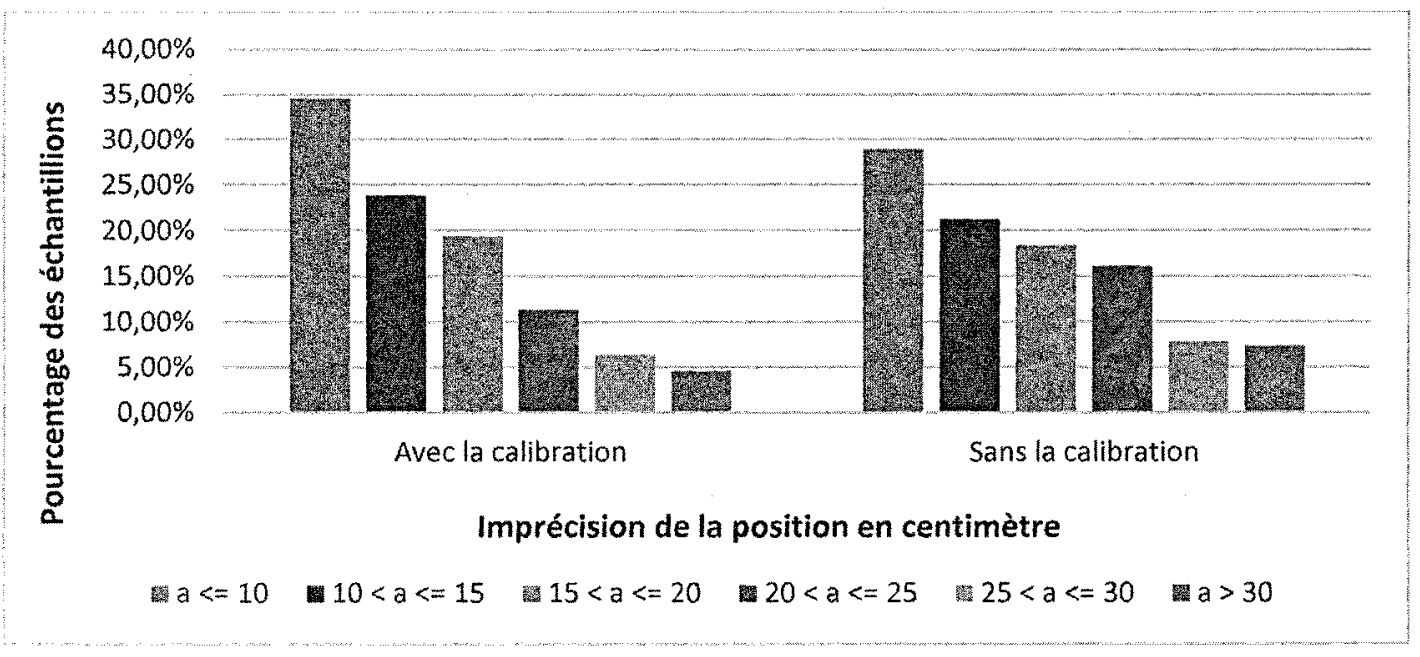

Figure 4.7: Amélioration de la précision d'un objet dynamique avec l'outil de calibration 


\subsection{Expérimentations}

Cette section décrit les scénarios réalistes que nous avons construits pour mettre à l'épreuve le nouveau modèle de localisation. De plus, le potentiel d'exploitation du système de positionnement a été vérifié par l'expérimentation de celui-ci à l'intérieur d'un modèle de reconnaissance d'activité dans le contexte d'un habitat intelligent. Par ailleurs, la flexibilité du système a été mise à l'épreuve lors d'une collaboration avec une entreprise ayant pour but de résoudre leurs problèmes et, donc, de produire un système représentant plus fidèlement les déplacements d'une personne dans un environnement extérieur (parc, festival, etc.). Cette partie « transfert technologique » a également permis de montrer l'applicabilité de notre modèle à d'autres contextes que celui de l'habitat intelligent.

\subsubsection{Protocole d'expérimentation au LIARA}

Nous avons effectué deux séries d'expérimentations. La première avait pour but de valider l'efficacité des différents filtres de correction d'erreur des forces de signaux (RSSI) avec un objet statique ne se déplaçant pas. La deuxième avait pour objectif de tester la méthode de correction des déplacements d'un objet en mouvement, ainsi que de mettre à l'épreuve le système dans sa globalité.

\subsubsection{Expérimentation de la localisation avec un objet statique}

Pour tester la précision de l'algorithme de localisation, nous avons utilisé une tasse de café avec quatre étiquettes pointant dans différentes directions afin d'être en mesure de bien capter les signaux provenant de toutes les directions. Cette tasse a été placée à divers endroits dans la cuisine pendant 300 itérations à la même hauteur que les antennes pour éliminer la troisième dimension du problème. Les positions réelles ont été mesurées avec précision à 
l'avance pour permettre le calcul de l'imprécision moyenne du système de positionnement dont les résultats sont discutés à la prochaine sous-section.

\subsubsection{Résultats de la localisation avec un objet statique}

La multilatération elliptique a été testée sans les filtres et a donné une précision moyenne de $15.79 \mathrm{~cm}$. Nous avons ensuite testé la multilatération avec le filtre de réduction des fausses lectures et une précision de $13.75 \mathrm{~cm}$ a été obtenue. En ajoutant le filtre de pondération par moyenne gaussienne, une précision de $12.6 \mathrm{~cm}$ a pu être obtenue. Enfin, l'ajout des règles du comportement de mouvement a permis de passer à précision de $11.64 \mathrm{~cm}$.

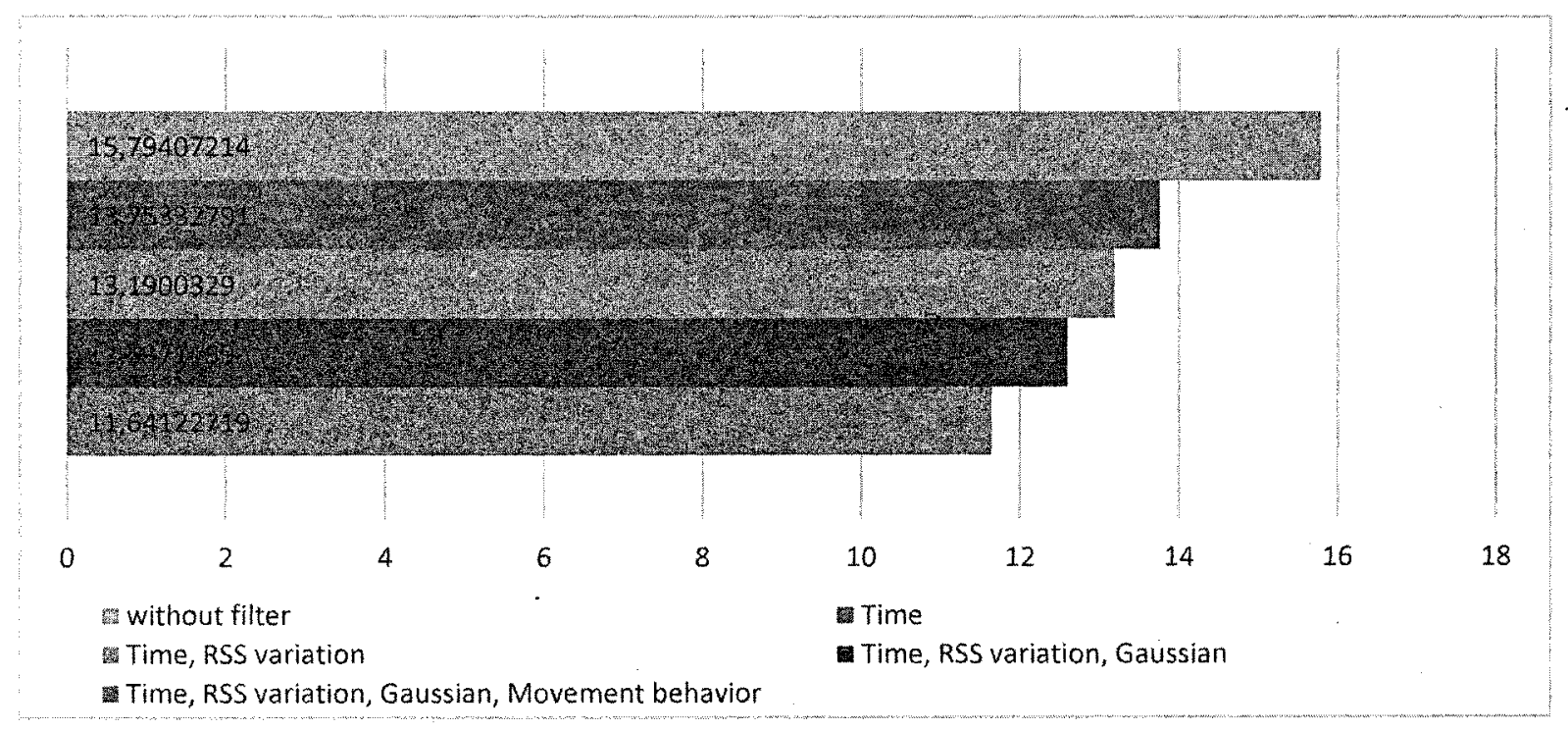

Figure 4.8: Précision sous différentes configurations

À la figure 4.9 ci-dessous, on peut conclure de la pertinence des filtres proposés en voyant la prévision de la localisation augmenter au fur et à mesure qu'on ajoute un filtre. De plus, avec l'activation de tous les filtres, les erreurs de positionnement au-dessus de $25 \mathrm{~cm}$ sont presque totalement éliminées et la majorité des positions calculées sont dans un rayon inférieur à $10 \mathrm{~cm}$ de la position réelle. Ces résultats sont très prometteurs et se comparent avantageusement à ce qui se fait dans la littérature. En ce sens, notre approche est très compétitive. 


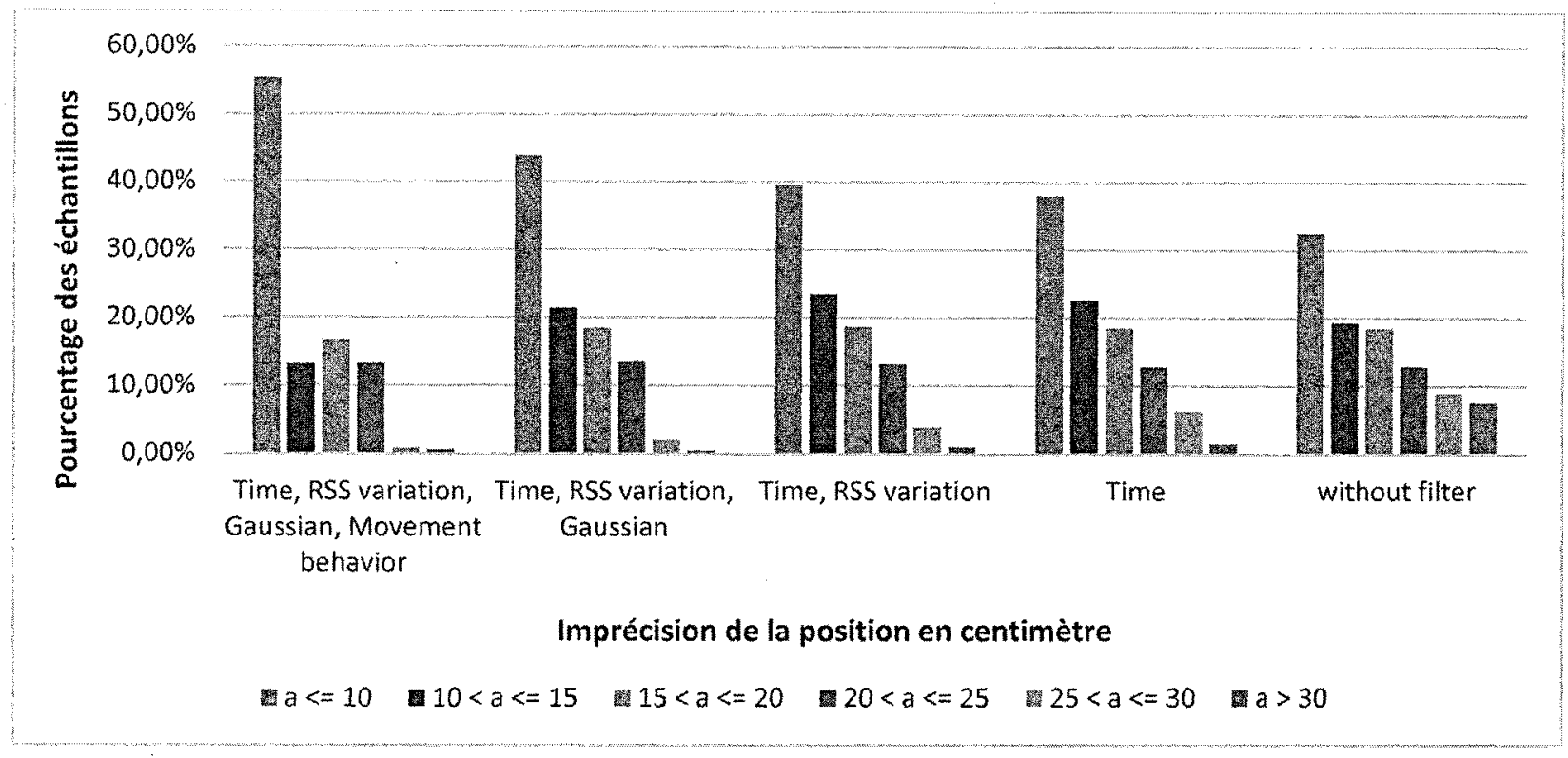

Figure 4.9: Répartition de l'imprécision sous différentes configurations

A la figure 4.10 , on voit bien le résultat représenté à la figure 4.9 où les positions calculées dont l'erreur se situe entre 10 et $15 \mathrm{~cm}$ sont corrigées de telles sortes que leurs imprécisions deviennent inférieures à $10 \mathrm{~cm}$. Donc, en pratique, les faux déplacements sont grandement diminués, ce qui peut permettre de détecter plus facilement si l'objet est actif ou inactif (s'il se déplace ou non).
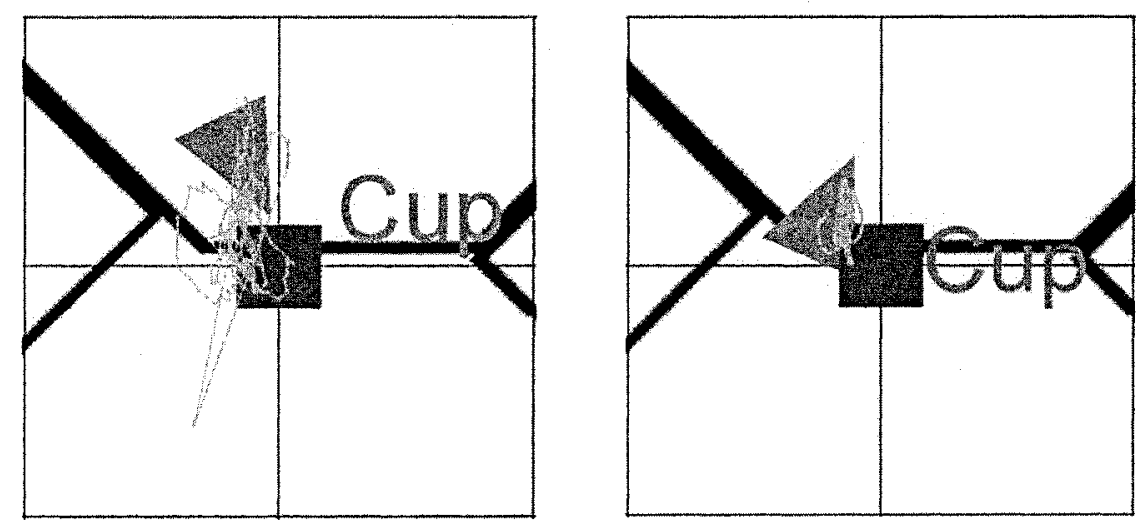

Figure 4.10: L'image à gauche est obtenue sans le comportement de mouvement et l'image à droite est obtenue en utilisant le comportement de mouvement. 
La première chose que nous pouvons voir, c'est que la précision s'améliore avec l'ajout de chaque filtre. En fait, nous étions très satisfaits de constater que même le comportement de mouvement a amélioré la précision d'un objet stationnaire alors qu'il n'était pas prévu pour cela. Après une analyse complète de ces résultats, nous pouvons conclure que chacun des différents filtres joue bien leur rôle efficacement et leur combinaison offre une bonne stabilité ainsi qu'une bonne précision.

\subsubsection{Expérimentation de la localisation avec un objet en mouvement}

Pour tester la précision de l'algorithme de localisation avec un objet en mouvement, nous avons utilisé une tasse de café avec quatre étiquettes. Cette tasse se déplace en suivant des trajets prédéfinis dans la cuisine pendant environ 600 itérations. L'objet se déplace à vitesse constante et les positions réelles de départ, d'arrivée, ainsi que les positions intermédiaires ont été mesurées à l'avance.

\subsubsection{Résultats de la localisation avec un objet en mouvement}

À la figure 4.11 ci-dessous, on peut s'apercevoir que l'utilité des différents filtres appliqués sur les forces de signaux bruts est restreinte dans le cas où l'objet étiqueté est en mouvement. D'ailleurs, c'est ce qui a motivé l'ajout d'un filtrage des positions calculé à partir de ces forces de signaux bruitées. De plus, à l'égard des résultats obtenus l'ajout de règles régissant le comportement de mouvement des objets s'est avéré plus que nécessaire. 


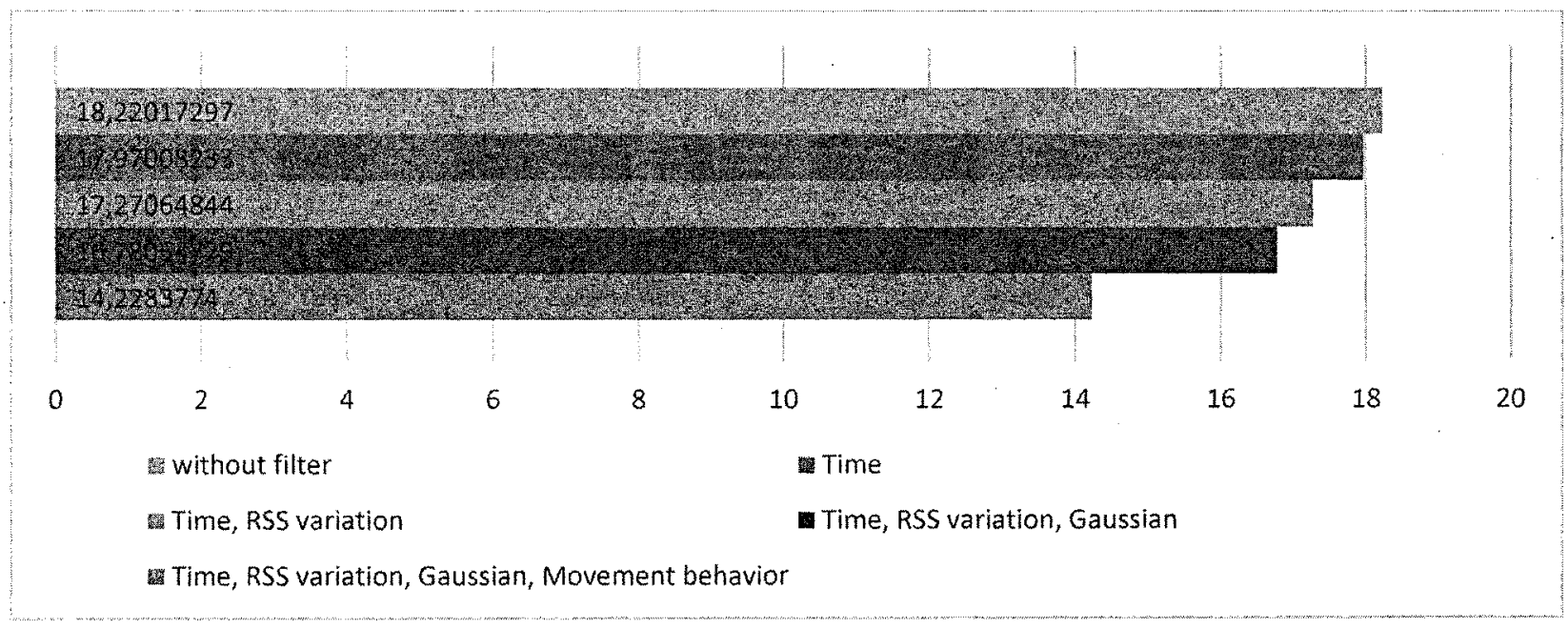

Figure 4.11: Précision sous différentes configurations avec un objet en mouvement

Par ailleurs, les résultats illustrés à la figure 4.12 confirment l'analyse plus générale. Donc, l'inefficacité des différents filtres appliqués sur les RSSI est confirmée et les erreurs de positionnement et la répartition de ces erreurs restent tout aussi aléatoires que sans l'utilisation de ces filtres. À l'opposé, les résultats détaillés sur le comportement de mouvement permettent de confirmer son efficacité. Il enlève un aspect aléatoire dans la répartition des résultats et met en évidence la diminution du nombre de données les plus imprécises (supérieur à $20 \mathrm{~cm}$ ).

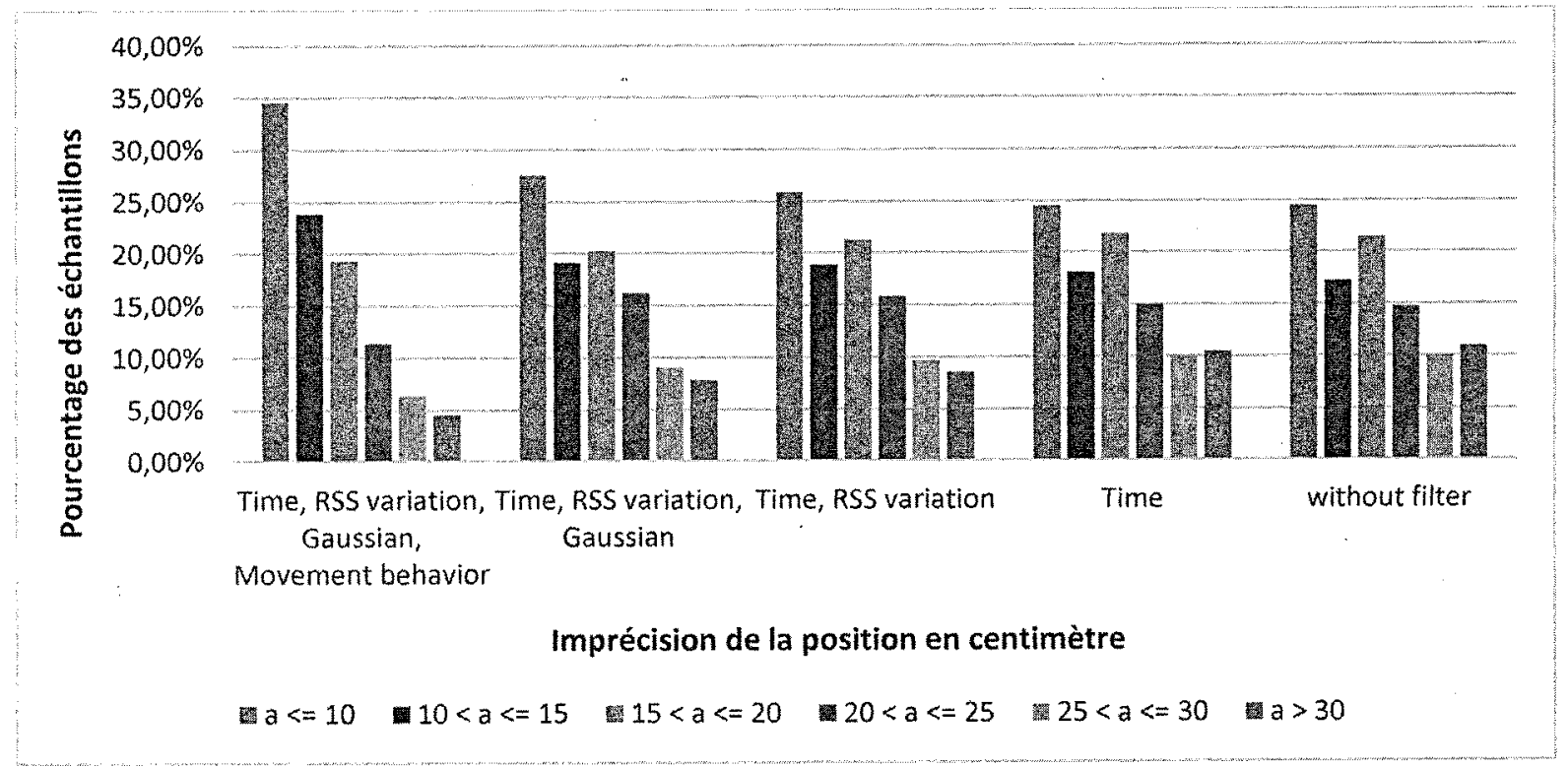

Figure 4.12: Répartition de l'imprécision sous différentes configurations avec un objet en mouvement 
Dans le but de confirmer encore plus l'utilité du filtre appliqué sur les positions, une analyse plus poussée a été effectuée. La figure 4.13 représente le trajet parcouru par un objet lors d'une des expérimentations. À partir de cette figure comparative, on peut voir qu'en pratique, la trajectoire est grandement améliorée. Par ailleurs, cette amélioration peut permettre de détecter plus facilement les gestes effectués par l'usager. À ce titre, on note qu'une des avenues d'utilisation envisagée pour le système de localisation en temps réel concerne la reconnaissance de gestes simples (brasser le contenu d'une casserole, déposer un objet, prendre, etc.). Conséquemment, notre système permet de faire un pas en avant vers cet objectif en augmentant la précision et la stabilité du processus de positionnement RFID.
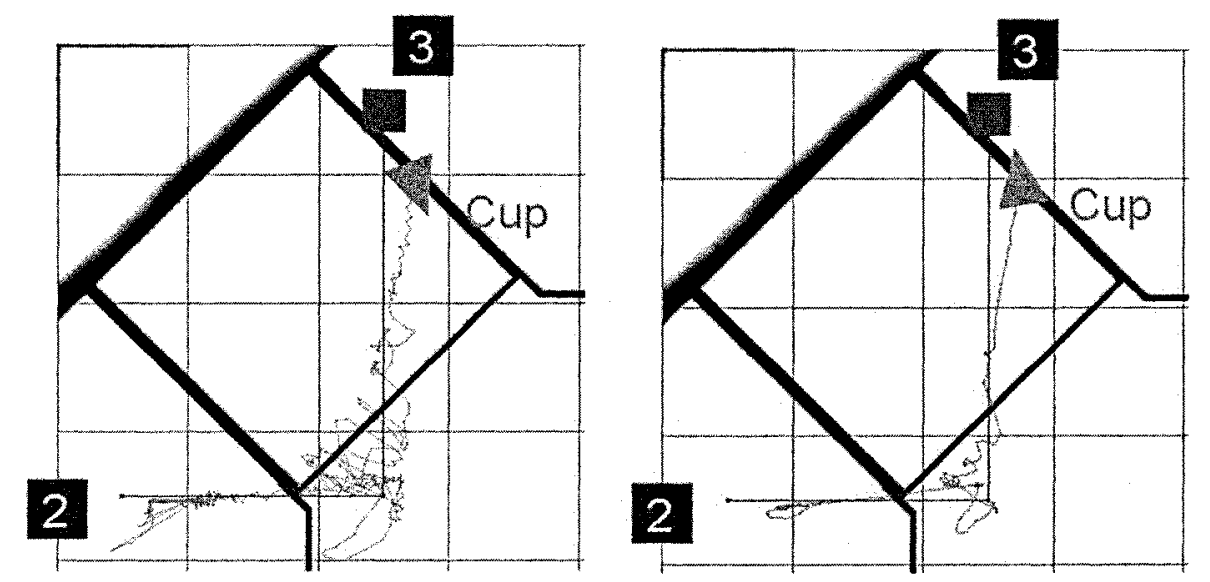

Figure 4.13 : L'image à gauche est obtenue sans le comportement de mouvement et l'image à droite est obtenue en utilisant le comportement de mouvement.

Pour conclure, on peut noter que les règles de déplacement augmentent de manière significative la précision du positionnement des objets. Ce filtre a un impact considérable.

\subsubsection{Expérimentation avec le système de reconnaissance de comportements erronés}

La figure 4.14 montre en action un module de reconnaissance d'activités simples que nous avons mis en œuvre avec le système de positionnement. L'objectif de cette expérimentation 
était de montrer l'applicabilité du nouveau système de positionnement dans le contexte visé de reconnaissance d'activités, d'identification de l'étape courante, et dans la détection d'erreurs (inversion d'étapes, saut d'une étape, délai trop long entre deux étapes, etc.). Pour ce faire, nous avons sélectionné deux activités de la vie quotidienne (MakeCoffee et MakeSpaghetti) pour lesquelles nous avons défini la séquence correcte des interactions spatiales entre les objets. Il s'agit d'activités standards couramment utilisées dans les tests en laboratoire. Ces AVQ ont été simulés par un sujet humain dans l'habitat intelligent à dix reprises chacune, sans y incorporer d'erreurs. Sur la séquence des 20 essais, toutes les étapes ont été reconnues dans le bon ordre. Par la suite, nous avons simulé à nouveau ces deux AVQ, mais cette fois en y ajoutant des erreurs provenant des quatre types mentionnés. Un total de huit réalisations erronées a été réalisé et l'algorithme les a toutes correctement identifiées.

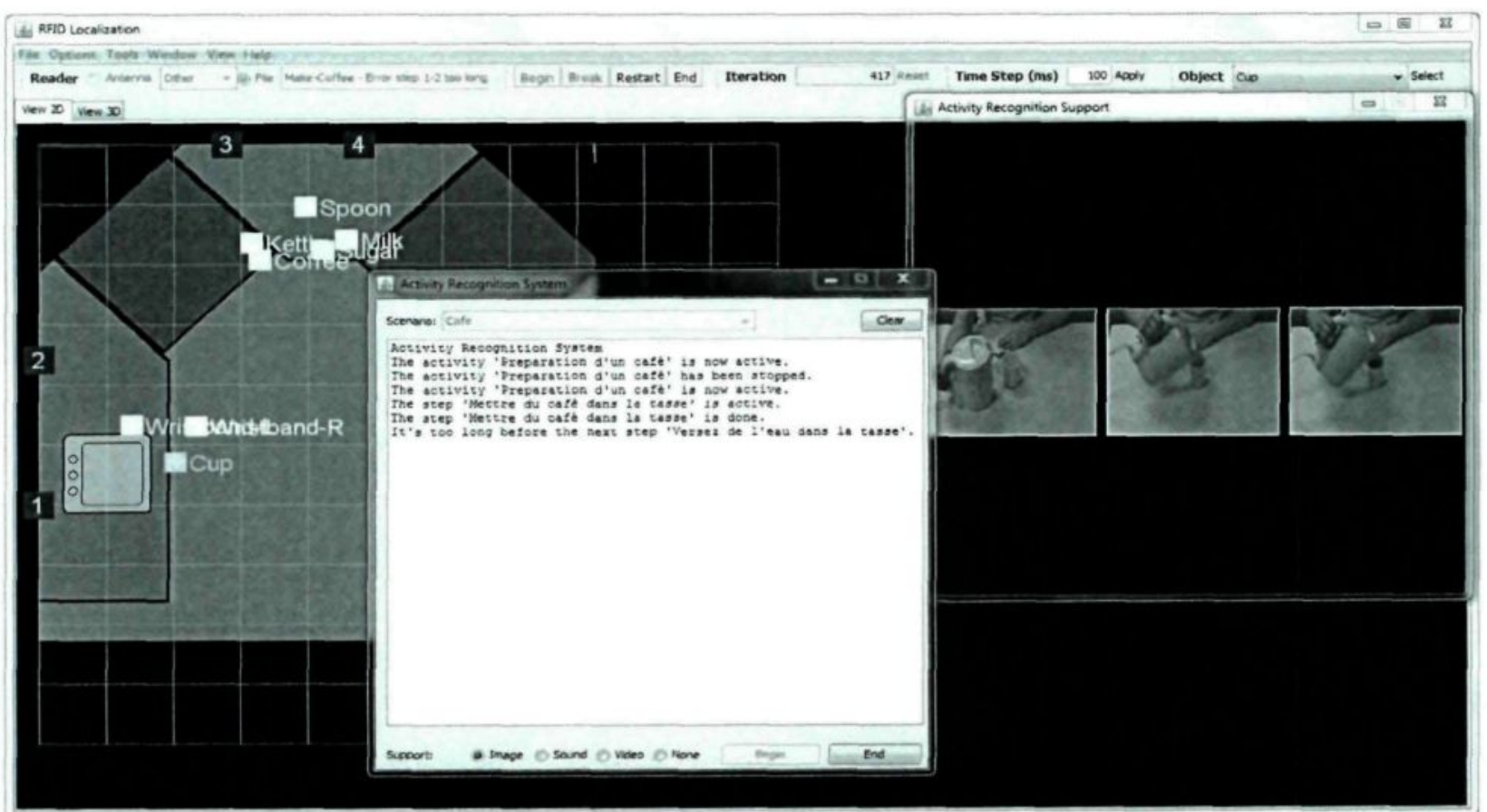

Figure 4.14: Fonctionnement du module de reconnaissance de comportements erronés 
Bien que ces tests soient préliminaires et n'étaient pas, pour ainsi dire, une composante directe de ce projet de recherche, ils démontrent tout de même le potentiel d'applicabilité de l'approche algorithmique proposée dans un contexte de reconnaissance d'activités. La technologie RFID est souvent présentée, en raison de sa robustesse et de son faible coût, comme l'un des principaux outils qui permettront éventuellement la mise en œuvre de système de suivi d'activités et d'assistance à domicile. En ce sens, ces tests préliminaires montrent que le système de positionnement peut être utilisé efficacement pour la reconnaissance d'étapes d'une activité et des erreurs. C'est donc un pas dans la bonne direction. Il montre que notre système peut contribuer à terme à mise en place d'une telle technologie.

\subsubsection{Contribution (transfert technologique) à l'industrie (TLM)}

Solutions TLM, entreprise établie à Chicoutimi, est spécialisée dans les services d'infrastructure technologiques. Parmi ses produits, l'entreprise offre une solution RFID (via des balises actives) pour les festivals: bracelets RFID pour les festivaliers, réseau d'antennes, contrôle d'accès et analyse de données. Cette solution est notamment exploitée dans le cadre des Festivals de Québec et de Chicago. Récemment, l'entreprise a développé un système novateur de traçabilité des festivaliers. Ce système positionne, avec comme seule information la force du signal reçu via un réseau d'antennes implantées sur le site, le participant à partir d'un signal radio émis par une étiquette RFID incorporée à son bracelet d'admission. Ceci est fait pour l'ensemble des festivaliers. Par la suite, les données amassées sont utilisées pour établir les modifications à apporter au site pour assurer la fluidité des déplacements des participants et ensuite pour étudier les profils de consommation (achat d'articles promotionnels, publicité, etc.) des participants en tenant compte de l'aménagement du site. L'entreprise, dans un souci de proposer des systèmes toujours plus performants à ses clients, a fait appel à notre équipe. Cette collaboration s'est traduite par un projet de six mois de R\&D visant le transfert/l'adaptation de 
notre expertise dans le ce domaine ciblé afin d'améliorer le système de l'entreprise. Il a été financé par une subvention d'engagement partenarial (SEP) du CRSNG.

L'objectif du projet visait d'améliorer la précision et l'efficacité du système de traçabilité par radio-identification de l'entreprise afin de disposer de données plus significatives dans le contexte de la gestion du personnel sur le site, ainsi que des festivaliers. Dans ce contexte, notre approche de localisation a été adaptée et déployée avec des balises actives dans un environnement extérieure, ce qui a permis la mise à l'épreuve de l'approche dans un contexte totalement différent (balises actives, faible taux d'émission, environnement extérieur, etc.).

\subsubsection{Expérimentation}

Tout d'abord, des expérimentations ont été menées en extérieur sur le terrain de football de l'UQAC pour recueillir l'expérience ainsi que les données nécessaires avant les expérimentations à plus grande échelle effectuées dans un cadre réaliste. Nous avons commencé par effectuer le test de propagation des ondes selon la distance. Ensuite, nous avons procédé à l'enregistrement de plusieurs scénarios (trajets) avec un sujet humain se déplaçant à vitesse constante avec des balises RFID actives se rafraichissant toutes les 5 secondes. La nature du terrain et le déplacement à vitesse constant ont facilité la prise de données sur le trajet réel comme, par exemple, le moment où la balise RFID atteint certaines positions du trajet. La figure 4.15 montre la configuration matérielle de cette expérimentation préliminaire qui a été réalisée avec 8 antennes placées sur un terrain de football d'une superficie d'environ $6000 \mathrm{~m}^{2}(60 \mathrm{~m} \mathrm{x}$ $100 \mathrm{~m})$. 


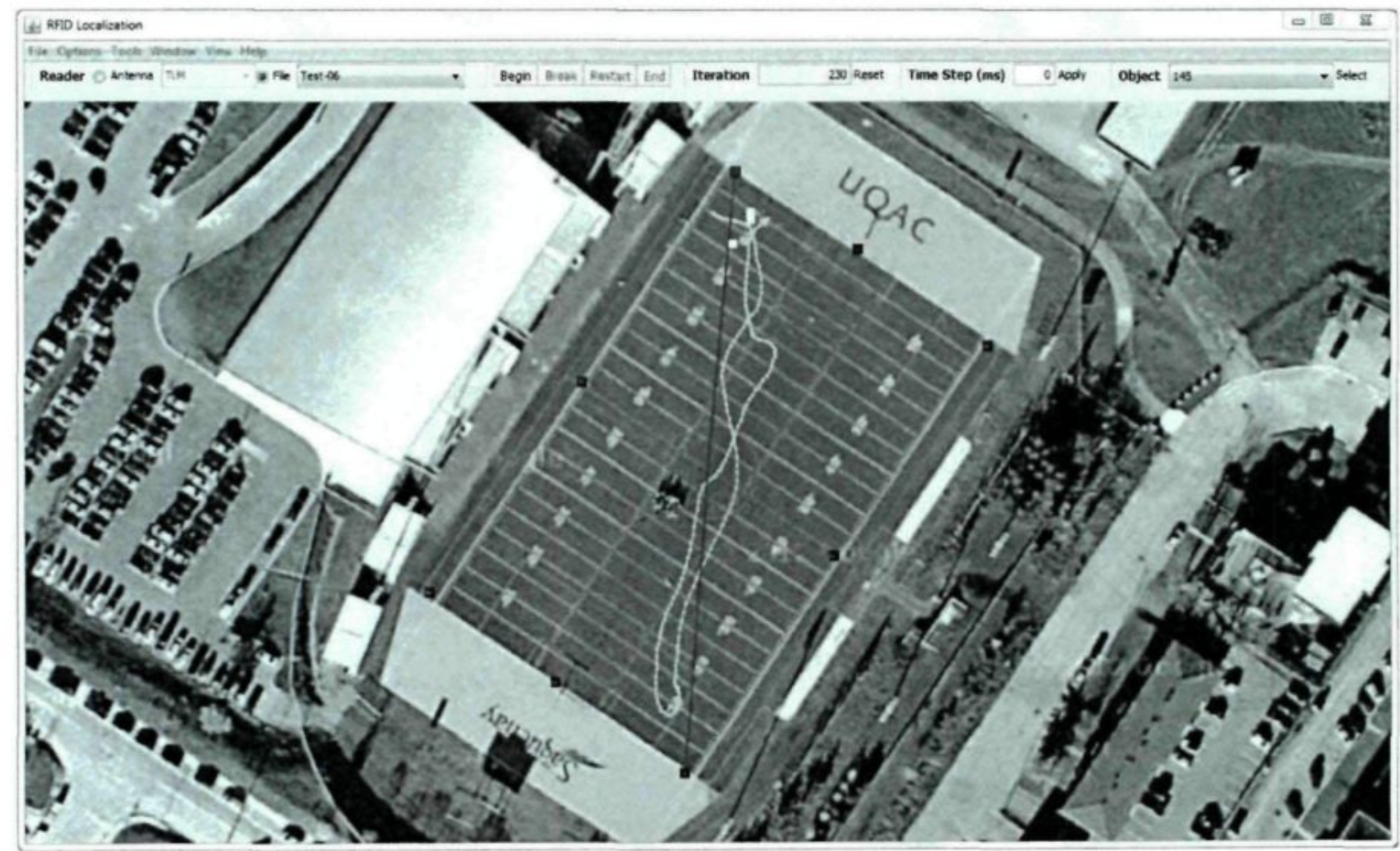

Figure 4.15: Fonctionnement du système de localisation avec du matériel RFID actif en extérieur. Le trajet de référence est en rouge et le trajet calculé avec l'algorithme de positionnement est en jaune. Les carrés en bleu représentent des antennes RFID.

Ensuite, dans le but de mettre à l'épreuve le système dans des conditions plus réalistes nous avons procédé à des expérimentations sur une zone portuaire d'une superficie d'environ $55200 \mathrm{~m}^{2}(460 \mathrm{~m} \times 120 \mathrm{~m})$ sur lequel on a réparti 16 antennes à des emplacements stratégiques tels qu'illustrés à la figure 4.16. Ces expérimentations consistent à l'enregistrement de scénarios où un sujet humain se déplace avec des balises RFID actives se rafraichissant toutes les 10 secondes contrairement à ceux des expérimentations précédentes avec un taux d'émission plus rapide et plus adapté à la problématique (aux 5 secondes). Contrairement à l'expérimentation préliminaire, l'utilisation de la technologie GPS a été nécessaire pour obtenir les trajets de référence. Finalement, ces trajets de référence ont servi à évaluer la précision du système de positionnement et l'efficacité relative du matériel à faible coût utilisé comme l'illustre la figure 4.17. 


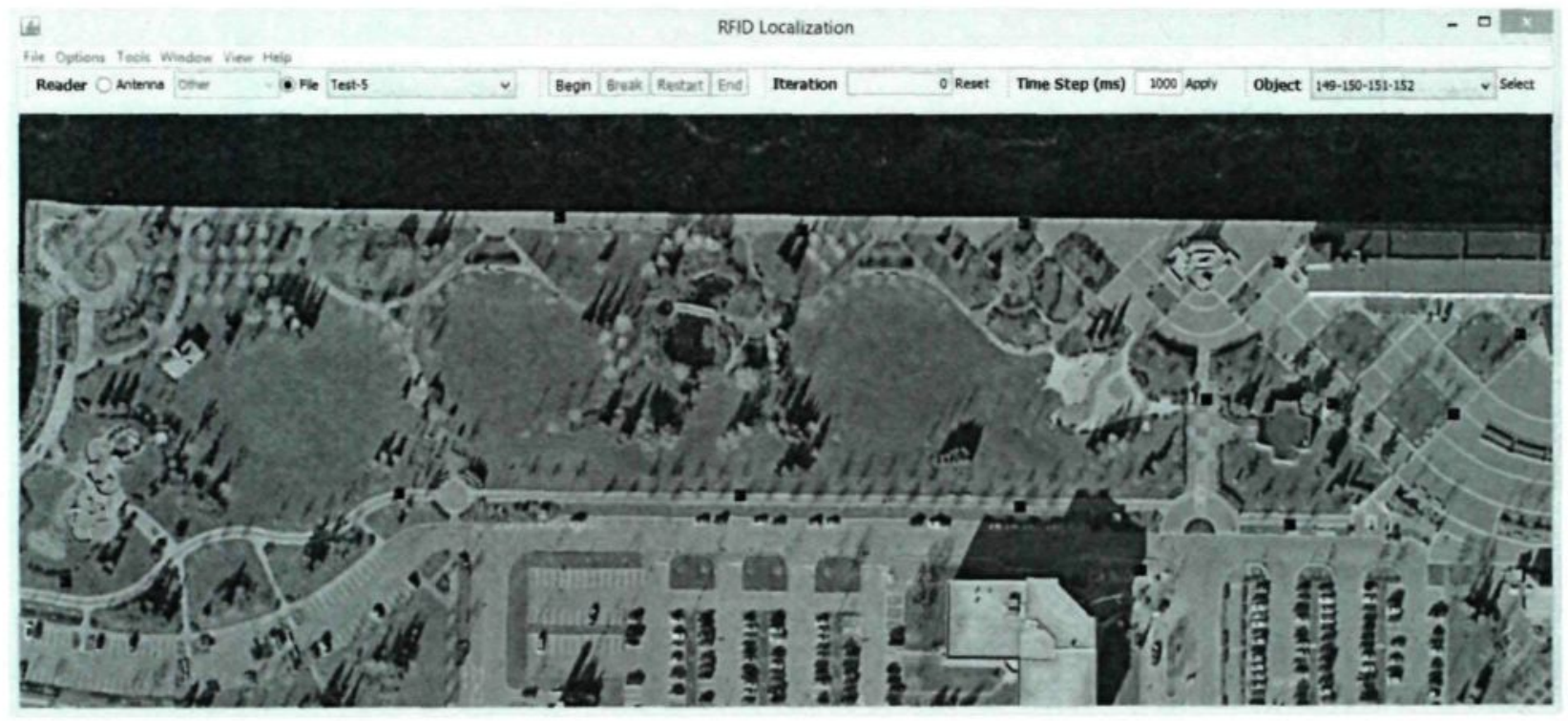

Figure 4.16 : Emplacement des 16 antennes RFID (les carrés en bleu) lors des expérimentations à la zone portuaire.
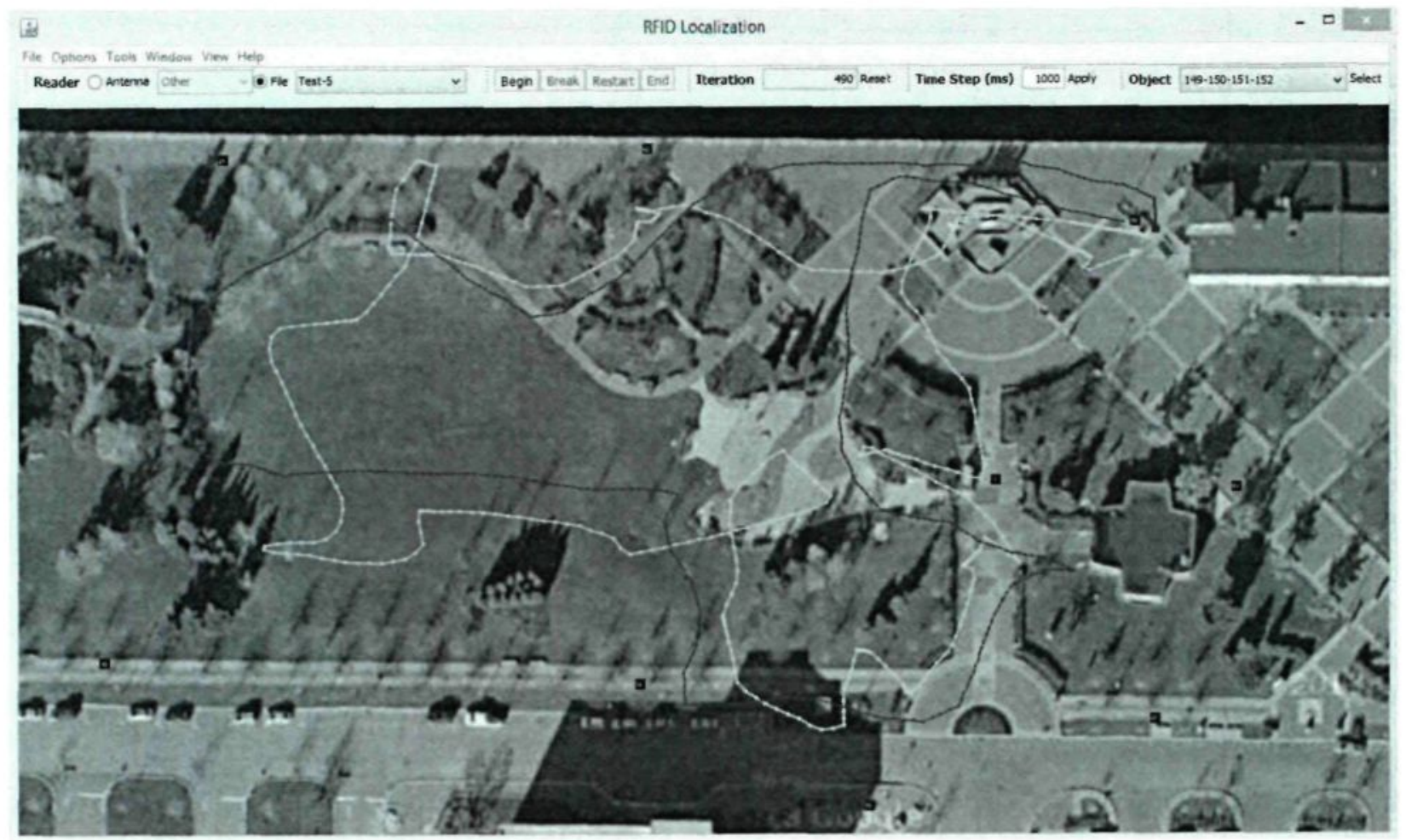

Figure 4.17 : Fonctionnement du système de localisation avec du matériel RFID actif en extérieur. Le trajet GPS est en rouge et le trajet calculé avec l'algorithme de positionnement est en jaune. Les carrés en bleu représentent des antennes RFID. 


\subsubsection{Résultats}

Premièrement, les résultats obtenus lors des expérimentations sur le terrain de football sont plus que satisfaisants. Avec une erreur moyenne de seulement 10.09 mètres, nous ne pourrions pas espérer mieux. Sans oublier qu'à $93.52 \%$ du temps, nous respectons le standard qui est d'être à moins de 20 mètres de la position réel. Cependant, ces résultats ont été obtenus dans des conditions idéales sur un terrain sans obstacle avec une densité d'une antenne aux $750 \mathrm{~m}^{2}$. Pour finir, on peut une fois de plus valider l'utilité de chacun des filtres en analysant la précision sous différentes configurations tel qu'illustrée à la figure 4.18 .

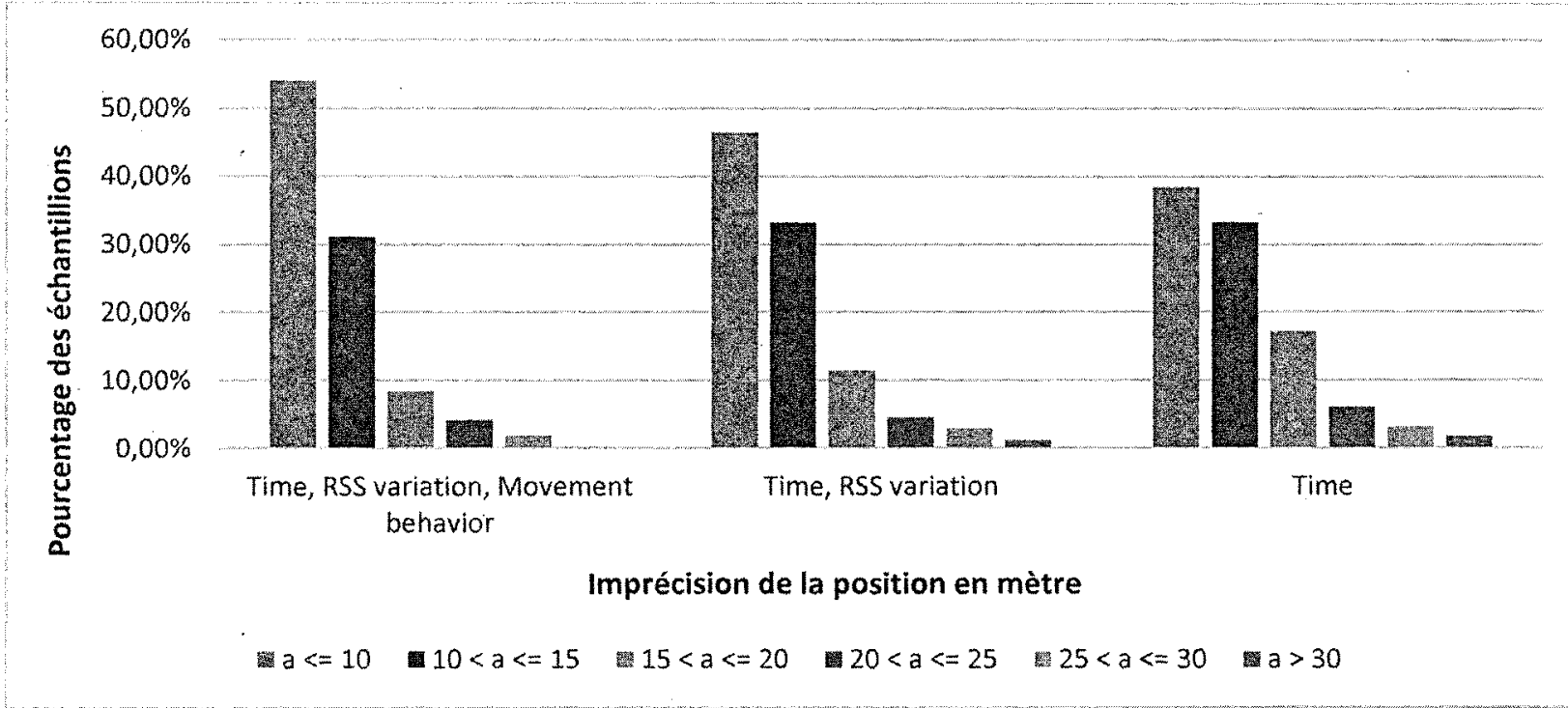

Figure 4.18: Répartition de l'imprécision sous différentes configurations lors des expérimentations sur le terrain de football.

Deuxièmement, les résultats obtenus lors des tests à la zone portuaire sont presque tout autant satisfaisants malgré tous les problèmes matériels survenus lors de ces expérimentations. Tout d'abord, les balises utilisées ont une rapidité d'émission deux fois plus faible. Ensuite, certaines antennes ne répondaient que très rarement alors qu'en majorité le taux de réponse était faible (une balise RFID pouvait fréquemment ne plus être captée pendant 40 secondes par une ou plusieurs antennes). Enfin, la densité des antennes était plutôt faible avec en moyenne une 
antenne aux $3450 \mathrm{~m}^{2}$ environ. Pour conclure, en considérant tous ces facteurs, nous ne pourrions pas espérer beaucoup mieux qu'une erreur moyenne de 30.89 mètres avec une probabilité de $32.89 \%$ que la position calculée soit à moins de 20 mètres de la position GPS puisque la densité des antennes sur ce terrain est de 4.6 fois plus faible.

En conclusion, notre système s'est avéré flexible en plus d'être facile et rapide à configurer dans ce contexte totalement différent (balises actives, faible taux d'émission, environnement extérieur, etc.). Par ailleurs, la mise à l'épreuve de l'approche dans ce contexte différent à conduit à l'ajout du filtre de réduction de crêtes (la moyenne gaussienne est totalement inutilisable avec un taux d'émission si faible) en plus de prouver une fois de plus la nécessité d'un filtre appliqué sur les positions qui a pour effet de grandement améliorer le trajet parcouru dans ce contexte. Enfin, on peut conclure que l'objectif du projet qui visait l'amélioration de la précision a été réalisé avec succès.

\subsection{Analyse et comparaison avec les résultats présentés dans les ouvrages connexes}

La comparaison entre nos résultats expérimentaux et ceux présentés dans les travaux connexes n'est pas si simple si l'on considère qu'ils ont des contextes d'expérimentation bien différents. Cependant, nous pouvons tout de même comparer les différents systèmes sur certains de leurs aspects (le matériel utilisé, la vitesse approximative, la superficie couverte et la précision). Le tableau 4.1 ci-dessous présente un résumé de la comparaison entre les principaux systèmes de positionnement. 


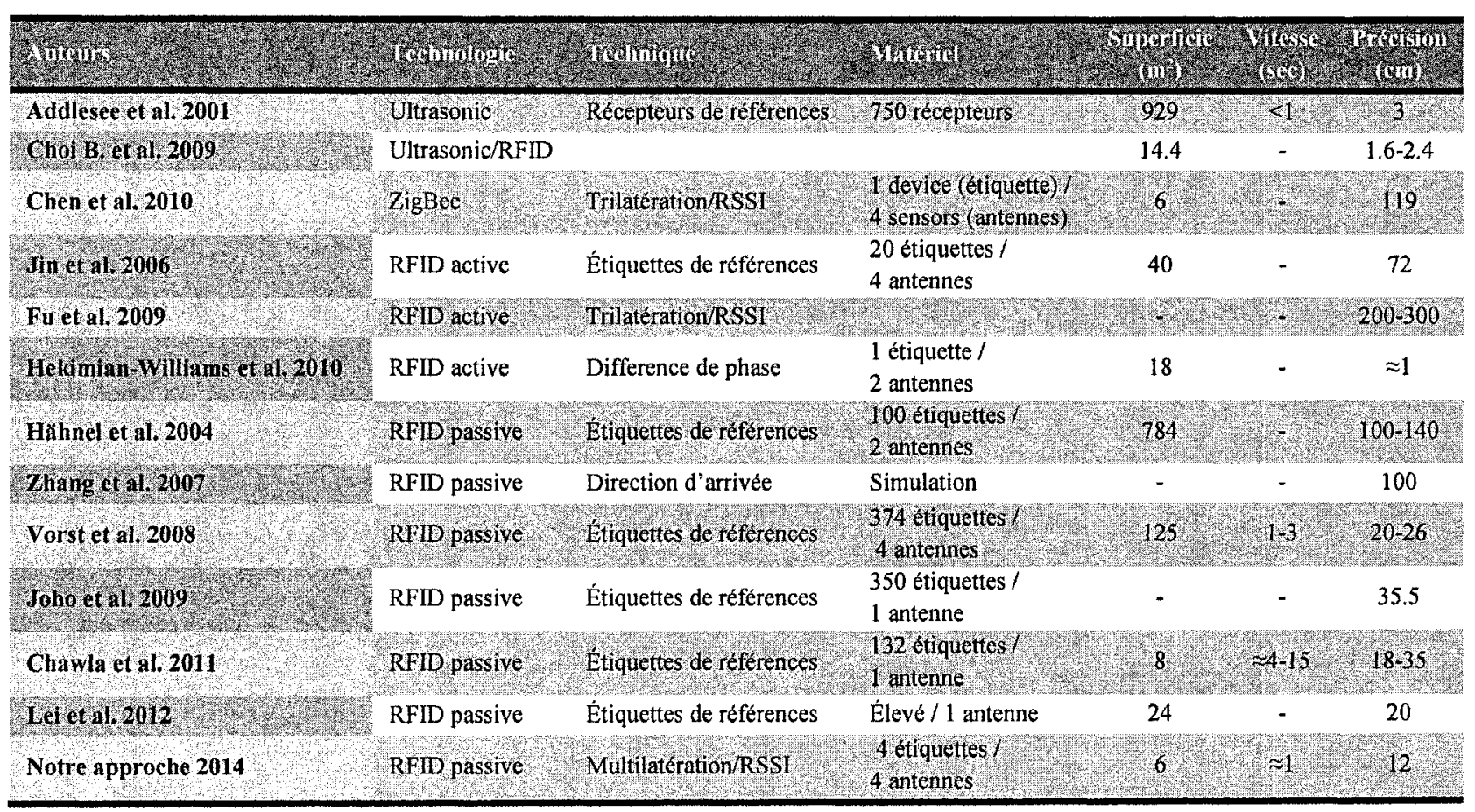

Table 4.1 - Tableau récapitulatif des différents systèmes de localisation.

\subsubsection{Matériel}

Tout d'abord, notre modèle nécessite moins de matériel à déployer pour être fonctionnel par rapport à d'autres approches puisque nous n'avons pas utilisé des balises de référence. Sans la nécessité de ces balises de référence, notre système est beaucoup plus facile à déployer dans un nouvel environnement en évitant leur installation et leur étalonnage ultérieur. Pour conclure, notre système donne des résultats prometteurs et précis tout en évitant l'utilisation de cette technique d'amélioration de la précision.

\subsubsection{Vitesse}

Ensuite, la vitesse est aussi un autre critère très important pour évaluer la performance d'un système de localisation. Nous pouvons calculer la position de plusieurs objets sans difficulté et en simultané en quelques millisecondes. En pratique, le calcul de la position des objets est répartir entre les processeurs disponibles et la vitesse du système est en définitive limitée par le matériel RFID qui est synchronisé de manière à lire toutes les étiquettes à toutes les 
100 millisecondes. Par contre, le filtre de pondération par moyenne gaussienne provoque un certain décalage qui a en quelque sorte pour effet de produire un ralentissement de la vitesse de mise à jour à environ une seconde. Pour finir, à partir des données disponibles sur la vitesse de mise à jour de la position, le système se classe parmi les plus rapides.

\subsubsection{Superficie et précision}

Finalement, avec une précision de $11.64 \mathrm{~cm}$ sur une superficie de $6 \mathrm{~m}^{2}$ on peut conclure que notre système de localisation est plus précis que les systèmes comparables (ceux qui utilisent la trilatération). De plus, notre système parvient à être plus précis que la majorité des autres systèmes qui sont moins flexibles et qui présentent des inconvénients (besoin de piles, le coût, les dimensions, etc.) qui les rendent difficiles à utiliser dans le contexte d'un habitat intelligent.

\subsection{Conclusion}

Dans ce chapitre, nous avons décrit nos plus récents progrès vers le développement d'un système de localisation en temps réel basé sur la technologie RFID passive. Nous avons montré comment chaque filtre contribue à améliorer la précision avec des expériences effectuées dans le contexte réaliste de l'habitat intelligent. Ensuite, nous avons démontré que le comportement de mouvement traite d'un problème crucial dans le processus de localisation. De plus, nous avons expliqué comment réaliser la modélisation des AVQ avec simplement des relations topologiques qui existent entre les objets physiques. Par ailleurs, nous avons démontré par la contribution à l'industrie que notre système de localisation est grandement flexible et applicable dans des contextes variés. 


\section{CHAPITRE 5}

\section{CONCLUSION GÉNÉRALE}

Le projet de recherche de ce mémoire qui est présenté au travers des quatre chapitres précédents apporte de nouvelles pistes de solution au problème décrit dans l'introduction. L'objectif principal de ce mémoire est de proposer et de formaliser un nouveau modèle multicouche et un nouvel algorithme de localisation précise des étiquettes RFID passives capable d'être appliqué dans un contexte de reconnaissance d'activités et de détection des erreurs pouvant être commises per un résident au sein d'un habitat intelligent. À travers le chapitre 2, nous sommes capables d'évaluer l'importance de réduire considérablement l'imprécision des forces de signaux par l'application d'un ensemble de filtres avant d'effectuer le calcul de la position par multilatération elliptique. Nous pouvons aussi voir au chapitre 2 que plusieurs modèles fournissent de bons indices sur la manière de s'y prendre afin d'effectuer un bon système de localisation en général. Cependant, ces modèles n'ont pas été construits avec l'intention de répondre au problème spécifique de la localisation d'objets mobiles à l'intérieur d'un habitat intelligent. Par conséquent, chacun de ces modèles présente des limitations importantes à l'égard de notre contexte applicatif, principalement au niveau de la précision et de la robustesse.

Ensuite, au chapitre 3, le nouveau modèle de positionnement d'étiquette RFID passive est présenté d'un point de vue théorique. Basé sur les techniques de localisation par trilatération, ce modèle de multilatération elliptique corrige le problème majeur résultant du fait que le modèle (pattern) de propagation des ondes est très rarement circulaire. De plus, il propose une solution au problème de l'imprécision des ellipses pouvant produire de nombreuses intersections entre les 
différentes courbes ou cercles en calculant l'intersection entre les surfaces elliptique. De plus, différentes techniques de filtrage sont proposées pour diminuer les erreurs au niveau des forces de signaux ou au niveau de la position et des déplacements des objets.

Pour finir, le quatrième chapitre examine la contribution du mémoire d'un point de vue pratique et expérimental.

\subsection{Objectifs réalisés}

Le premier objectif du projet de recherche consistait à acquérir des connaissances sur les différentes techniques de localisation utilisant la technologie RFID afin d'entrevoir les contraintes spécifiques et de dégager des pistes de solution pour l'exploitation du positionnement d'objets étiqueté par des puces RFID passives dans le cadre d'un habitat intelligent. En effet, une revue de la littérature a été effectuée concernant le problème de la localisation par puces RFID en général [16, 30], et plus particulièrement ceux utilisant des étiquettes passives [31, 32, 53].

Le second objectif consistait à formaliser un nouveau modèle de localisation par puces RFID passives pour résoudre les problèmes et répondre aux contraintes imposées dans le contexte d'un habitat intelligent. À cet effet, la méthode proposée et détaillée au chapitre 3 consiste en une multilatération par intersection de disques elliptiques et se veut une extension du positionnement par trilatération $[15,29,34,35]$ afin de le rendre plus précis et fiable tout en restant flexible et facile à déployer.

Le troisième objectif du projet consistait en une implémentation logicielle de ce nouveau modèle de localisation de puces RFID passive dans le contexte d'un habitat intelligent (le laboratoire LIARA) dans le but de valider ses performances et d'établir une base de comparaison avec les autres approches de positionnement et, en particulier, celles qui répondent aux mêmes 
contraintes liées à notre contexte applicatif. Cet objectif a été réalisé en développant un nouveau logiciel avec un langage de programmation orienté objet (Java) qui communique directement avec l'infrastructure à la fine pointe de la technologie de l'habitat intelligent du LIARA à l'intérieur de l'Université du Québec à Chicoutimi (UQAC).

Finalement, le dernier objectif du projet consistait en la validation du nouveau modèle créé et mis en œuvre. Le résultat est sans équivoque et démontre bien la nécessité de chaque partie ou filtre de ce nouveau modèle de positionnement. De plus, un module de reconnaissance de comportements erronés a été développé et ajouté au logiciel. Ce module a, en définitive, validé son applicabilité dans ce contexte.

Sommairement, nous avons réalisé tous les objectifs fixés à l'introduction. L'approche créée répond aux problèmes rencontrés avec la localisation par puces RFID passive comme, par exemple, les forces de signaux erronés ou bruités imprévisiblement par l'environnement.

\section{$5.2 \quad$ Revue du modèle développé}

En résumé, notre nouvelle approche novatrice de localisation d'étiquettes RFID passive consiste en un algorithme amélioré de positionnement par multilatération. Concrètement, l'algorithme proposé comporte trois étapes principales. Tout d'abord, lors de la première étape, on applique successivement les trois filtres suivants sur les forces de signaux : le filtre de réduction des fausses lectures, le filtre de réduction de crête et finalement, le filtre de pondération par moyenne gaussienne. Ensuite, la deuxième étape consiste au calcul de la position par multilatération elliptique. Plus précisément, la position est obtenue en transformant les RSSI en ellipses positionnées et orientées dans la direction de l'antenne correspondante pour, ensuite, calculer le barycentre de la zone d'intersection entre ces disques elliptiques. Finalement, la troisième étape consiste en un nouveau filtre de post-traitement effectué sur la position 
calculée par multilatération afin d'en extraire la position finale en y ajoutant des règles qui définissent le comportement de mouvements pour les rendre plus naturels, stable et précis.

Ensuite, nous avons implémenté et testé l'algorithme pour mesurer et comparer ses performances. Avec une précision de $11.64 \mathrm{~cm}$ sur une superficie de $6 \mathrm{~m}^{2}$, on peut conclure que notre système de localisation est plus précis que les systèmes comparables. De plus, l'expérimentation avec le système de reconnaissance de comportements erronés confirme que nous avons obtenu de très bons résultats et que le système de positionnement peut être utilisé efficacement pour la reconnaissance d'étapes d'une activité.

\subsection{Limitation et travaux futurs}

Certes, notre approche répond aux différents objectifs fixés à l'avance et permet d'effectuer une localisation d'objets mobile de manière suffisamment fiable et précise; mais, elle souffre tout de même de quelques limitations qui ont été mises suffisamment en évidence lors de la phase d'expérimentation pour être remarquées. Premièrement, l'utilisation d'un filtre de Kalman [56] est une bonne piste de solution pour améliorer le filtrage des forces de signaux en augmentant la réactivité comparativement au filtre de pondération par moyenne gaussienne. Deuxièmement, certains paramètres bénéficieraient grandement d'une calibration ou d'un ajustement automatique en temps réel. Pour ce faire, certaines pistes de solution devraient être explorées comme, par exemple, une estimation de l'imprécision de la position calculée ou l'estimation de la vitesse de déplacement d'un objet. Dans le cas où ces pistes de solution s'avèrent possibles, cela permettrait d'ajuster en temps réel la vitesse maximale utilisée par l'algorithme de filtrage des positions (comportement de mouvement) ou d'élaborer un tout nouveau filtre utilisant judicieusement ces nouvelles informations. Pour finir, il serait très intéressant d'aborder le problème de la localisation en trois dimensions. 


\subsection{Bilan personnel du travail de recherche}

Comme conclusion finale, je voudrais profiter de ces quelques derniers mots pour faire une brève évaluation personnelle de mon initiation au monde de la recherche. Bien évidemment, je me suis investi de manière constante avec persévérance et acharnement tout au long de ce travail. En plus, l'expérience et les connaissances acquises valaient bien tous ces efforts. La nature concrète et stimulante de cette recherche dans le cadre de l'infrastructure de l'habitat intelligent au laboratoire LIARA m'a permis de développer mes habiletés à la recherche scientifique. Comme cette première expérience s'est très bien déroulée, elle m'a encouragé à aller de l'avant et à entamer des études doctorales pour continuer à développer mes connaissances et mon expérience en lien avec mon domaine d'expertise. 


\section{BIBLIOGRAPHIE}

[1] U. N. (UN), "World Population Ageing 2009," Department of Economic and Social Affairs: Population Division, pp. 129, 2009.

[2] "Rapport de la consultation publique sur les conditions de vie des aînés," Ministère de la Famille et des Aînés du Québec, pp. 1-168, 2008.

[3] G. S. Abdulrazak B., Bouchard B., Pigot H., Mokhtari M. (Eds.), "Toward Useful Services for Elderly and People with Disabilities," Proc. of International Conference on Smart Homes and Health Telematics, ICOST 2011, Montreal, Springer LNCS, pp. 327, 2011.

[4] S. S. Intille, "Designing a Home of the Future," IEEE Pervas Comput, pp. 76-82, 2002.

[5] M. A. Pigot H., Giroux S., "The intelligent habitat and everyday life activity support," in 5th International Conference on Simulations in Biomedicine, 2003.

[6] B. J. Mihailidis A., Canido M., and Hoey J., "The Use of an Intelligent Prompting System for People with Dementia: A Case Study," Journal of ACM Interactions, pp. 34-37, 2007.

[7] T. L. S. Giroux, A. Bouzouane, B. Bouchard, H. Pigot, J. Bauchet, "The Praxis of Cognitive Assistance in Smart Homes, in: B. Gottfried, H.K. Aghajan (Eds.) Behaviour Monitoring and Interpretation," IOS Press, vol. BMI Book, pp. 183-211, 2009.

[8] C. Ramos, J.C. Augusto, and D. Shapiro, "Ambient Intelligence: the Next Step for Artificial Intelligence," IEEE Intelligent Systems, pp. 15-18, 2008.

[9] Bouchard K., Bouchard B., and B. A., "Guideline to Efficient Smart Home.Design for Rapid AI Prototyping: A Case Study," Proceedings of the 5th ACM International Conference on Pervasive Technologies Related to Assistive Environments (PETRA'12), Association for Computing Machinery (ACM) publisher, June 6-8, Crete Island, Greece, pp. 1-8, June 6-8, 2012.

[10] Augusto J.C., and N. C. D., "Designing Smart Homes: the role of artificial intelligence, State of the Art Survey," Lecture Notes in Artificial Intelligence (LNAI 4008), Springer-Verlag, pp. 1-183, 2006.

[11] B. Bouchard, A. Bouzouane, and S. Giroux, "A keyhole plan recognition model for alzheimer's patients," Journal of applied artificial intelligence, Taylor \& Francis, pp. 623-658, 2007.

[12] VanTassel M., J. Bouchard, Bouchard B. et al., "Guidelines for Increasing Prompt Efficiency in Smart Homes according to the Resident's Profile and Task Characteristics "Proceedings of the 9 th Int. Conference on Smart Homes and Health Telematics (ICOST), Springer publisher, Lecture Notes in Computer Science (LNCS), Montréal (QC), Canada, pp. 112-120, June 20-23, 2011.

[13] P. Roy, B. Bouchard, and A. Bouzouane, "Challenging issues of ambient activity recognition for cognitive assistance," Handbook of research on Ambient Intelligence and Smart Environnements: Trends and Perspective, M. a. N. C. Editors, pp. 320-345, 2011. 
[14] D. E. Riedel, S. Venkatesh, and W. Liu, "Spatial activity recognition in a smart home environment using a chemotatic model," Proceedings of the 2005 intelligent sensors, sensor networks and information processing conference, Melbourne, Australia, 2005.

[15] D. Fortin-Simard, and al., "Accurate passive RFID localization for smart homes," IEEE NESEA, 2012.

[16] J. Zhou, and J. Shi, "RFID localization algorithms and applications-a review," Journal of Intelligent Manufacturing, vol. 20, no. 6, pp. 695-707, 2008.

[17] M. Addlesee, R. Curwen, S. Hodges et al., "Implementing a Sentient Computing System," IEEE Computer, vol. 34, no. 8, pp. 50-56, 2001.

[18] J. Hoey, M. J. Chantler, E.Trucco et al., "Tracking using flock of features, with application to assisted handwashing," British Machine Vision Conference BMVC, pp. 367-376, 2006.

[19] Rocher P-O, Bouchard B., and B. A., "A New Platform to Easily Experiment Activity Recognition Systems Based on Passive RFID Tags: Experimentation with Data Mining Algorithms," International Journal of Smart Homes (IJSH), Science and Engineering Support Society (SERSC), vol. 6, pp. 7-24, april, 2012.

[20] C. Hekimian-Williams, B. Grant, and P. Kumar, "Accurate localization of RFID tags using phase difference," 2010 IEEE International Conference on RFID IEEE RFID 2010, pp. 89-96, 2010.

[21] Kelepouris T., Pramatari K., and D. G., "RFID-enabled traceability in the food supply chain," Journal of Industrial Management \& Data Systems, vol. 107 no. 2, pp. 183-200, 2007.

[22] Mehmet Erkan Yuksel, and A. S. Yuksel, "RFID Technology in Business Systems and Supply Chain Management," Journal of Economic and Social Studies, pp. 53-71, 2011.

[23] M. Yamin, "Crowd Management with RFID \& Wireless Technologies," IEEE international Conference on Networks and Communications, pp. 439-442, 2009.

[24] D. Hähnel, W. Burgard, D. Fox et al., "Mapping and Localization with RFID Tags." pp. 10151020.

[25] P. Vorst, S. Schneegans, Y. Bin et al., "Self-Localization with RFID snapshots in densely tagged environments." pp. 1353-1358.

[26] D. Joho, C. Plagemann, and W. Burgard, "Modeling RFID signal strength and tag detection for localization and mapping," in Proceedings of the 2009 IEEE international conference on Robotics and Automation, Kobe, Japan, 2009, pp. 1213-1218.

[27] K. Chawla, and G. Robins, "An RFID-based object localisation framework," International journal of radio frequency identification technology and applications vol. 3, pp. 2-30, 2011.

[28] Y. Zhang, M. G. Amin, and S. Kaushik, "Localization and tracking of passive RFID tags based on direction estimation," International Journal of Antennas and Propagation, vol. 2007, no. 17426, pp. 9, 2007.

[29] C. Y. Chen, J. P. Yang, G. J. Tseng et al., "An Indoor positioning technique based on fuzzy logic." pp. 854-857. 
[30] J. Curtin, R. Kauffman, and F. Riggins, "Making the 'MOST' out of RFID technology: a research agenda for the study of the adoption, usage and impact of RFID," Information Technology \& Management, vol. 8, no. 2, pp. 87-110, 2007.

[31] A. P. Sample, C. Macomber, J. Liang-Ting et al., "Optical localization of passive UHF RFID tags with integrated LEDs." pp. 116-123.

[32] Y. Lei, C. Jiannong, Z. Weiping et al., "A hybrid method for achieving high accuracy and efficiency in object tracking using passive RFID." pp. 109-115.

[33] L. M. Ni, Y. Liu, Y. C. Lau et al., "LANDMARC: Indoor Location Sensing Using Active RFID," Wireless Networks, vol. 10, pp. 701-710, 2004.

[34] Q. Fu, and R. Guenther, "Active RFID Trilateration and Location Fingerprinting Based on RSSI for Pedestrian Navigation," Journal of Navigation, vol. 62, pp. 323-340, 2009.

[35] K. Kim, and M. Kim, "RFID-based location-sensing system for safety management," Personal Ubiquitous Comput., vol. 16, no. 3, pp. 235-243, 2012.

[36] Z. Junyi, and S. Jing, RFID localization algorithms and applications-a review, Heidelberg, ALLEMAGNE: Springer, 2009.

[37] D. Lymberopoulos, A. Bamis, and A. Savvides, "Extracting spatiotemporal human activity patterns in assisted living using a home sensor network," in Proc. of the 1st int. conf. on Pervasive Technologies Related to Assisted Environments. ACM, Athens, Greece, 2008.

[38] K. P. Fishkin, B. Jiang, M. Philopose et al., "I Sense a Disturbance in the Force : Unobtrusive Detection of Interactions with RFID-tagged Objects," Intellectual Property, vol. 3205/ 2004, pp. 268-282, 2004.

[39] G.-y. Jin, X.-y. Lu, and M.-S. Park, "An Indoor Localization Mechanism Using Active RFID Tag," in Proceedings of IEEE International Conference on Sensor Networks, Ubiquitous, and Trustworthy Computing, Newport Beach, California, USA, 2006.

[40] S. N. Othman, "Node positioning in zigbee network using trilateration method based on the received signal strength indicator (RSSI)," European Journal of Scientific Research, vol. 46, no. 1, pp. 048-061, 2010.

[41] Kamin Whitehouse, Chris Karlof, and D. Culler, "A practical evaluation of radio signal strength for ranging-based localization," ACM SIGMOBILE Mobile Computing and Communications Review, vol. 11, no. 1, pp. 41-52, 2007.

[42] B.-S. Choi, and J.-J. Lee, "Mobile robot localization in indoor environment using RFID and sonar fusion system," in Proceedings of the 2009 IEEE, RSJ international conference on Intelligent robots and systems, St. Louis, MO, USA, 2009, pp. 2039-2044.

[43] C. Heesung, and H. Kyuseo, "Combination of RFID and Vision for Mobile Robot Localization," in Intelligent Sensors, Sensor Networks and Information Processing Conference, Melbourne, Australia, 2005, pp. 75-80.

[44] A. Milella, D. Di Paola, G. Cicirelli et al., "RFID tag bearing estimation for mobile robot localization." pp. 1-6. 
[45] A. Parr, R. Miesen, F. Kirsch et al., "A novel method for UHF RFID tag tracking based on acceleration data." pp. 110-115.

[46] A. Bekkali, H. Sanson, and M. Matsumoto, "RFID Indoor Positioning Based on Probabilistic RFID Map and Kalman Filtering " in in Third IEEE International Conference on Wireless and Mobile Computing, Networking and Comminications, New York, USA, 2007.

[47] J. L. Brchan, Z. Lianlin, W. Jiaqing et al., "A real-time RFID localization experiment using propagation models," in IEEE International Conference on RFID, Orlando, FL, USA, 2012, pp. 141-148.

[48] C. Wang, H. Wu, and N.-F. Tzeng, "RFID-Based 3-D Positioning Schemes," in 26th IEEE International Conference on Computer Comminications, Anchorage, Alaska, USA, 2007, pp. $1235-1243$.

[49] J. Han, Y. Zhao, Y. S. Cheng et al., "Improving Accuracy for 3D RFID Localization," International Journal of Distributed Sensor Networks, pp. 9, 2012.

[50] J. R. Smith, K. P. Fishkin, B. Jiang et al., "RFID-based techniques for human activity detection," Commun. ACM, vol. 48, pp. 39-44, 2005.

[51] D. Patterson, H. Kautz, and D. Fox, "Pervasive computing in the home and community," in Pervasive Computing in Healthcare, ed: CRC Press, pp. 79-103, 2007.

[52] K. Bouchard, B. Bouchard, and A. Bouzouane, "Qualitative spatial activity recognition using a complete plateform based on passive RFID tags: Experimentations and results," in Proc. of the ICOST. Springer Berlin / Heidelberg, Montreal, Canada, 2011.

[53] Y. Zhang, M. G. Amin, and S. Kaushik, "Localization and Tracking of Passive RFID Tags Based on Direction Estimation," International Journal of Antennas and Propagation, vol. 2007, pp. 1-9, 2007.

[54] J. Brusey, C. Floerkemeier, M. Fletcher et al., "Reasoning about Uncertainty in Location Identification with RFID," Workshop on Reasoning with Uncertainty in Robotics at IJCAI- 2003, pp. 23-30, 2003.

[55] C. W. Reynolds, "Steering Behaviors for Autonomous Characters," Proc. Game Developers Conference, pp. 763-782, 1999.

[56] M. S. Grewal, and A. P. Andrews, "Linear optimal filters and predictors," Kalman Filtering: Theory and Practice Using MATLAB®, Third Edition, pp. 131-181, 2008. 